University of Tennessee Health Science Center

UTHSC Digital Commons

\title{
Challenging the Paradigm of Clinical Triazole Resistance in Aspergillus fumigatus
}

Jeffrey Michael Rybak

University of Tennessee Health Science Center

Follow this and additional works at: https://dc.uthsc.edu/dissertations

Part of the Medicine and Health Sciences Commons, and the Microbiology Commons

\section{Recommended Citation}

Rybak, Jeffrey Michael (0000-0002-9317-0935), "Challenging the Paradigm of Clinical Triazole Resistance in Aspergillus fumigatus" (2019). Theses and Dissertations (ETD). Paper 494. http://dx.doi.org/10.21007/ etd.cghs.2019.0487.

This Dissertation is brought to you for free and open access by the College of Graduate Health Sciences at UTHSC Digital Commons. It has been accepted for inclusion in Theses and Dissertations (ETD) by an authorized administrator of UTHSC Digital Commons. For more information, please contact jwelch30@uthsc.edu. 


\title{
Challenging the Paradigm of Clinical Triazole Resistance in Aspergillus fumigatus
}

\begin{abstract}
Invasive aspergillosis is a leading cause of morbidity and mortality among immunocompromised populations and is predicted to cause more than 200,000 life- threatening infections each year. Aspergillus fumigatus is the most prevalent pathogen isolated from patients with invasive aspergillosis, accounting for more than $60 \%$ of all cases. Currently, the only antifungal agents available with consistent activity against $A$. fumigatus are the mold-active triazoles and amphotericin $B$, of which the triazoles commonly represent both front-line and salvage therapeutic options. Unfortunately, the treatment of infections caused by A. fumigatus has recently been further complicated by the global emergence of triazole resistance among both clinical and environmental isolates, and a large proportion of this resistance remains unexplained. In this work, we characterize the contributions of previously identified mechanisms of triazole resistance, including mutations in the sterol-demethylase- encoding gene cyp51A, overexpression of sterol-demethylase genes, and overexpression of the efflux pump-encoding gene abcC, among a large collection of highly triazole- resistant clinical A. fumigatus isolates. Upon revealing that these mechanisms alone cannot substantiate the majority of triazole resistance exhibited by this collection, we then characterize the direct contribution of two additional efflux pump-encoding genes, abcA and atrl. Increased expression of abcA and atrl has previously been associated with triazole resistance in clinical isolates of $A$. fumigatus, and both of these genes exhibit a high degree of homology with the well characterized Candida albicans triazole efflux pump-encoding gene, CDR1. However, deletion of either abcA or atrl in triazole-resistant clinical isolates which overexpress these genes, did not result in a significant change in triazole susceptibility. Finally, upon demonstrating that the canonical mechanisms of triazole resistance poorly explain the high level of triazole resistance observed in this collection of clinical isolates, we subsequently describe the identification and characterization of a novel genetic determinant of triazole resistance. Mutations in the HMG-CoA reductase encoding gene, hmg1, were identified in a majority of triazole-resistant clinical isolates in our collection. Introduction of three different hmg1 mutations, predicted to encode residue alterations in the conserved sterol sensing domain of $\mathrm{Hmg} 1$, resulted in significantly increased resistance to the triazole class of agents. Additionally, correction of an hmg1 mutation in a pan-triazole-resistant clinical isolate of A. fumigatus with a novel Cas9-ribonucleoprotein (RNP) mediated system, was shown to restore clinical susceptibility to all triazole agents. Mutations in hmg1 were also shown to lead to the accumulation of ergosterol precursors, such as eburicol, by sterol profiling, while not altering the expression of sterol-demethylase genes. Taken together, the findings described in this work serve to demonstrate that mutations in hmg1 are a common and significant genetic determinant of triazole resistance in clinical isolates of A. fumigatus."
\end{abstract}

Document Type

Dissertation

Degree Name

Doctor of Philosophy (PhD)

Program

Biomedical Sciences

Research Advisor

P. David Rogers, PharmD, PhD

Keywords

Aspergillus, resistance, triazole 


\section{Subject Categories}

Medicine and Health Sciences | Microbiology 
Challenging the Paradigm of Clinical Triazole Resistance in Aspergillus fumigatus

\author{
A Dissertation \\ Presented for \\ The Graduate Studies Council \\ The University of Tennessee \\ Health Science Center
}

\author{
In Partial Fulfillment \\ Of the Requirements for the Degree \\ Doctor of Philosophy \\ From The University of Tennessee
}

By

Jeffrey Michael Rybak

May 2019 
Chapter 1 (C) 2018 by Oxford University Press Chapters 2, 3, and 4 (C) 2019 by American Society for Microbiology All other material (c) 2019 by Jeffrey Michael Rybak.

All rights reserved. 


\section{DEDICATION}

To my parents Michael and Stephanie, my brother Matt, and my Aunt Carol for all their love and support. To my Aunt Cindy, Uncle Mike, Grandfather Alois, and Grandfather "Hub", you are greatly missed. 


\section{ACKNOWLEDGEMENTS}

First, I would like to express my sincere gratitude to my advisor, Dave Rogers. I could not have asked for a more supportive and encouraging mentor. Thank you for all you have taught me, the countless opportunities you have provided, and for the clear investment you made in my development as an academic pharmacist and as a scientist

Second, I would like to thank my committee members, Jarrod Fortwendel, Glen Palmer, Brian Peters, Ramin Homayouni, and Elizabeth Fitzpatrick. I greatly appreciate each of your insight, guidance, and support.

Third, I would like to thank all the members of the Rogers Laboratory past and present, Qing Zhang, Kathy Barker, Arielle Butts, Beth Berkow, Sarah Whaley, Andy Nishimoto, Laura Doorley, Rachel Stein, Yu Li, and Cheshta Sharma, for their friendship, encouragement, and patience. I would also like to give a special thanks to Jarrod Fortwendel and the members of the Fortwendel laboratory, Qusai Al Abdallah, Adela Martin-Vicente, Ana Camila Oliveira Souza, Ashley Nywening, and Wenbo Ge for welcoming me as an unofficial member of the lab. Moreover, I thank all the members of UTHSC Fungal Pathogens group, for their friendship and the wonderful learning and working environment they have each helped to create.

Finally, I am eternally grateful to all my family and friends for their continued support in the pursuit of my professional and personal goals.

The work described herein was supported by the American College of Clinical Pharmacy Research Institute Futures Grant Award (JMR) and National Institute of Allergy and Infectious Diseases (NIAID) grants R01 A1058145 (PDR) and R01 AI106925 (JRF). 


\begin{abstract}
Invasive aspergillosis is a leading cause of morbidity and mortality among immunocompromised populations and is predicted to cause more than 200,000 lifethreatening infections each year. Aspergillus fumigatus is the most prevalent pathogen isolated from patients with invasive aspergillosis, accounting for more than $60 \%$ of all cases. Currently, the only antifungal agents available with consistent activity against $A$. fumigatus are the mold-active triazoles and amphotericin $\mathrm{B}$, of which the triazoles commonly represent both front-line and salvage therapeutic options. Unfortunately, the treatment of infections caused by A. fumigatus has recently been further complicated by the global emergence of triazole resistance among both clinical and environmental isolates, and a large proportion of this resistance remains unexplained.

In this work, we characterize the contributions of previously identified mechanisms of triazole resistance, including mutations in the sterol-demethylaseencoding gene cyp $51 \mathrm{~A}$, overexpression of sterol-demethylase genes, and overexpression of the efflux pump-encoding gene $a b c C$, among a large collection of highly triazoleresistant clinical $A$. fumigatus isolates. Upon revealing that these mechanisms alone cannot substantiate the majority of triazole resistance exhibited by this collection, we then characterize the direct contribution of two additional efflux pump-encoding genes, $a b c A$ and $a t r I$. Increased expression of $a b c A$ and $a t r I$ has previously been associated with triazole resistance in clinical isolates of $A$. fumigatus, and both of these genes exhibit a high degree of homology with the well characterized Candida albicans triazole efflux pump-encoding gene, $C D R 1$. However, deletion of either $a b c A$ or $a t r I$ in triazole-resistant clinical isolates which overexpress these genes, did not result in a significant change in triazole susceptibility.

Finally, upon demonstrating that the canonical mechanisms of triazole resistance poorly explain the high level of triazole resistance observed in this collection of clinical isolates, we subsequently describe the identification and characterization of a novel genetic determinant of triazole resistance. Mutations in the HMG-CoA reductase encoding gene, $h m g 1$, were identified in a majority of triazole-resistant clinical isolates in our collection. Introduction of three different $h m g l$ mutations, predicted to encode residue alterations in the conserved sterol sensing domain of Hmgl, resulted in significantly increased resistance to the triazole class of agents. Additionally, correction of an hmgl mutation in a pan-triazole-resistant clinical isolate of A. fumigatus with a novel Cas9-ribonucleoprotein (RNP) mediated system, was shown to restore clinical susceptibility to all triazole agents. Mutations in $\mathrm{hmgl}$ were also shown to lead to the accumulation of ergosterol precursors, such as eburicol, by sterol profiling, while not altering the expression of sterol-demethylase genes. Taken together, the findings described in this work serve to demonstrate that mutations in hmgl are a common and significant genetic determinant of triazole resistance in clinical isolates of A. fumigatus.
\end{abstract}




\section{TABLE OF CONTENTS}

\section{CHAPTER 1. THE EMERGING THREAT OF TRIAZOLE-RESISTANT

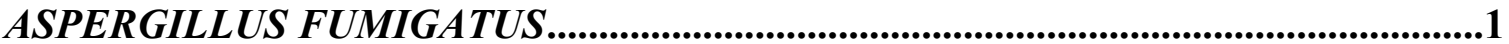

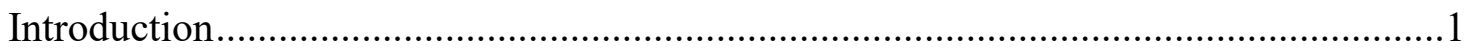

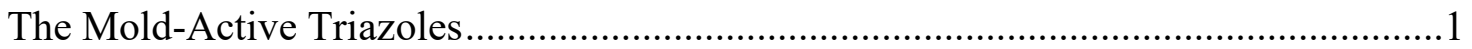

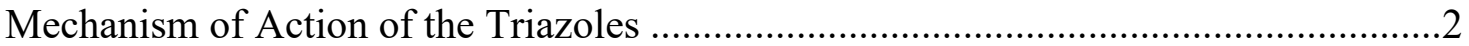

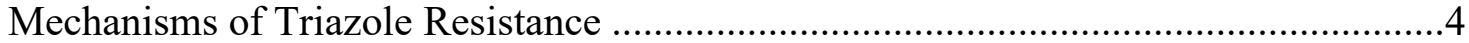

Mutations in the A. fumigatus Sterol-Demethylase Gene cyp $51 A$............................4

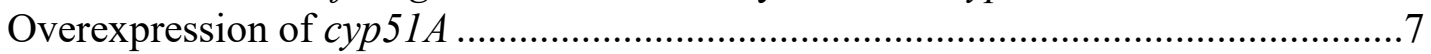

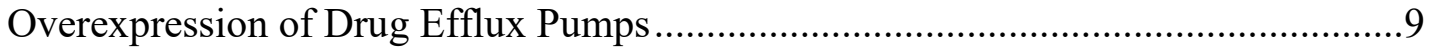

Emerging Mechanisms of Triazole Resistance ................................................... 10

Epidemiology of Triazole Resistance ..................................................................... 10

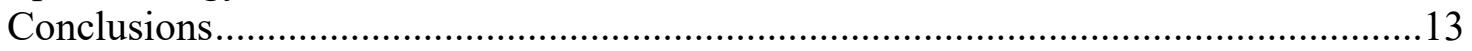

\section{CHAPTER 2. CHALLENGING THE PARADIGM OF CLINICAL TRIAZOLE}

RESISTANCE IN ASPERGILLUS FUMIGATUS...............................................14

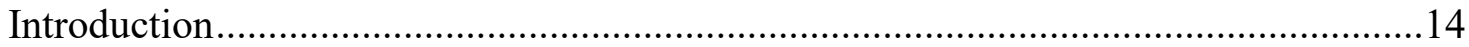

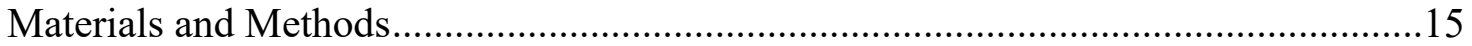

Isolates, Media, and Growth Conditions Used .................................................15

Whole Genome Sequencing ...................................................................... 15

Construction of Promoter Replacement Repair Templates and Cas9-RNP for

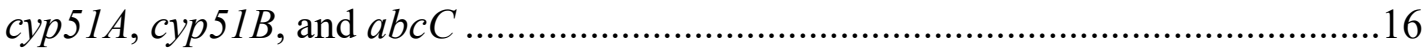

Assessment cyp 51A, cyp 51B, and abcC Expression by RTqPCR ...........................16

Construction of Allele Replacement Repair Templates and Cas9-RNP for the

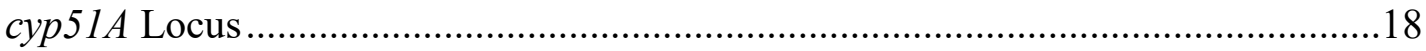

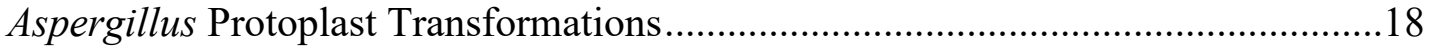

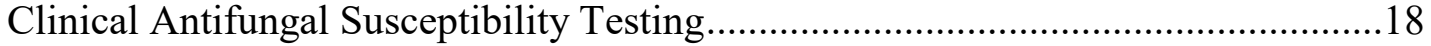

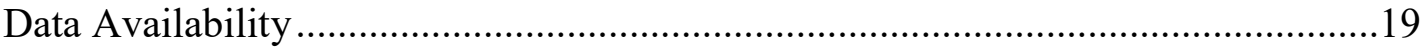

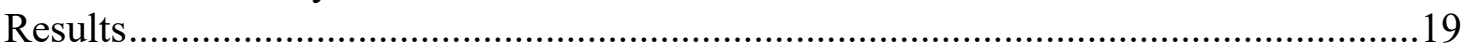

Mutations in Sterol-Demethylase Alone Poorly Explain Clinical Triazole

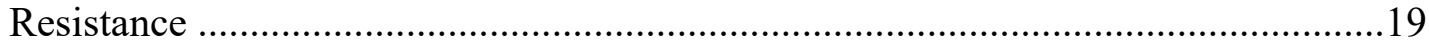

Analysis of the Expression of the Sterol-Demethylases Genes cyp51A and cyp51B Among Triazole-Resistant Clinical Isolates. .........................................21

Constitutive Overexpression of cyp51B Decreases Triazole Susceptibility ..............22

Analysis of the Expression of the Efflux Pump-Encoding Gene abcC Among

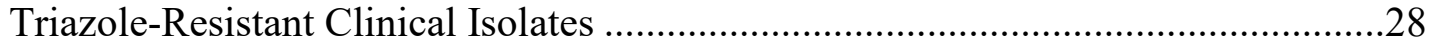

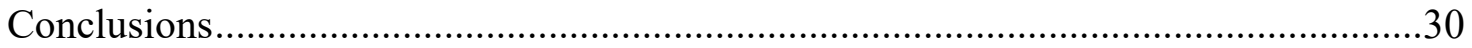

\section{CHAPTER 3. THE ASPERGILLUS FUMIGATUS EFFLUX PUMP- ENCODING GENES $A B C A$ AND $A T R I$ ARE NOT SIGNIFICANT} CONTRIBUTORS TO CLINICAL TRIAZOLE RESISTANCE............................31

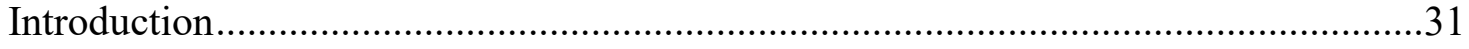

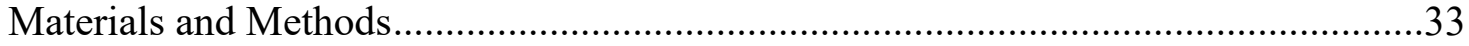

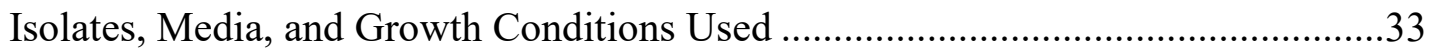


Transcriptional Profiling .........................................................................................33

Construction of Efflux Pump-Encoding Gene Deletion Repair Templates .................33

Aspergillus Protoplast Transformations..................................................................

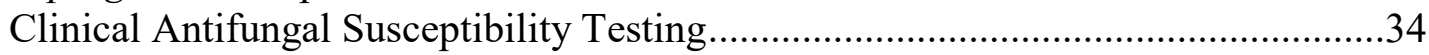

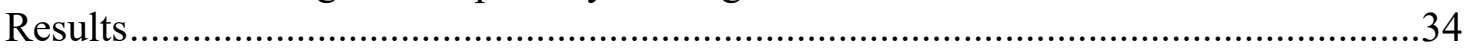

Analysis of the Expression of the Efflux Pump-Encoding Genes abcA and atrI

Among Triazole-Resistant Clinical Isolates. ............................................................34

Deletion of the Efflux Pump-Encoding Genes $a b c A$ and atrI Has Minimal

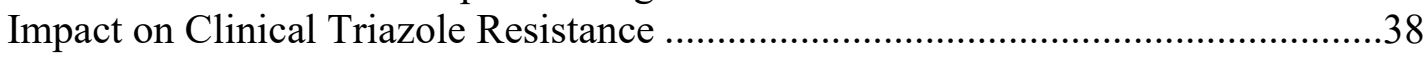

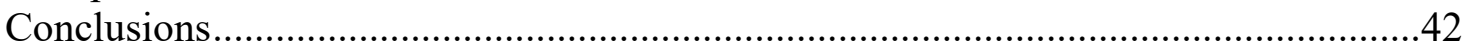

\section{CHAPTER 4. MUTATIONS IN THE ASPERGILLUS FUMIGATUS HMG-} COA REDUCTASE GENE, HMG1, A NOVEL GENETIC DETERMINANT OF CLINICAL TRIAZOLE RESISTANCE ........................................................44

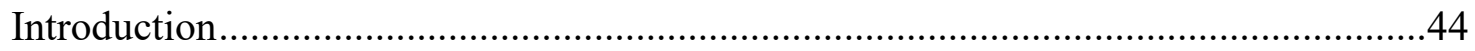

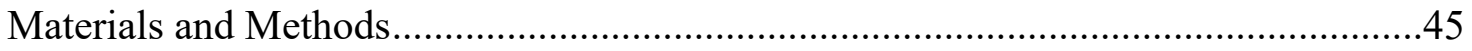

Isolates, Media, and Growth Conditions Used .......................................................45

Construction of Allele Replacement Repair Templates and Cas9-RNP for the hmgl Locus .................................................................................................45

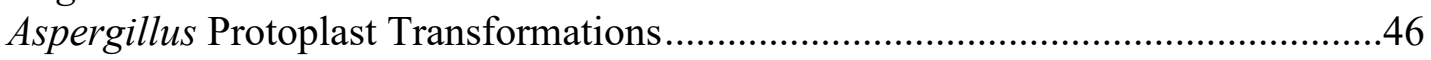

Clinical Antifungal Susceptibility Testing..............................................................46

Comprehensive Sterol Profiling............................................................................4

Assessment cyp51A and cyp51B Expression by RTqPCR ……….........................48

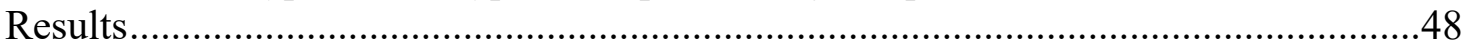

Mutations in hmgl Are Common Among Triazole-Resistant Clinical Isolates of

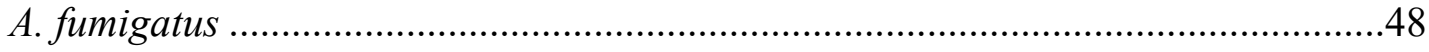

Mutations in hmgl Confer Resistance to the Triazole Class of Antifungals .............49

Mutations in hmgl Lead to Accumulation of Ergosterol Precursors..........................53

Mutations in hmgl Do Not Lead to Increased Expression of Sterol-Demethylase

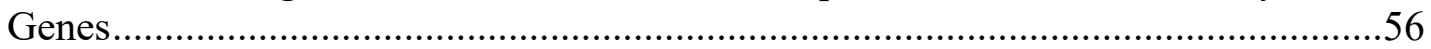

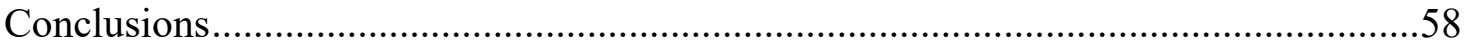

CHAPTER 5. DISCUSSION ..........................................................................................59

LIST OF REFERENCES .............................................................................................63

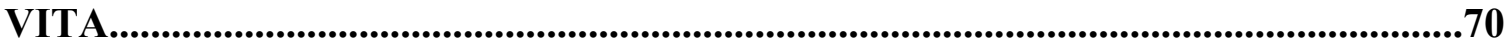




\section{LIST OF TABLES}

Table 1-1. cyp51A mutations by residue and relative impact on triazole MIC ..............6

Table 2-1. Oligonucleotides used for cyp51A, cyp51B, and $a b c C$ experiments ...........17

Table 2-2. Triazole MIC of clinical isolates and derivative $c y p 51 A^{\mathrm{WT}}$ strains ............20

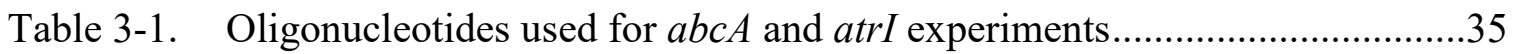

Table 3-2. Relative expression of efflux pump-coding genes of interest among

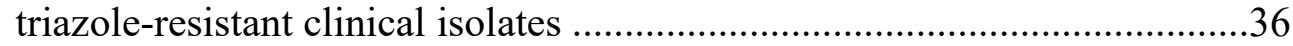

Table 3-3. Triazole MIC of clinical isolates and derivative efflux pump-encoding gene deletion strains .............................................................................4

Table 4-1. Oligonucleotides used for $h m g 1$, cyp 51A, and cyp 51B experiments ...........47

Table 4-2. Mutations in genes involved in ergosterol biosynthesis which are unique to triazole resistant isolates 


\section{LIST OF FIGURES}

Figure 1-1. Structures of the clinically relevant triazoles .............................................

Figure 1-2. Canonical mechanisms of triazole resistance in A. fumigatus .......................3

Figure 1-3. cyp51A mutations associated with triazole resistance ................................5

Figure 1-4. Regulation of $c y p 51 A$ expression....................................................

Figure 2-1. Relative expression of cyp $51 A$ and cyp $51 B$ among triazole-resistant clinical isolates

Figure 2-2. Schematic of Cas9-RNP mediated promoter replacement system .26

Figure 2-3. Relative expression of cyp51A and cyp51B, and voriconazole MIC in promoter replacement strains $a k u B^{\mathrm{KU} 80} P_{h s p A}$ cyp $51 A$ and $a k u B^{\mathrm{KU} 80} P_{h s p A}$ cyp $51 B$

Figure 2-4. Relative expression of $a b c C$ in triazole-resistant clinical isolates and the constitutive overexpression strain $a k u B^{\mathrm{KU} 80} P_{h s p A} a b c C$

Figure 3-1. Relative expression of $a b c A$ and $a t r I$ among triazole-resistant clinical isolates.

Figure 4-1. Mutations in $\mathrm{hmgl}$ observed in this collection of triazole-resistant clinical $A$. fumigatus isolates

Figure 4-2. Schematic of Cas9 ribonucleoprotein (RNP) editing technique incorporating a split hygromycin B resistance marker for creation of hmgl mutant strains

Figure 4-3. Impact of hmgl mutations on antifungal susceptibility.

Figure 4-4. Relative distribution of sterols and total ergosterol content of $\mathrm{hmgl}$ mutant strains

Figure 4-5. Expression of sterol-demethylase genes is not elevated among mutant hmgl strains. 


\section{CHAPTER 1. THE EMERGING THREAT OF TRIAZOLE-RESISTANT ASPERGILLUS FUMIGATUS*}

\section{Introduction}

Invasive aspergillosis is a leading cause of morbidity and mortality among immunocompromised patient populations, such as patients receiving solid organ or stem cell transplants, patients with hematologic malignancies, and those taking immunosuppressive agents.[1] Aspergillus, the causative agent of invasive aspergillosis, is a genus of septate, saprophytic mold containing hundreds of identified species. Of these only Aspergillus fumigatus, Aspergillus flavus, Aspergillus terreus, and Aspergillus niger commonly cause disease in humans, with A. fumigatus representing more than $60 \%$ of all clinical isolates.[2] Worldwide, invasive aspergillosis is conservatively estimated to cause more than 200,000 life threatening infections each year.[1,2] Mortality rates range considerably, from 30-90\%, varying by patient population as well as severity and duration of immunosuppression. [3-5] Unfortunately, the treatment of infections caused by $A$. fumigatus has recently been further complicated by the global emergence of triazole resistance among both clinical and environmental isolates.[6-9]

\section{The Mold-Active Triazoles}

Since the widespread introduction of itraconazole to clinical practice in 1992, the mold-active triazole antifungals have been utilized as both front-line and salvage therapeutic agents for the treatment of invasive aspergillosis. Voriconazole is now considered the therapy of choice, and is available in both intravenous and oral formulations.[10] Multiple studies comparing the efficacy of voriconazole to that of the previous standard of care, amphotericin B, have found voriconazole to be more efficacious and generally safer than either conventional (deoxycholate) or lipid formulations of amphotericin B.[11, 12] Isavuconazole was recently approved for the treatment of invasive aspergillosis, after demonstrating non-inferiority to the standard of care, voriconazole.[4, 10] Isavuconazole, as the prodrug isavuconazonium sulfate, is available in both intravenous and oral formulations.[13] Posaconazole, often used as prophylaxis against invasive fungal infections in high-risk patients such as those with acute myeloid leukemia, is also employed as second-line or salvage therapy for invasive aspergillosis, and is now available in both intravenous and delayed-release tablet formulations. Itraconazole is still used commonly worldwide, but its use in invasive aspergillosis has decreased due to both lower anti-aspergillus potency and the withdrawal of intravenous formulations from some markets.[10] While all these agents possess a

* Reprinted from final submission with permission. Rybak JM, Fortwendel JR, and Rogers PD. Emerging threat of triazole-resistant Aspergillus fumigatus. J Antimicrob Chemother. 2019 Apr 1;74(4):835-842. doi: 10.1093/jac/dky517 By permission of Oxford University Press. 
triazole active moiety (from which this class of agents gained its name), they can be grouped into two distinct pharmacophores (Figure 1-1). The compact triazoles such as voriconazole and isavuconazole consist of a triazole ring linked to aromatic groups such as difluorobenzine via a four-carbon backbone. While the extended side-chain triazoles, including itraconazole and posaconazole, possess both a halogenated phenyl ring as well as a long lipophilic side-chain comprised of five and six membered aromatic rings terminating in a triazolone group. The structural distinctions between these agents are thought to contribute to differences in stabilizing interactions sterol-demethylase enzymes.

\section{Mechanism of Action of the Triazoles}

In fungi, ergosterol is a key membrane lipid similar to mammalian cholesterol. Under normal growth conditions, ergosterol is plentiful in the fungal cell membrane and contributes to membrane integrity and fluidity, as well the appropriate positioning of fungal proteins within the membrane. The triazoles exert antifungal activity by blocking a key enzymatic step in the biosynthesis of ergosterol. Through direct interaction between $\mathrm{N} 4$ of the triazole ring and an iron atom present in the catalytic heme group, the triazoles competitively inhibit sterol-demethylase enzymes (Figure 1-2). Inhibition of steroldemethylase, such as Cyp51A and Cyp51B in the case of A. fumigatus, leads to the diversion of ergosterol precursors from the normal biosynthetic pathway and accumulation of alternative sterols including toxic $14 \alpha$-methylated sterols. It is believed that this depletion of ergosterol and buildup of $14 \alpha$-methylated sterols ultimately leads to inhibition of fungal growth.[14]

A. fumigatus possesses two genes which encode homologous but distinct steroldemethylase enzymes, cyp $51 \mathrm{~A}$ and cyp $51 \mathrm{~B}$. These genes share approximately $60 \%$ sequence identity, however the triazole antifungals have been observed to have significantly lower binding affinity for the Cyp51A enzyme.[15, 16] This reduced binding affinity is due in large part to the threonine amino acid present at position 301 (T301) of Cyp51A, which replaces the more common isoleucine residue present at this position in other fungal sterol-demethylase enzymes (including $A$. fumigatus Cyp51B).[17] The intrinsic fluconazole resistance of A. fumigatus appears to be a result of this single residue substitution. Surveillance studies of the potency of the mold-active triazoles against $A$. fumigatus clinical isolates have found isavuconazole, itraconazole, posaconazole, and voriconazole to all possess clinically relevant activity against $A$. fumigatus.[18] In general, the MIC of isavuconazole and itraconazole are higher, with $\mathrm{MIC}_{50}$ and $\mathrm{MIC}_{90}$ of 1 and $2 \mathrm{mg} / \mathrm{L}$, and 1 and $1 \mathrm{mg} / \mathrm{L}$, respectively (range $0.128-8 \mathrm{mg} / \mathrm{L}$ : isavuconazole, $0.25-4 \mathrm{mg} / \mathrm{L}$ : itraconazole). While the $\mathrm{MIC}_{50}$ and $\mathrm{MIC}_{90}$ for posaconazole and voriconazole are typically lower, 0.25 and $0.5 \mathrm{mg} / \mathrm{L}$ for both agents (range 0.06 $1 \mathrm{mg} / \mathrm{L}$ : posaconazole, $0.125-2 \mathrm{mg} / \mathrm{L}$ : voriconazole).[18]

The antifungal activity of the triazoles is concentration dependent. This activity has been shown to be best predicted by the free AUC to MIC ratio (fAUC:MIC).[19, 20] 


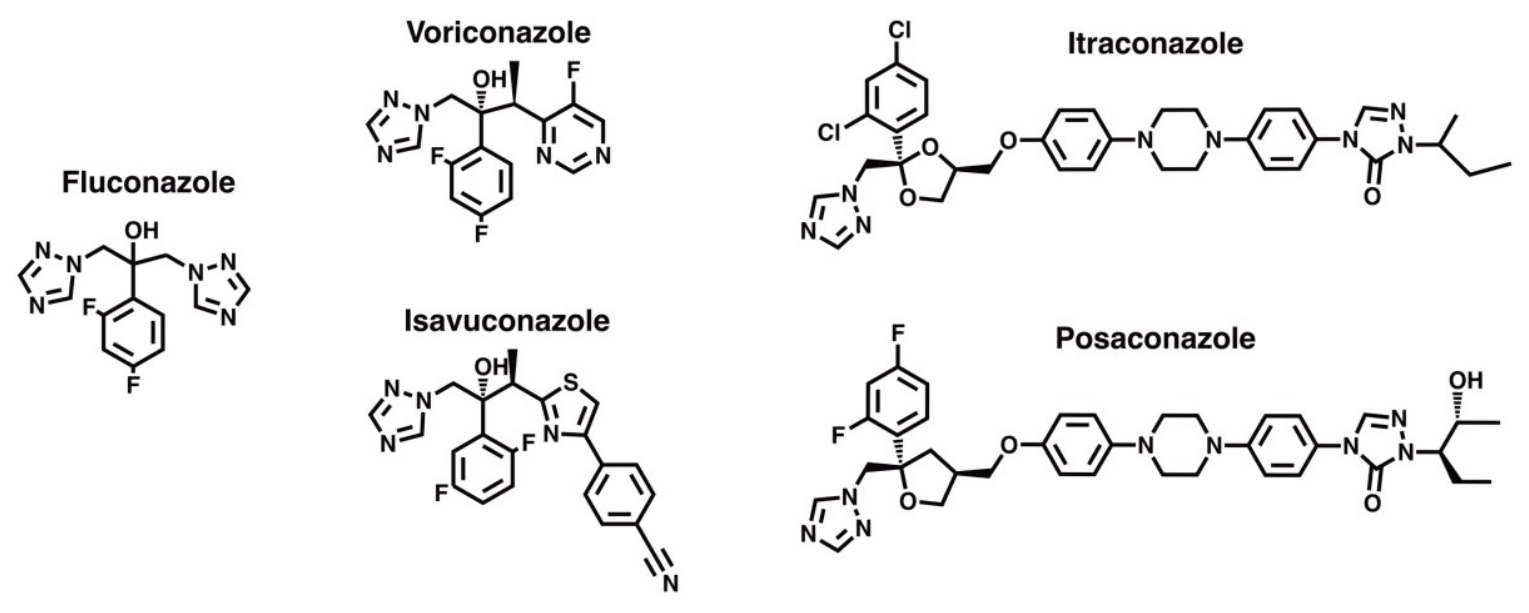

Figure 1-1. Structures of the clinically relevant triazoles.

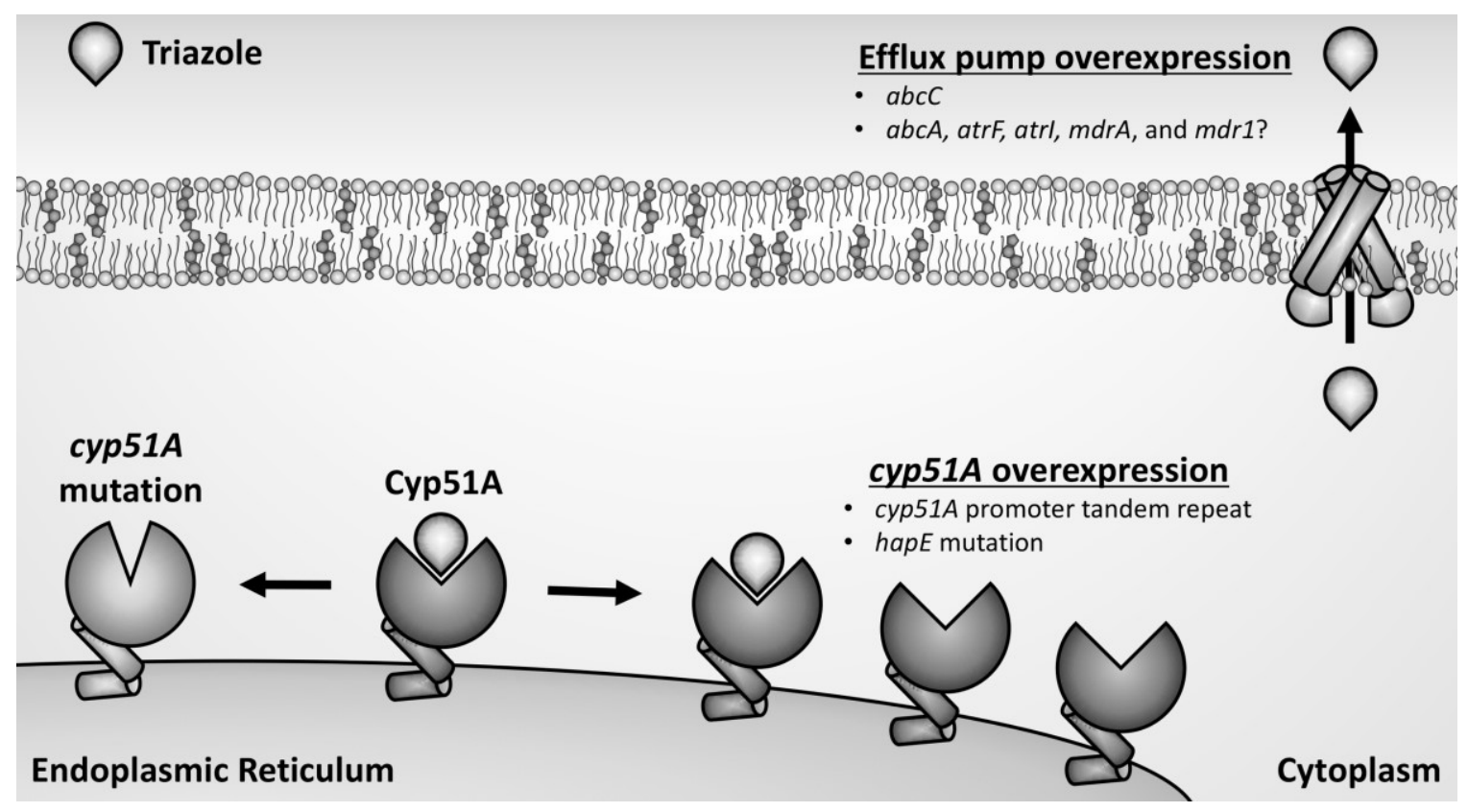

Figure 1-2. Canonical mechanisms of triazole resistance in A. fumigatus. 
In neutropenic murine models of pulmonary aspergillosis, the $f$ AUC:MIC needed to produce a $90 \%$ reduction in fungal burden (1-log reduction) was found to be roughly twice that of stasis for both posaconazole and isavuconazole. This was true for triazolesusceptible and triazole-resistant isolates.[19, 20] Additionally, the triazoles possess fungicidal activity at physiologically relevant concentrations against most triazolesusceptible $A$. fumigatus. [21] However, this fungicidal activity is also concentration dependent and varies from isolate to isolate.[22] Unlike amphotericin B, the mold-active triazoles do not possess a clinically relevant post-antifungal effect.[23]

\section{Mechanisms of Triazole Resistance}

Considerable research has led to the identification of multiple mechanisms of triazole resistance among other pathogenic fungi.[24] The most predominant mechanisms responsible for clinically encountered triazole resistance can be grouped into three distinct categories; 1) mutations in the sterol-demethylase gene, 2) overexpression of the sterol demethylase gene, and 3) overexpression of either ATP-Binding Cassette (ABC) or Major Facilitator Superfamily (MFS) type drug efflux pumps.[24] While relatively less is understood of the mechanisms of clinical triazole resistance among A. fumigatus, much of what is known focuses on these three paths to triazole resistance (Figure 1-2). [9, 25, 26]

\section{Mutations in the A. fumigatus Sterol-Demethylase Gene cyp51A}

The most commonly identified mechanism of triazole resistance among clinical isolates of A. fumigatus involves mutations in the sterol-demethylase gene, cyp51A.[8, 27] A great number of these mutations have been identified among both clinical and environmental isolates originating from across the globe, many of which are uniquely found in triazole resistant isolates (Figure 1-3). [8, 28, 29] A smaller subset of these mutations have been shown to directly contribute to triazole resistance through genetic manipulations in the laboratory setting (Table 1-1).[28, 30-36] Conversely, no mutations which are associated with clinical triazole resistance have been found in the cyp $51 B$ sterol-demethylase gene at this time.

Mutations in cyp51A associated with triazole resistance are believed to result in amino acid substitutions which either diminish the binding affinity of triazoles with the sterol-demethylase enzyme, or alter the enzyme structure in a manner which favors native substrate conversion and thus ergosterol biosynthesis.[28, 30-36] The majority of the known resistance associated mutations can be grouped into distinct regions of the steroldemethylase enzyme. The most common and best understood of which, encode amino acid substitutions occurring along the ligand access channel (residues G54, P216, F219, and M220) or in residues which are located near the catalytic site (Y121, G138).[15] The ligand access channel associated residue substitutions have been shown to greatly

decrease $A$. fumigatus susceptibility to both itraconazole and posaconazole, while having relatively less impact on voriconazole and isavuconazole susceptibilities.[30, 31, 36] It is 


\begin{tabular}{|c|c|}
\hline \multicolumn{2}{|c|}{$\begin{array}{c}\text { Tandem Repeat Associated } \\
\text { cyp51A Mutations }\end{array}$} \\
\hline \multicolumn{2}{|c|}{$\mathrm{TR}_{34} / \mathrm{L} 98 \mathrm{H}$} \\
\hline \multicolumn{2}{|c|}{$\mathrm{TR}_{46} / \underline{\mathrm{Y} 121 \mathrm{~F}} / \mathrm{T} 289 \mathrm{~A}$} \\
\hline \multicolumn{2}{|c|}{$\mathrm{TR}_{53}$} \\
\hline \multicolumn{2}{|c|}{$\mathrm{TR}_{53} / \mathrm{L} 98 \mathrm{H}$} \\
\hline \multicolumn{2}{|c|}{ Individual cyp51A Mutations } \\
\hline $\begin{array}{l}\text { Wild Type } \\
\text { Residue }\end{array}$ & $\begin{array}{l}\text { Amino Acid } \\
\text { Substitution }\end{array}$ \\
\hline $\mathrm{N} 22$ & $\mathrm{D}$ \\
\hline G54 & $\mathrm{E}, \mathrm{K}, \mathrm{R}, \mathrm{V}, \mathrm{W}$ \\
\hline G138 & $\mathrm{C}, \mathrm{R}, \mathrm{S}$ \\
\hline P216 & $\mathrm{L}$ \\
\hline F219 & $\mathrm{C}, \mathrm{I}, \mathrm{L}, \mathrm{S}$ \\
\hline M220 & $\mathrm{K}, \mathrm{I}, \mathrm{R}, \mathrm{T}, \mathrm{V}, \mathrm{W}$ \\
\hline A284 & $\mathrm{T}$ \\
\hline$\underline{\mathrm{I} 367}$ & $\mathrm{~F}$ \\
\hline Y431 & $\mathrm{C}, \mathrm{S}$ \\
\hline G432 & $\mathrm{A}, \mathrm{C}, \mathrm{S}$ \\
\hline G434 & $\mathrm{C}$ \\
\hline T440 & $\mathrm{A}$ \\
\hline G448 & $\mathrm{S}$ \\
\hline F495 & $\mathrm{L}$ \\
\hline Y491 & $\mathrm{H}$ \\
\hline
\end{tabular}

Figure 1-3. cyp51A mutations associated with triazole resistance.

Residues shown in bold are located along the ligand access channels, residues shown underlined directly interact with voriconazole. 
Table 1-1. cyp51A mutations by residue and relative impact on triazole MIC.

\begin{tabular}{|c|c|c|c|c|c|c|}
\hline \multirow[b]{2}{*}{ Triazole* } & \multicolumn{4}{|c|}{ Ligand Access Channel Residues } & \multicolumn{2}{|c|}{ Tandem Repeat Associated Residues } \\
\hline & G54 & P216 & F219 & M220 & $\mathrm{TR}_{34} / \mathrm{L} 98 \mathrm{H}$ & $\mathrm{TR}_{46} / \mathrm{Y} 121 \mathrm{~F} / \mathrm{T} 289 \mathrm{~A}$ \\
\hline Voriconazole & - & - & - & + & + & ++ \\
\hline Posaconazole & ++ & + & + & + & + & + \\
\hline Itraconazole & ++ & ++ & ++ & ++ & ++ & + \\
\hline
\end{tabular}

*data for isavuconazole not currently available, -; minimal to no impact on MIC, +; increase in MIC, ++; large increase in MIC 
believed that this agent specific triazole resistance is a consequence of these amino acid substitutions interfering with stabilizing interactions between the long hydrophobic side chains present in itraconazole and posaconazole, but absent in the other two agents. Conversely, a mutation conferring a tyrosine to phenylalanine substitution at position 121 (Y121F) has been shown to greatly decrease voriconazole susceptibility while having minimal impact on itraconazole or posaconazole.[32, 34] In x-ray crystallography experiments voriconazole has been observed to directly interact with tyrosine 121, though it is unknown if any of the other triazoles also interact with this residue.[15]

In addition to mutations in the open reading frame of the cyp51A gene, sequence duplications forming a tandem repeat in the promoter region of cyp5 $1 \mathrm{~A}$ have also been observed.[37] These tandem repeats do not alter the Cyp51A enzyme itself, but significantly increase to level of cyp $51 \mathrm{~A}$ gene expression, and in turn the amount of Cyp51A enzyme which must be inhibited by the triazoles to block ergosterol biosynthesis.[26] The impact of these tandem repeat associated mutations on triazole resistance will be further detailed in the subsequent section.

\section{Overexpression of $\operatorname{cyp} 51 \mathrm{~A}$}

The expression of cyp51A and cyp51B is regulated by a complex system of interacting transcription factors controlled by environmental conditions such as nutrient availability and stress. While differences are known to exist in the regulation of expression of the two sterol-demethylase genes, the majority of research has focused on cyp51A, which when overexpressed is known to contribute to clinical triazole resistance in $A$. fumigatus. $[9,26,34,35]$ It remains unknown if overexpression of cyp $51 B$ may also contribute to clinical resistance, but triazole-resistant clinical isolates of $A$. fumigatus have been observed to demonstrate increased expression of cyp $51 B .[38,39]$

The positive-regulation of cyp $51 A$ expression is controlled by the sterol regulatory element binding protein SrbA, which binds to the promoter region of cyp51A at two specific binding sites as a homodimer (Figure 1-4A). One of these two binding sites is negatively-regulated by both the multimeric CCAAT-binding complex (CBC) transcription factor (comprised of $\mathrm{HapB}, \mathrm{HapC}$, and $\mathrm{HapE}$ ) and the iron responsive basic region leucine zipper transcription factor, HapX. It is believed that under environmental conditions that favor repression of ergosterol biosynthesis and thus repression of steroldemethylase expression, the CBC and HapX cooperatively bind directly upstream of the 3' SrbA binding site and diminish the ability of SrbA to promote cyp51A expression. In the absence of either SrbA or the SrbA binding sites in the promoter region of cyp51A, cyp $51 \mathrm{~A}$ expression is essentially absent and $A$. fumigatus becomes hyper-susceptible to the triazoles.[26]

Two distinct mechanisms leading to overexpression of cyp51A have been identified in triazole-resistant clinical isolates of $A$. fumigatus. By far the most prevalent is the formation of a tandem repeat in the promoter region of cyp51A.[8, 27] Three 


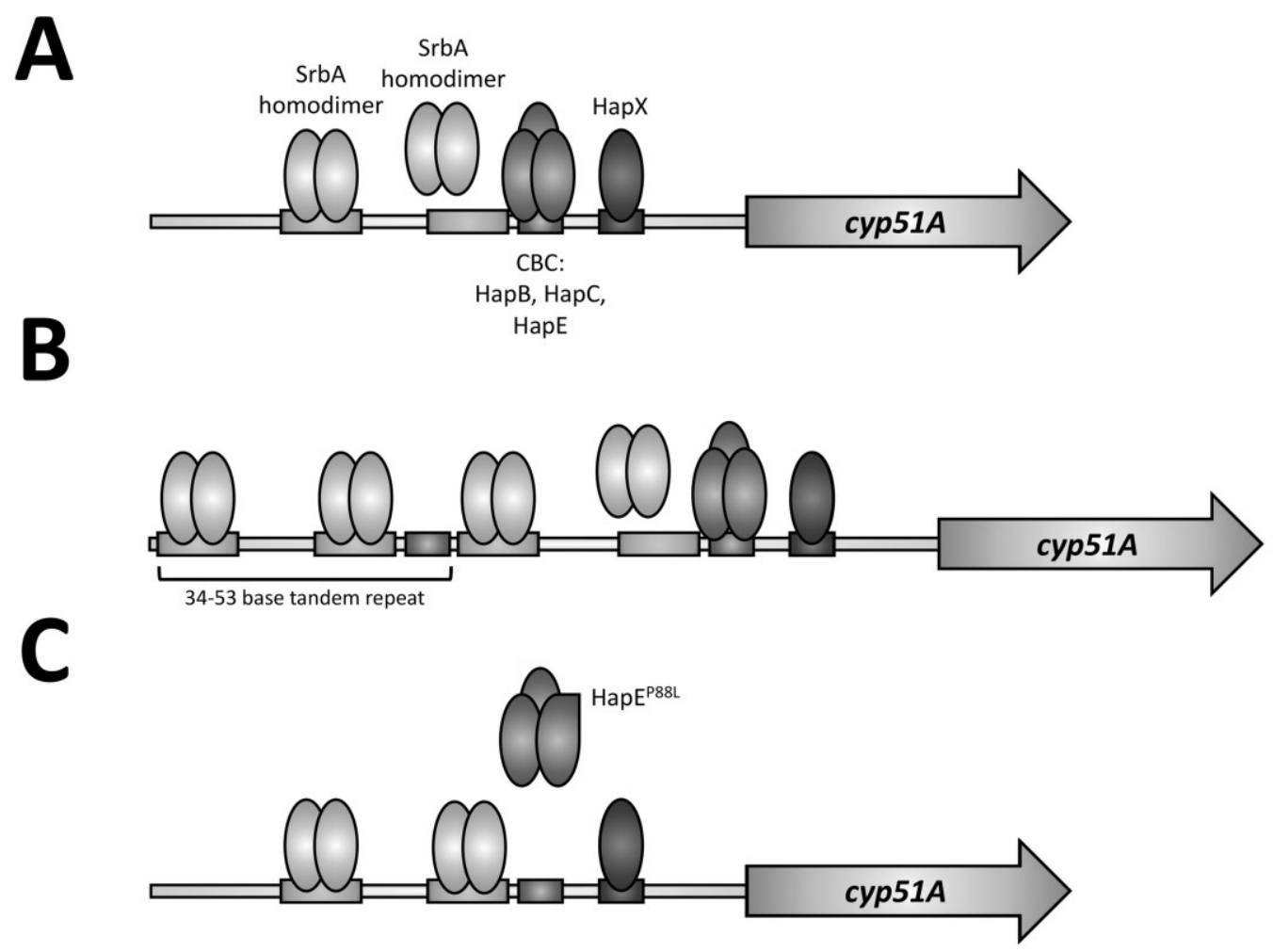

Figure 1-4. Regulation of cyp51A expression.

A) Regulation of cyp $51 A$ expression with SrbA homodimers under normal repression of CBC and HapX. B) Regulation of cyp51A expression in the presence of a tandem repeat mutation creating two additional SrbA binding sites not effectively repressed by the CBC and HapX. C) Regulation of cyp51A expression in the presence of CBC containing the mutant $\mathrm{HapE}^{\mathrm{P} 88 \mathrm{~L}}$, which is unable to effectively repress the SrbA mediated expression of cyp51A. 
versions of this tandem repeat sequence have been observed $\left(\mathrm{TR}_{34}, \mathrm{TR}_{46}, \mathrm{TR}_{53}\right)$. [33, 37 , 40] Each version differs in the length of the repeated sequence (34- 53 bases), but all result in the formation of two additional SrbA binding sites which are not effectively regulated by the CBC or HapX (Figure 1-4B). Thus, cyp51A expression is increased by 2-fold or more, and triazole MIC subsequently increase by approximately 4-fold.[26] Additionally, the $\mathrm{TR}_{34}$ and $\mathrm{TR}_{46}$ variations are, with only one known isolate being the exception, universally found in conjunction with specific mutations in the cyp51A gene itself, resulting in the amino acid substitutions L98H and Y121F/T289A, respectively.[32] Isolates possessing these mutations are often referred to as either having the $\mathrm{TR}_{34} / \mathrm{L} 98 \mathrm{H}$ or $\mathrm{TR}_{46} / \mathrm{Y} 121 \mathrm{~F} / \mathrm{T} 289 \mathrm{~A}$ genotype, and are most commonly observed to be multi- or pan-triazole-resistant. Worryingly, the prevalence of these tandem repeat mutations in both environmental and clinical isolates appears to be increasing around the world.[6, 8, 27, 37, 40]

In addition to alterations of the cyp $51 \mathrm{~A}$ promoter region, a single mutation in the hapE gene, encoding a subunit of the $\mathrm{CBC}$ multimer, has been shown to directly result in increased cyp5 $1 \mathrm{~A}$ expression. This mutation was originally identified in an isogenic pair of clinical isolates where resistance developed on triazole therapy, and results in a $\mathrm{P} 88 \mathrm{~L}$ amino acid substitution in HapE.[9] CBC containing the mutant $\mathrm{HapE}^{\mathrm{P} 88 \mathrm{~L}}$ subunit bind less effectively to the promoter region of cyp51A. Thus, cyp51A expression, driven by the unimpeded SrbA, is increased (Figure 1-4C). Similar to what is observed with the tandem repeat cyp51A genotypes, this mutation in hapE leads to a 2-fold or greater increase in cyp $51 \mathrm{~A}$ expression and increases in MICs of all triazole that have been tested.[26] While screening for this hapE mutation is not commonly performed, to date this mechanism of triazole resistance has only been identified in the original clinical isolates of $A$. fumigatus from which it was discovered.

\section{Overexpression of Drug Efflux Pumps}

In other pathogenic fungi, efflux pump overexpression has been shown to contribute to clinical triazole resistance. The A. fumigatus genome is predicted to contain more than 300 efflux pumps, many of which have been observed to have their expression induced by exposure to the triazoles.[25] Furthermore, a number of these pumps have been observed to be constitutively overexpressed among triazole-resistant clinical isolates.[25, 41] However, at present relatively little is known about which of these efflux pumps contribute to clinical triazole resistance. In triazole-susceptible laboratory strains

of $A$. fumigatus, the individual disruption of six drug efflux pump genes ( $a b c A, a b c C$, atr $F$, atrI, $m d r A$, and $m d r l$ ) has been observed to increase triazole susceptibility.[25, 4246] However, only the efflux pump gene, $a b c A$, has been demonstrated to contribute to triazole resistance when constitutively overexpressed in a triazole-susceptible laboratory strain of A. fumigatus.[45] Additionally, $a b c C$ is the only efflux pump gene which has been demonstrated to contribute to clinical triazole resistance. This ABC efflux pump of the G- subfamily is a close homolog of well characterized drug efflux pumps from other species of pathogenic fungi and has been observed to be overexpressed in a number of triazole-resistant clinical isolates of $A$. fumigatus. Disruption of $a b c C$ in a triazole- 
resistant clinical isolate of $A$. fumigatus decreased the itraconazole MIC by greater than 4- fold, while voriconazole and posaconazole susceptibility remained unchanged. This clinical isolate was observed to exhibit increased constitutive expression of $a b c C$ ( $>5$ fold) relative to the susceptible reference strain.[25] Drug efflux pumps, have therefore been proven to be a mechanism by which triazole resistance is mediated in clinical isolates of $A$. fumigatus. However, the genetic determinants of drug efflux pump overexpression in A. fumigatus remains elusive. Recently, the zinc-cluster transcription factor AtrR was found to promote the expression of both cyp51A and $a b c C$. AtrR is a close homolog of the Saccharomyces cerevisiae pleotropic drug response zinc-cluster transcription factor $P D R 1$, and $A$. fumigatus strains with atrR disrupted exhibit a dramatic reduction in expression of both $a b c C$ and cyp51A.[46] However, the presence of activating mutations in atrR among triazole-resistant clinical isolates has yet to be identified, and its direct role in clinical triazole resistance remains unclear.

\section{Emerging Mechanisms of Triazole Resistance}

In addition to the aforementioned canonical mechanisms of triazole resistance, additional potential pathways to resistance in A. fumigatus are beginning to be identified. The most notable of which are directly linked to the ergosterol biosynthetic pathway. Two different studies investigating mutations acquired in laboratory strains of triazolesusceptible A. fumigatus which develop triazole resistance upon triazole exposure have been successful in identifying novel resistance mechanisms. In these experiments, mutations in the hmgl gene were found to confer resistance to voriconazole, itraconazole, and posaconazole (isavuconazole was not tested). The $h m g l$ gene, encodes an $A$.

fumigatus homolog of 3-hydroxy-3-methyl-glutaryl (HMG) coenzyme A reductase, a key enzyme involved in both ergosterol and siderophore biosynthesis.[47] Additionally, a mutation in the cox10 gene, required for the normal biosynthesis of heme, was found to directly contribute to triazole resistance. Heme is utilized by a number of ergosterol biosynthetic genes, including cyp $51 \mathrm{~A}$ and $\operatorname{cyp} 51 \mathrm{~B}$, to catalyze enzymatic conversion of sterols. The mutation in cox 10 was observed to significantly reduce itraconazole susceptibility, yet voriconazole susceptibility was not clearly impacted.[48] While the roles of these mutations in clinical triazole resistance is currently unknown, these mutations serve to demonstrate the potential impact of non-canonical mechanisms on the clinical utility of the triazole antifungals and future screening for such mutations among clinical isolates of $A$. fumigatus may be merited.

\section{Epidemiology of Triazole Resistance}

Triazole resistance among A. fumigatus isolates emerged as early as the 1980's in immunocompromised patients from the United States receiving itraconazole as an investigational agent. Subsequently, resistant isolates were recovered from patients in the 1990's in the Netherlands and France. However, in each of these instances, the identification and reporting of triazole resistance followed years after the original clinical cases where the isolates were cultured. 
Today, analysis of the prevalence of triazole resistance among A. fumigatus remains limited by challenges in isolating Aspergillus from clinical specimens, as well as the additional resources and expertise required to perform antifungal susceptibility testing of molds.[49] However, triazole-resistant A. fumigatus are nonetheless being reported around the world in increasing numbers. [8, 50-53] A recent study examining the genetic diversity of $A$. fumigatus isolates observed that $6 \%$ of the 2,026 isolates obtained from 13 countries spanning four continents, were triazole-resistant.[54] This resistance appears to vary dramatically by both geographic region and patient population.

Both the increase in overall prevalence of triazole resistance and the variation in its distribution are perhaps best exemplified in epidemiology data from the Netherlands, where surveillance has been conducted since the first recovery of resistant clinical isolates. In the Netherlands, triazole resistance was originally observed to be present in $1.8 \%$ of A. fumigatus isolates collected from 1945 to 1998 . Subsequently, examination of resistance rates across seven Dutch university medical centers from 2007- 2009 found the average triazole resistance rate to have risen to $4.6 \%$, with medical center specific rates ranging from $0.8 \%$ to $9.5 \% .[7,51]$ No seasonal variation in resistance was observed and there was no clear geographic influence on the distribution of resistance. Importantly, the vast majority of triazole-resistant isolates were resistant to both itraconazole and voriconazole, and the case-fatality rate among patients with triazole-resistant disease was $88 \%$. Triazole resistance has also been observed to be more prevalent among certain high-risk patient populations. When resistance rates were tracked specifically among hematology patients at two Dutch medical centers, the prevalence of triazole resistance was found to be dramatically greater than that of the general population, ranging from 17 to $30 \%$.[55] Similar findings were also reported in a single center study of Dutch intensive care unit patients with invasive aspergillosis, where $26 \%$ of isolates were triazole-resistant.5 Overall 90-day mortality of patients with triazole-resistant disease was $100 \%$ in this population.

In the United States, triazole resistance among A. fumigatus is now being reported in isolates of both clinical and environmental origin. $[6,8,56]$ However, only one large, passive, surveillance study of triazole resistance among US A. fumigatus isolates has been performed to date. In this study 1,026 isolates were screened and $51(5 \%)$ were found to have itraconazole MIC above the EUCAST breakpoint for susceptible (CLSI has not published triazole breakpoints for A. fumigatus). [57] Voriconazole susceptibility was not reported. More recently, a study characterizing triazole-resistant isolates from the US performed extensive antifungal susceptibly testing on isolates collected from 2001 to 2015. Of 26 triazole-resistant $A$. fumigatus clinical isolates tested, all were resistant to both itraconazole and posaconazole (applying EUCAST breakpoints), and only seven and eight, respectively, were susceptible to isavuconazole and voriconazole. Additionally, although the use of agricultural sterol-demethylase inhibitors is less common in the US than other parts of the world, a study conducted by collecting environmental samples from peanut fields being treated with triazole agents found 56 of 200 recovered isolates $(28 \%)$ were triazole-resistant.[6] 
The distribution of identifiable mechanisms contributing to triazole resistance also appears to vary dramatically by geographic region. In the Netherlands, as well as other European and Asian countries such as the United Kingdom and India, the vast majority of triazole-resistant isolates share at least one common resistance mechanism, comprised of specific combinations of mutations in the sterol-demethylase gene cyp51A in conjunction with the formation of tandem repeats in the cyp $51 A$ promoter region $\left(\mathrm{TR}_{34} / \mathrm{L} 98 \mathrm{H}\right.$ or $\mathrm{TR}_{46} / \mathrm{Y} 121 \mathrm{~F} / \mathrm{T} 289 \mathrm{~A}$ genotype).[27] While many of these isolates likely possess additional resistance mechanisms, as suggested by the varying degrees of agent specific triazole resistance, the predominance of the $\mathrm{TR}_{34} / \mathrm{L} 98 \mathrm{H}$ and $\mathrm{TR}_{46} / \mathrm{Y} 121 \mathrm{~F} / \mathrm{T} 289 \mathrm{~A}$ genotypes among these resistant isolates cannot be overlooked. Conversely, in the United States only a small number of clinical isolates have been reported to possess this particular resistance mechanism. Of the 26 isolates in the aforementioned characterization of triazole-resistant $A$. fumigatus isolates, only two $\mathrm{TR}_{34} / \mathrm{L} 98 \mathrm{H}$ and two $\mathrm{TR}_{46} / \mathrm{Y} 121 \mathrm{~F} / \mathrm{T} 289 \mathrm{~A}$ genotype isolates were found. Yet, 16 isolates possessed other identifiable mutations in cyp51A. This discordance in prevalence of the $\mathrm{TR}_{34} / \mathrm{L} 98 \mathrm{H}$ and $\mathrm{TR}_{46} / \mathrm{Y} 121 \mathrm{~F} / \mathrm{T} 289 \mathrm{~A}$ genotypes has been linked to differences in the application of triazole related agricultural fungicides. Supporting this link, the $\mathrm{TR}_{34} / \mathrm{L} 98 \mathrm{H}$ and $\mathrm{TR}_{46} / \mathrm{Y} 121 \mathrm{~F} / \mathrm{T} 289 \mathrm{~A}$ genotypes are most prevalent among clinical and environmental samples from regions with higher utilization of these fungicides, and in vitro studies have demonstrated rapid acquisition of these and other cyp5 $1 \mathrm{~A}$ mutations upon exposure to agricultural triazoles. Notably, of the 56 triazole-resistant environmental samples from US peanut farms treated with triazoles, 21 (37.5\%) were found to possess the TR $\mathrm{TR}_{34} / \mathrm{L} 98 \mathrm{H}$ genotype.[6] This has led some experts to call for a reduction in the reliance on agricultural sterol-demethylase inhibitors in an effort to preserve the utility of the clinical agents.[58]

While the identification and study of potential risk factors for triazole-resistant aspergillosis has been limited by the general paucity of susceptibility data available for clinical isolates, a small number of patient populations have been associated with higher prevalence of triazole-resistant aspergillosis. Multiple studies have observed rates of triazole-resistant $A$. fumigatus isolates from hematology-oncology patients in Europe, approximating and even exceeding $20 \%$, however it is important to note that rates more similar to the general prevalence of resistance have been observed at other institutions. While prolonged use of prophylactic triazole is not uncommon in this patient population, these studies have also noted recovery of triazole-resistant isolates from patients with no prior triazole exposure, suggesting other patient factors possibly in conjunction with environmental acquisition of triazole-resistant isolates may be involved.[52, 55, 59, 60] Additionally, two epidemiologic studies examining the rates of triazole-resistant $A$. fumigatus among patients being treated in the intensive care units have also reported higher rates of resistance.[5, 59] Though both of these studies examined patients from medical centers in the Netherlands where environmental rates of triazole resistance are known to be high. It has been suggested that increased rates of triazole resistance among environmental isolates of $A$. fumigatus, may also increase the risk for triazole resistance among cases of aspergillosis.[61] This concept of environmental acquisition of resistant isolates is supported by a report of a patient admitted to the intensive care unit, with no prior history of antifungal drug exposure, suffering from proven invasive aspergillosis 
caused by an $A$. fumigatus isolate harboring the $\mathrm{TR}_{46} / \mathrm{Y} 121 \mathrm{~F} / \mathrm{T} 289 \mathrm{~A}$ genotype.

Environmental screening of the patient's home garden and household revealed multiple samples of a genetically indistinguishable and triazole-resistant $A$. fumigatus.[62]

\section{Conclusions}

The mold-active triazoles are essential to both prophylaxis and treatment of invasive aspergillosis, and few therapeutic alternatives exist. While the overall prevalence of triazole resistance among clinical isolates of $A$. fumigatus is currently observed to range between 1 and $10 \%$, these estimates are limited by a relative paucity of clinical samples with accompanying susceptibility data. Additionally, the recent dramatic increase in reports of resistant isolates from both clinical and environmental sources worldwide is of clear concern. Mutations in cyp51 A, overexpression of cyp5 $1 \mathrm{~A}$, and efflux pump gene overexpression all are known to contribute to resistance, yet much of the triazole resistance among $A$. fumigatus still remains unexplained. Also lacking is clinical experience with therapeutic options for the treatment of triazole-resistant $A$. fumigatus infections, and mortality associated with these infections remains unacceptably high. Thus, further research is greatly needed to both better understand the emerging threat of triazole-resistant $A$. fumigatus, and to develop novel therapeutic strategies to combat these resistant infections. 


\section{CHAPTER 2. CHALLENGING THE PARADIGM OF CLINICAL TRIAZOLE RESISTANCE IN ASPERGILLUS FUMIGATUS*}

\section{Introduction}

Aspergillus fumigatus is the predominant pathogen isolated from patients with invasive aspergillosis, the most common human invasive mold infection. Invasive aspergillosis is responsible for more than 200,000 life-threatening infections each year, and afflicts up to $10 \%$ of patients with acute leukemia as well as patients receiving stem cell or solid organ transplantation.[1, 60, 63] Unfortunately, the mortality rates associated with invasive aspergillosis remain unacceptably high, often exceeding 50\%, even when appropriately diagnosed and treated.[3-5, 60]

Contributing to the poor outcomes associated with the treatment of invasive aspergillosis is the relative paucity of clinically available antifungal agents with demonstrated clinical efficacy. Of the four major classes of antifungals, only the triazoles and amphotericin B are recommended as monotherapy for the treatment of aspergillosis, with the latter relegated to second-line or salvage treatment due to significant toxicities and the lack of oral formulations.[10] The triazole class of antifungals therefore have proven essential to the treatment of invasive aspergillosis, comprising both frontline treatment options such as voriconazole and isavuconazole, as well as salvage options such as posaconazole and itraconazole.[10] These agents are generally accepted to exert fungicidal activity against Aspergillus through the competitive inhibition of steroldemethylase, encoded by the genes cyp51A (Afu4g06890) and cyp51B (Afu7g03740) in $A$. fumigatus, which results in abrogation of the synthesis of ergosterol, the major fungal membrane sterol.[14, 15, 64, 65] However, the treatment of invasive aspergillosis has recently been further complicated by the global emergence of triazole-resistant disease. Over the past decade, triazole resistance among clinical isolates of $A$. fumigatus has been increasingly reported on six continents, with resistance rates exceeding $20 \%$ now being reported in some studies. $[6-8,30]$

Considerable research has led to the identification of three distinct molecular pathways to clinical triazole resistance in A. fumigatus. These three paths largely mirror the established mechanisms of resistance previously identified in other pathogenic fungi such as Candida albicans; 1) mutations in the sterol-demethylase gene cyp51A,2) overexpression of the sterol-demethylase gene cyp 51A, and 3) overexpression of the efflux pump-encoding gene $a b c C$ [9, 25, 26, 28, 31, 32, 34-36, 66]. However, it remains unknown to what extent these mechanisms explain the degree of triazole-resistance observed among clinical isolates of A. fumigatus, and resistant clinical isolates exhibiting

* Modified from final submission with permission. Rybak JM, Ge W, Wiederhold NP, Parker JE, Kelly SL, Rogers PD and Fortwendel JR. Mutations in hmgl, challenging the paradigm of clinical triazole resistance in Aspergillus fumigatus. MBio. 2019 Apr 2;10(2). pii: e00437-19. doi: 10.1128/mBio.00437-19. 
none of these three mechanisms have been repeatedly identified.[25] Thus, the molecular mechanisms underpinning much of the clinical resistance to this essential class of antiAspergillus agents remains unexplained, limiting the discovery of therapeutic strategies to overcome triazole-resistant aspergillosis.

In this work, we perform a comprehensive characterization of the direct contributions of previously identified mechanisms of triazole resistance in a large collection of triazole-resistant clinical isolates of $A$. fumigatus. We correct cyp $51 \mathrm{~A}$ mutations in 10 different triazole-resistant clinical isolates using a novel Cas9-mediated transformation system to delineate the direct impact these mutations have on clinical triazole susceptibility, and we assess the potential impact of overexpression of cyp51 $\mathrm{A}$, cyp $51 B$, and $a b c C$ on triazole susceptibility in a collection of 21 triazole-resistant clinical isolates. We subsequently demonstrate that these mechanisms alone fail to explain the resistance observed in this collection, suggesting the presence of additional mechanisms of clinical triazole resistance yet to be identified.

\section{Materials and Methods}

\section{Isolates, Media, and Growth Conditions Used}

Twenty-one previously characterized multi-triazole-resistant clinical isolates of $A$. fumigatus, and five triazole-susceptible control clinical isolates of A. fumigatus, originating from the United States were obtained from the Fungus Testing Laboratory at the University of Texas in San Antonio. The laboratory strains $a k u B^{\mathrm{KU} 80}$ and Af293 were obtained from the Fungal Genetics Stock Center. All strains and clinical isolates used in this study were maintained on glucose minimal media (GMM) agar at $37^{\circ} \mathrm{C}$. Transformants were selected for using sorbitol (1.2M) supplemented GMM agar (SMM) containing $150 \mathrm{mg} / \mathrm{L}$ of hygromycin. All conidia were harvested in sterile water from three-day-old growth plates and conidia were enumerated visually using a hemocytometer.

\section{Whole Genome Sequencing}

For each isolate, genomic DNA was extracted using the Qiagen DNeasy Plant Mini Kit as previously described.[67] DNA concentrations were quantified using both the Qubit Fluorometer and Nanodrop spectrophotometer using the manufacturers' protocols. Whole genome libraries were prepared and sequenced at the University of Tennessee Health Science Center Molecular Resource Center. Bioinformatics services were provided by code4DNA (www.code4DNA.com). Sequence reads for each sample were aligned using the STAR sequence alignment tool (v2.5.0b) to the A. fumigatus reference sequence (version A_fumigatus_Af293_version_s03-m05-r05) which was downloaded from http://aspgd.org/. Duplicate alignments were marked using Picard (v1.119) and variants were called using Freebayes (v1.1.0) using the haploid population-based model. 
The Freebayes output vcf file was split into individual sample vcf files using bcftools (v1.3) and variants for each sample were annotated with snpEff (v4.2) using the $A$. fumigatus reference sequence annotations.

\section{Construction of Promoter Replacement Repair Templates and Cas9-RNP for cyp51A, cyp51B, and abcC}

The plasmid pJMR2 was constructed by cloning the proximal $1 \mathrm{~kb}$ of the promoter sequence from the $A$. fumigatus heat shock protein-encoding gene, $h s p A$, into the plasmid pCR-HygR.[67] Then promoter replacement transformation repair templates for cyp51A, $c y p 51 B$, and $a b c C$ were created by PCR using primers (Table 2-1) which amplified both the hygromycin resistance cassette and the $h s p A$ promoter from the plasmid pJMR2, while also introducing approximately 40 bases of homology targeting sequences immediately upstream and downstream of the start codon for the respective gene of interest. PCR products were subsequently purified using the Gene Clean II Kit (MP Biomedicals). Cas9-RNP complexes targeting sequences immediately upstream of the open reading frame of each gene of interest were assembled as previously described.[67]

\section{Assessment cyp51A, cyp51B, and abcC Expression by RTqPCR}

For assessment of expression in clinical isolates, conidia from each isolate were allowed to germinate overnight in Aspergillus minimal media incubated at $37^{\circ} \mathrm{C}$ on an orbital shaker at 250 RPM. For assessment of expression in constitutive overexpression strains constructed in the $a k u B^{\mathrm{KU} 80}$ background, conidia from each isolate were allowed to germinate overnight in Aspergillus minimal media incubated at $37^{\circ} \mathrm{C}$ on an orbital shaker at 250 RPM, then transferred to fresh Aspergillus minimal media either with voriconazole $0.125 \mathrm{mg} / \mathrm{L}$ (treated) or without voriconazole (untreated) for an additional 6 hours at $37^{\circ} \mathrm{C}$ on an orbital shaker at $250 \mathrm{RPM}$. Then RNA was extracted from mature hyphae following liquid nitrogen crush as previously described.[68] The RevertAid RT kit (Thermo Scientific) was utilized to synthesize cDNA. PCR master mix and SYBR were utilized to amplify $A$. fumigatus cyp $51 A, c y p 51 B$, and $a b c C$ from cDNA by PCR per the manufacturer's instructions. Table 2-1 lists the gene specific primers used for PCR. Conditions used for PCR were as follows: AmpliTaq Gold activation at $95^{\circ} \mathrm{C}$ for 10 minutes, 40 cycles of denaturation at $95^{\circ} \mathrm{C}$ for 15 seconds and annealing/ extension at $60^{\circ} \mathrm{C}$. The dissociation curve and threshold cycle (CT) was determined using the CFX96 Real Time PCR system (Bio-Rad). Changes in gene expression among isolates were then calculated using the 2- $\Delta \Delta \mathrm{CT}$ method. All experiments were performed in triplicate from biological triplicates. As previously described, $\Delta \mathrm{CT}$ values were used to calculate the standard error.[68] Statistical analysis was performed using unpaired, two-tailed, students t-test in Prism 8 for Mac OS by GraphPad Software Inc. with significance set at 0.05 and degrees of freedom equal to 10 . 
Table 2-1. Oligonucleotides used for cyp51A, cyp51B, and abcC experiments.

\begin{tabular}{|c|c|c|c|}
\hline Oligo Type & Gene & Primer Name & Sequence (5'- 3') \\
\hline \multicolumn{4}{|c|}{ PCR Primers } \\
\hline & \multirow[t]{3}{*}{ cyp51A } & \multirow[t]{3}{*}{ cyp51A KI F CRISPR } & GAATACTGGGCAGCGGGCTGGAGATACT \\
\hline & & & ATGGCTTTCATATGTTGCTCAGCGTCAGC \\
\hline & & & \\
\hline & \multirow[t]{2}{*}{ cyp51A } & \multirow{2}{*}{ cyp51A KI R CRISPR } & $\begin{array}{l}\text { CCAGTAAGGTCTGAATAAGGGTTCAATA } \\
\text { CAGTCATTTATTAGGCCCTCGATTCGAGC }\end{array}$ \\
\hline & & & TCCCAAATCTGTCCAG \\
\hline & \multirow[t]{2}{*}{ cyp51A } & \multirow[t]{2}{*}{ cyp51A OE $h s p A \mathrm{~F}$} & САTTTCCСТCATCACTGCAACTCTAATCC \\
\hline & & & $\begin{array}{l}\text { TCGGGCTCACCCAGCTTGCATGCCTGCA } \\
\text { GG }\end{array}$ \\
\hline & \multirow[t]{2}{*}{ cyp51A } & \multirow[t]{2}{*}{ cyp51A OE hspA $\mathrm{R}$} & ACGGCCATGTAGGCCGTAAGCCATAGCA \\
\hline & & & $\begin{array}{l}\text { TCGGCACCATGCTTGGTACCTGTGAAGA } \\
\text { AGTG }\end{array}$ \\
\hline & \multirow[t]{2}{*}{ cyp51B } & \multirow[t]{2}{*}{ cyp51B OE hspA F } & СТАССТTTATTCCCTGCGACAGTAACTCG \\
\hline & & & $\begin{array}{l}\text { GTTGATCGATTTGAGCTTGCATGCCTGCA } \\
\text { GG }\end{array}$ \\
\hline & \multirow[t]{2}{*}{ cyp51C } & \multirow[t]{2}{*}{ cyp51B OE hspA $\mathrm{R}$} & TTGCAGATGCCGTCGAGAATGAACGCGA \\
\hline & & & $\begin{array}{l}\text { TGAGACCCATGCTTGGTACCTGTGAAGA } \\
\text { AGTG }\end{array}$ \\
\hline & \multirow[t]{2}{*}{$a b c C$} & \multirow[t]{2}{*}{$a b c C$ OE $h s p A \mathrm{~F}$} & GTTCGACTGTTTATGTGTATACCTATTGT \\
\hline & & & $\begin{array}{l}\text { GACGCAACCACTCAGCTTGCATGCCTGC } \\
\text { AGG }\end{array}$ \\
\hline & \multirow[t]{2}{*}{$a b c C$} & \multirow[t]{2}{*}{$a b c C$ OE $h s p A \mathrm{R}$} & GGGTTGATGTTAGGATTTATCGTCCCTAG \\
\hline & & & AAGAGACATGCTTGGTACCTGTGAAGAA \\
\hline & $h p h R$ & $h p h R$ screen $\mathrm{R}$ & CAAATCGCCCGCAGAAGCG \\
\hline \multicolumn{4}{|c|}{ crRNA Guide Sequences } \\
\hline & cyp $51 \mathrm{~A}$ & cyp51A 5' crRNA & GGCTTTCATATGTTGCTCAG \\
\hline & cyp $51 A$ & cyp51A 3' crRNA & TTCAAAGGATTTGGTGTGAT \\
\hline & cyp $51 \mathrm{~A}$ & cyp51B 5' OE crRNA & CTCCCTGTGTCTCCTCGAAA \\
\hline & cyp $51 B$ & cyp 51B 5' OE crRNA & TATTCCCTGCGACAGTAACT \\
\hline & $a b c C$ & $a b c C 5$, OE crRNA & GACTGAGTGTCTCACTCAAT \\
\hline \multicolumn{4}{|c|}{ RTqPCR Primers } \\
\hline & cyp $51 \mathrm{~A}$ & cyp51A RT F e1 & CTTCTTTGCGTGCAGAGA \\
\hline & cyp51A & cyp51A RT R e2 & GGGGTCGTCAATGGACTA \\
\hline & cyp $51 B$ & сур 51B RT F e1 & CTTTTTCGACTGCCGCGC \\
\hline & cyp $51 B$ & cyp51B RT R e2 & AGGCGTAGTGAGTGGAGA \\
\hline & $a b c C$ & $a b c C$ RT F e1 & CCTGGAGAAGGTCTCAATGTCG \\
\hline & $a b c C$ & $a b c C$ RT R e2 & GGCCAGACGTAGGCTCATCC \\
\hline
\end{tabular}




\section{Construction of Allele Replacement Repair Templates and Cas9-RNP for the cyp51A Locus}

The plasmid pCyp51A-HygR was created by cloning the proximal $1.5 \mathrm{~kb}$ of promoter sequence and open reading frame of the wild-type cyp51A allele from the genomic reference strain Af293, upstream of the hygromycin resistance cassette in the plasmid pCR-HygR, and then cloning the proximal $1.5 \mathrm{~kb}$ of the wild-type cyp $51 \mathrm{~A}$ terminator downstream of the hygromycin resistance cassette. Then, the cyp $51 A^{\mathrm{WT}}$ transformation repair template was created by PCR using primers which amplified the wild-type cyp5 $1 A$ allele (including 500 bases of promoter sequence), the hygromycin resistance cassette, and 500 bases of the cyp51A terminator. Cas9-RNP complexes targeting sequences immediately upstream and approximately 500 bases downstream of the open reading frame of cyp51A were assembled as previously described.[67] Target specific guide sequences are listed in Table 2-1.

\section{Aspergillus Protoplast Transformations}

Transformation of A. fumigatus protoplasts were performed as previously described with minor modifications.[67] Approximately $2 \mu \mathrm{g}$ of each portion applicable transformation repair templates were then mixed with $200 \mu \mathrm{L}$ of protoplasts, $26.5 \mu \mathrm{L}$ of Cas9-RNP complexes, and $25 \mu \mathrm{L}$ of polyethylene glycol (PEG)-CaCl2 buffer $(60 \%$ [wt/vol] PEG 3350, $50 \mathrm{mM} \mathrm{CaCl2} \cdot \mathrm{H} 2 \mathrm{O}, 450 \mathrm{mM}$ Tris-HCl, $\mathrm{pH}$ 7.5) as previously described. Following an incubation on ice for approximately one hour, $1.25 \mathrm{~mL}$ PEG$\mathrm{CaCl} 2$ was added and the mixture incubated for an additional 20 minutes at room temperature. The mixture was then diluted to a total volume of $3 \mathrm{~mL}$ with STC buffer (1.2 M sorbitol, $7.55 \mathrm{mM} \mathrm{CaCl} \cdot \mathrm{H} 2 \mathrm{O}, 10 \mathrm{mM}$ Tris-HCl, $\mathrm{pH} 7.5)$ and plated on SMM agar plates. Plates were then incubated overnight prior to being overlaid with SMM top agar (GMM supplemented with 1.2 M sorbitol and 0.7\% [wt/vol] agar) supplemented with hygromycin (final concentration of $150 \mathrm{~g} / \mathrm{ml}$ ), and the plates were incubated at $37^{\circ} \mathrm{C}$ for 3 days. Transformants were isolated to separate plates containing selective agar, genomic DNA extracted, and successful CRISPR/Cas9 editing confirmed by PCR screens using gene-specific primers and Sanger sequencing.

\section{Clinical Antifungal Susceptibility Testing}

Susceptibilities for amphotericin B, voriconazole, isavuconazole, itraconazole, and posaconazole were determined for all isolates in accordance with CLSI M38-A2 methodology utilizing broth microdilution in RPMI[69]. Each antifungal was obtained from the appropriate manufacturer. All agents were suspended in dimethyl sulfoxide (DMSO) for preparation of stock solutions. 


\section{Data Availability}

Whole genome sequencing data files for the 26 Aspergillus fumigatus isolates have been deposited to NCBI SRA under the accession number PRJNA491253.

\section{Results}

\section{Mutations in Sterol-Demethylase Alone Poorly Explain Clinical Triazole Resistance}

Mutations in the sterol-demethylase gene cyp $51 A$ are the most frequently identified mechanism of triazole resistance in A. fumigatus. [5, 8, 70] We sought to delineate the proportion of triazole resistance observed in individual clinical isolates explained by this mechanism. To accomplish this, a collection of 26 clinical isolates of $A$. fumigatus originating from the United States were obtained from the Fungus Testing Laboratory at the University of Texas Health Science Center at San Antonio, including 21 multi-triazole-resistant isolates with previously reported clinical origins and characterized cyp51A genotypes, and 5 triazole-susceptible control isolates.[8] High quality genomic DNA was extracted from each of the 26 isolates, whole genome sequencing was performed targeting a read depth of approximately 100 reads per base aligned to the Af293 A. fumigatus reference genome assembly, and a total of 48,552 SNPs, insertion or deletions (indels) unique to triazole-resistant isolates were identified within 8,669 genes with annotations available. Of the 21 triazole-resistant isolates in this collection, 16 were identified as possessing peptide sequence altering cyp $51 \mathrm{~A}$ mutations unique to resistant isolates. Conversely, no unique cyp $51 B$ mutations were identified. We selected 10 of these 16 isolates with cyp $51 A$ mutations, which also exhibited resistance to all clinically available triazole agents, for correction of the cyp $51 \mathrm{~A}$ sequence to that of the wild-type consensus sequence. These isolates included examples of previously characterized cyp $51 A$ mutations $\left(\mathrm{TR}_{34} / \mathrm{L} 98 \mathrm{H}, \mathrm{TR}_{46} / \mathrm{Y} 121 \mathrm{~F} / \mathrm{T} 289 \mathrm{~A}, \mathrm{G} 448 \mathrm{~S}\right)$, mutations at residues where alternative mutations have previously been characterized (G138C and F219S), and mutations never before characterized to our knowledge (M263I and I367F) (Table 2-2).[28, 34, 35, 48, 71]

In order to test the direct contribution of these mutations to clinical triazole resistance in these isolates, the wild-type cyp51A promoter region and open reading frame from the triazole-susceptible reference strain Af293 was first cloned upstream of the hygromycin resistance cassette in plasmid pCR-HygR.[67] 1500 bases of the cyp51A terminator was then cloned downstream of the hygromycin resistance cassette, yielding the plasmid pCyp51A-HygR. Then, the cyp5 $1 A^{\mathrm{WT}}$ transformation repair template was created by PCR using primers which amplified the wild-type cyp51A allele (including 500 bases of promoter sequence), the hygromycin resistance cassette, and 500 bases of the cyp $51 A$ terminator. Importantly, this repair template extends approximately 200 bases upstream of the promoter region where characterized regulatory elements and tandem repeat sequences have been identified.[26] Protoplasts of each of the 10 triazole-resistant clinical isolates, as well as the well-characterized triazole-susceptible laboratory strain 
Table 2-2. Triazole MIC of clinical isolates and derivative $c y p 51 A^{\mathrm{WT}}$ strains.

\begin{tabular}{|c|c|c|c|c|}
\hline \multirow{2}{*}{$\begin{array}{l}\text { Parent Clinical Isolate or } \\
\text { Derivative cyp } 51 A^{\mathrm{WT}} \text { Strain }\end{array}$} & \multirow[b]{2}{*}{ cyp $51 A$ Genotype } & \multicolumn{3}{|c|}{ Triazole MIC $(\mathrm{mg} / \mathrm{L})$} \\
\hline & & Voriconazole & Itraconazole & Posaconazole \\
\hline DI15-117 & $\mathrm{F} 219 \mathrm{~S}$ & 2 & $\geq 32$ & 2 \\
\hline DI15-117 cyp51A WT & WT & 4 & $\geq 32$ & 4 \\
\hline DI15-100 & M263I & 8 & $\geq 32$ & 1 \\
\hline DI15-100 cyp51A ${ }^{\mathrm{WT}}$ & WT & 8 & $\geq 32$ & 0.5 \\
\hline DI15-116 & $\mathrm{TR}_{34} / \mathrm{L} 98 \mathrm{H}$ & 8 & $\geq 32$ & 1 \\
\hline DI15-116 cyp51A WT & WT & 1 & 0.25 & 0.125 \\
\hline DI15-102 & $\mathrm{TR}_{34} / \mathrm{L} 98 \mathrm{H}$ & 8 & $\geq 32$ & 2 \\
\hline DI15-102 cyp51A ${ }^{\mathrm{WT}}$ & WT & 4 & $\geq 32$ & 0.5 \\
\hline DI15-108 & G138C & 4 & $\geq 32$ & 1 \\
\hline DI15-108 cyp51A ${ }^{\mathrm{WT}}$ & WT & 4 & $\geq 32$ & 0.125 \\
\hline DI15-95 & M263I & 16 & $\geq 32$ & 2 \\
\hline DI15-95 cyp51A $A^{\mathrm{WT}}$ & WT & 16 & $\geq 32$ & 1 \\
\hline DI15-98 & $\mathrm{I} 367 \mathrm{~F}$ & 16 & 16 & 2 \\
\hline DI15-98 cyp51A $A^{\mathrm{WT}}$ & WT & 16 & $\geq 32$ & 1 \\
\hline DI15-96 & $\mathrm{TR}_{46} / \mathrm{Y} 121 \mathrm{~F} / \mathrm{T} 289 \mathrm{~A}$ & $\geq 32$ & 4 & 1 \\
\hline DI15-96 cyp51A $A^{\mathrm{WT}}$ & WT & $\geq 32$ & 0.5 & 0.25 \\
\hline DI15-106 & $\mathrm{TR}_{46} / \mathrm{Y} 121 \mathrm{~F} / \mathrm{T} 289 \mathrm{~A}$ & $\geq 32$ & 4 & 1 \\
\hline DI15-106 cyp51A $\mathrm{WT}$ & WT & 8 & 2 & 0.5 \\
\hline DI15-120 & G448S & $\geq 32$ & $\geq 32$ & 1 \\
\hline DI15-120 cyp51A ${ }^{\mathrm{WT}}$ & WT & $\geq 32$ & $\geq 32$ & 1 \\
\hline
\end{tabular}

MIC shown in bold are greater than epidemiologic cutoff values as published by the Clinical Laboratory and Standards Institute $(1 \mathrm{mg} / \mathrm{L}$ for voriconazole and itraconazole; $0.25 \mathrm{mg} / \mathrm{L}$ for posaconazole). WT: cyp $51 \mathrm{~A}$ sequence matches the Af293 wild-type consensus sequence. 
$a k u B^{\mathrm{KU} 80}$ (CEA17 $\Delta a k u B^{\mathrm{KU} 80}$ ) which has a wild-type cyp51A allele, were then transformed with Cas9-ribonucleoprotein (RNP) complexes, consisting of Cas9 protein and crRNA-trRNA duplexes targeting sequences immediately upstream and downstream of the cyp $51 \mathrm{~A}$ locus. The resulting transformants were selected on Aspergillus minimal media containing hygromycin as previously described.[67, 72] Integration of the repair template at the native cyp51A locus was confirmed by PCR, and acquisition of the cyp $51 A^{\mathrm{WT}}$ sequence was verified by Sanger sequencing.

Clinical susceptibility testing was performed for each of the 10 triazole-resistant clinical isolates and the corresponding $\operatorname{cyp} 51 A^{\mathrm{WT}}$ derivative strains for voriconazole, itraconazole, and posaconazole according to the Clinical Laboratory Standards Institute M38-A2 standards (Table 1).[69] All of the resistant clinical isolates exhibited triazole minimum inhibitory concentrations (MIC) consistent with those previously reported for these isolates under clinical susceptibility testing conditions, and all MIC for each agent tested were above epidemiologic cut-off values previously reported by CLSI $(1 \mathrm{mg} / \mathrm{L}$ for voriconazole and itraconazole, $0.25 \mathrm{mg} / \mathrm{L}$ for posaconazole). $[8,73]$ Among the cyp $51 A^{\text {WT }}$ strains, only one strain (DI15-116 cyp $51 A^{\mathrm{WT}}$ ) was observed to have full restoration of wild-type triazole susceptibility, and two strains (DI15-96 cyp51A ${ }^{\mathrm{WT}}$ and DI15-108 cyp $51 A^{\mathrm{WT}}$ ) were observed to have restoration of wild-type susceptibility to at least one triazole. Additionally, two strains (DI15-102 cyp51A $A^{\mathrm{WT}}$ and DI15-106 cyp $51 A^{\mathrm{WT}}$ ) were observed to have at least one triazole MIC decrease by more than one dilution. Five of the 10 cyp $51 A^{\mathrm{WT}}$ derivative strains exhibited no change in susceptibility to any triazole agent tested. Importantly, replacement of the native wildtype cyp51A allele with the cyp $51 A^{\mathrm{WT}}$ cassette in the $a k u B^{\mathrm{KU} 80}$ background had no effect on triazole MIC. Taken together, these data demonstrate that while mutations in cyp51A are common among triazole-resistant clinical isolates of $A$. fumigatus, and a number of these mutations have been demonstrated to increase triazole MIC when introduced into a triazole susceptible background, mutations in cyp $51 \mathrm{~A}$ alone poorly explain the high level of triazole resistance observed in this collection of clinical isolates.

\section{Analysis of the Expression of the Sterol-Demethylases Genes cyp51A and cyp51B Among Triazole-Resistant Clinical Isolates.}

Triazole-resistant clinical isolates of A. fumigatus exhibiting overexpression of cyp $51 A$ and cyp $51 B$, relative to susceptible comparator isolates, have previously been reported.[25, 39] Additionally, both the formation of tandem repeats $\left(\mathrm{TR}_{34}, \mathrm{TR}_{46}\right.$, and $\mathrm{TR}_{53}$ ) in the promoter region of cyp51A and a mutation (encoding the amino acid substitution P88L) in hapE, a CCAAT-binding complex protein-encoding gene which participates in the negative regulation cyp $51 \mathrm{~A}$ expression, have been shown to increase both cyp51A expression and triazole MICs.[9, 26, 34] Following the observation that mutations in cyp5 $1 \mathrm{~A}$ alone do not explain the degree of triazole resistance observed in this collection of clinical isolates, we sought to evaluate the potential contributions of sterol-demethylase gene overexpression to clinical triazole resistance. To accomplish this, each of the 26 clinical isolates in our collection were grown overnight at $37{ }^{\circ} \mathrm{C}$ in Aspergillus minimal media in biological triplicate, and RNA was extracted from mature 
hyphae following liquid nitrogen crush as previously described.[68] Reverse transcription quantitative-PCR (RTqPCR) was then utilized to quantify the relative expression of both cyp $51 A$ and cyp $51 B$ in each of the 21 triazole-resistant clinical isolates as compared to the expression level observed among the 5 triazole-susceptible control isolates.

The expression of cyp51A and cyp $51 B$ in all clinical isolates was compared to the triazole-susceptible isolate DI16-6 (voriconazole MIC $0.5 \mathrm{mg} / \mathrm{L}$ ). Among triazolesusceptible control isolates, the expression of cyp $51 \mathrm{~A}$ ranged from 0.5 to 1.6 -fold that of isolate DI16-6 (Figure 2-1A), with isolates DI16-5, DI-16-7, and DI16-9 exhibiting statistically significant lower expression of cyp $51 \mathrm{~A}$. While the expression of cyp $51 \mathrm{~A}$ among triazole-resistant clinical isolates ranged from 0.7 to 3.5 -fold that of the susceptible comparator isolate. Twelve of the 21 triazole-resistant isolates were observed to have cyp $51 A$ expression that exceeded the highest expression level observed among triazole-susceptible isolates. Of these 12 isolates, 8 were found to have cyp $51 \mathrm{~A}$ expression levels statistically greater than the susceptible comparator isolate. This included 2 isolates (DI15-102 and DI15-116) which possess the TR $34 / \mathrm{L} 98 \mathrm{H}$ cyp51A mutation, 2 isolates (DI15-96 and DI15-106) which possess the TR $46 / \mathrm{Y} 121 \mathrm{~F} / \mathrm{T} 289 \mathrm{~A}$ cyp 51A mutation, and one isolate (DI15-105) which possesses the previously characterized P88L-encoding mutation in hapE. However, no previously characterized mutations known to contribute to cyp $51 \mathrm{~A}$ overexpression, were identified in the remaining 3 isolates. The expression of cyp $51 B$ among the triazole-susceptible control isolates ranged from 0.7 to 1.3-fold that of isolate DI16-6 (Figure 2-1B), with isolate DI16-8 exhibiting statistically greater expression of cyp $51 B$ ( 1.3 fold). The expression of cyp $51 B$ among triazole-resistant clinical isolates ranged from 0.7 to 2.1 -fold that of the susceptible comparator isolate, with 4 isolates (DI15-95, DI15-97, DI15-102 and DI15108) exhibiting expression of cyp $51 B$ exceeding that of the highest expression level observed among triazole-susceptible isolates, 3 of which (DI15-95, DI15-102 and DI15108) were statistically significant.

\section{Constitutive Overexpression of cyp51B Decreases Triazole Susceptibility}

Overexpression of cyp $51 \mathrm{~A}$ has previously been shown to contribute to triazole resistance, but it remains unknown if overexpression of the paralogous gene, cyp $51 B$, has a similar effect on triazole susceptibility. We sought to employ a novel Cas9-RNP mediated promoter replacement system to delineate the direct impact of constitutive overexpression of cyp51B on triazole MIC. To accomplish this, the previously characterized, strong, constitutive promoter from the heat shock protein encoding gene, hspA (Afulg07440), was cloned upstream of the hygromycin resistance cassette in the plasmid pCR-HygR.[74] A transformation repair template for the cyp51B promoter replacement was then constructed by amplifying the $h s p A$ promoter and hygromycin resistance cassette from the resulting plasmid (pJMR2) with primers that introduced approximately 50 bases of homology for the 3 ' end of the native promoter region and the 5 ' end of the cyp51B open reading frame. Protoplasts of the well-characterized triazole- 
Figure 2-1. Relative expression of cyp51A and cyp51B among triazole-resistant clinical isolates.

A) Expression of cyp $51 \mathrm{~A}$ in clinical isolates relative to triazole-susceptible isolate DI166 , identified mutations known to contribute to cyp51A overexpression are shown on the corresponding bar $\mathbf{B}$ ) expression of $c y p 51 B$ in clinical isolates relative to triazolesusceptible isolate DI16-6; Triazole-susceptible isolates shown as open bars, triazoleresistant isolates shown as filled bars. Triazole-resistant isolates shown in order of escalating voriconazole MIC from left to right. Horizontal dotted lines denote highest and lowest expression level observed among the 5 triazole-susceptible isolates. Comparisons with the susceptible control isolate DI16-6 with statistically significant $(\mathrm{p}<0.05)$ results are noted with an Asterix. 

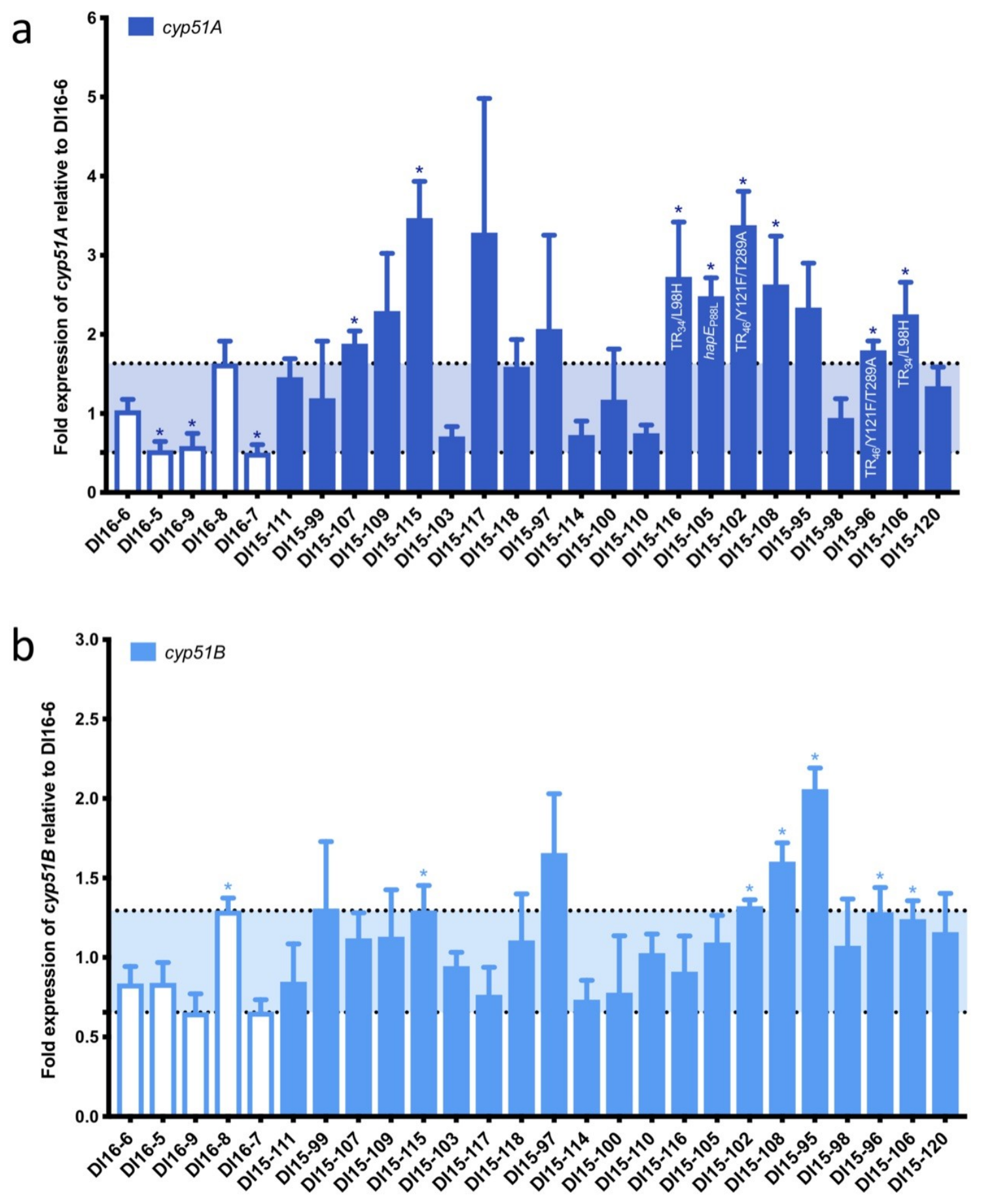
susceptible laboratory strain $a k u B^{\mathrm{KU} 80}$ were then transformed with Cas9-RNP complexes, consisting of Cas9 protein and crRNA-trRNA duplexes targeting sequences immediately upstream of the cyp $51 B$ start codon, yielding the strain $a k u B^{\mathrm{KU} 80} P_{h s p A c y p} 51 B$ (Figure 2-2).[75] As a comparator, the same methodology was employed to create the constitutive cyp $51 A$ overexpressing strain, $a k u B^{\mathrm{KU} 80} P_{h s p A c y p} 51 A$.

The relative expression of both cyp51A and cyp51B in the strains $a k u B^{\mathrm{KU} 80} P_{h s p A} c y p 51 A$ and $a k u B^{\mathrm{KU} 80} P_{h s p A c y p} 51 B$ was then compared to the parental $a k u B^{\mathrm{KU} 80}$ strain under both normal growth conditions and following voriconazole treatment. Conidia from each strain were growth in Aspergillus minimal media at $37^{\circ} \mathrm{C}$ until germination, and then were transferred to fresh Aspergillus minimal media containing either no voriconazole, or $0.125 \mathrm{mg} / \mathrm{L}$ of voriconazole (half of the MIC of the parental $a k u B^{\mathrm{KU} 80}$ strain) for an additional 6 hours at $37^{\circ} \mathrm{C}$. RNA was extracted from each sample as previously described and RTqPCR was performed. The expression of cyp $51 B$ in $a k u B^{\mathrm{KU} 80}$ was 2-fold higher after treatment with voriconazole than in untreated conditions (Figure 2-3A). Expression of cyp $51 B$ in the $a k u B^{\mathrm{KU} 80} P_{h s p A}$ cyp $51 B$ strain was 35.9-fold higher under untreated conditions, and 36.9-fold higher following voriconazole exposure, as compared to the expression measured in the parental $a k u B^{\mathrm{KU} 80}$ under untreated conditions. This increased expression was statistically greater than in the parental strain under both treated and untreated conditions. The expression of cyp $51 B$ in the $a k u B^{\mathrm{KU} 80} P_{h s p A c y p} 51 A$ strain approximated that of $a k u B^{\mathrm{KU} 80}$ under both untreated and voriconazole-treated conditions. The expression of cyp $51 A$ in $a k u B^{\mathrm{KU} 80}$ was 4.1 -fold higher following treatment with voriconazole as compared to untreated conditions. Expression of cyp $51 A$ in the $a k u B^{\mathrm{KU} 80} P_{h s p A c y p} 51 A$ strain was 14.1 -fold higher under untreated conditions, and 18.5-fold higher following voriconazole exposure, as compared to the expression measured in the parental $a k u B^{\mathrm{KU} 80}$ under untreated conditions. This increased expression was statistically greater than in the parental strain under both treated and untreated conditions. The expression of cyp $51 A$ in the $a k u B^{\mathrm{KU} 80} P_{h s p A} c y p 51 B$ strain was similar to that of $a k u B^{\mathrm{KU} 80}$ under both untreated and voriconazole-treated conditions.

Upon confirmation of the constitutive overexpression of cyp $51 A$ and cyp $51 B$ in the $a k u B^{\mathrm{KU} 80} P_{h s p A c y p} 51 A$ and $a k u B^{\mathrm{KU} 80} P_{h s p A c y p} 51 B$ strains, respectively, voriconazole MIC were performed in triplicate according to CLSI M38-A2 standards.[69] The parental $a k u B^{\mathrm{KU} 80}$ strain exhibited a voriconazole MIC of $0.25 \mathrm{mg} / \mathrm{L}$. The $a k u B^{\mathrm{KU} 80} P_{h s p A c y p} 51 A$ strain was observed to have a 2-fold increase in voriconazole MIC $(0.5 \mathrm{mg} / \mathrm{L})$, which is similar to the decrease in susceptibility previously reported when expression of this gene is increased as a result of either the formation of tandem repeats in the cyp $51 \mathrm{~A}$ promoter or a mutation in hapE (Figure 2-3B)[9, 26, 34, 35]. Interestingly, the voriconazole MIC of the $a k u B^{\mathrm{KU} 80} P_{h s p A}$ cyp $51 B$ strain was $1 \mathrm{mg} / \mathrm{L}, 4$-fold higher than the parental isolate. Taken together, these results demonstrate that overexpression of either steroldemethylase gene can decrease triazole susceptibility. However, even when the expression of either of these genes increase by as much as 36.9-fold, MIC did not exceed the epidemiological cutoff-value for voriconazole reported by CLSI (1mg/L).[73] 


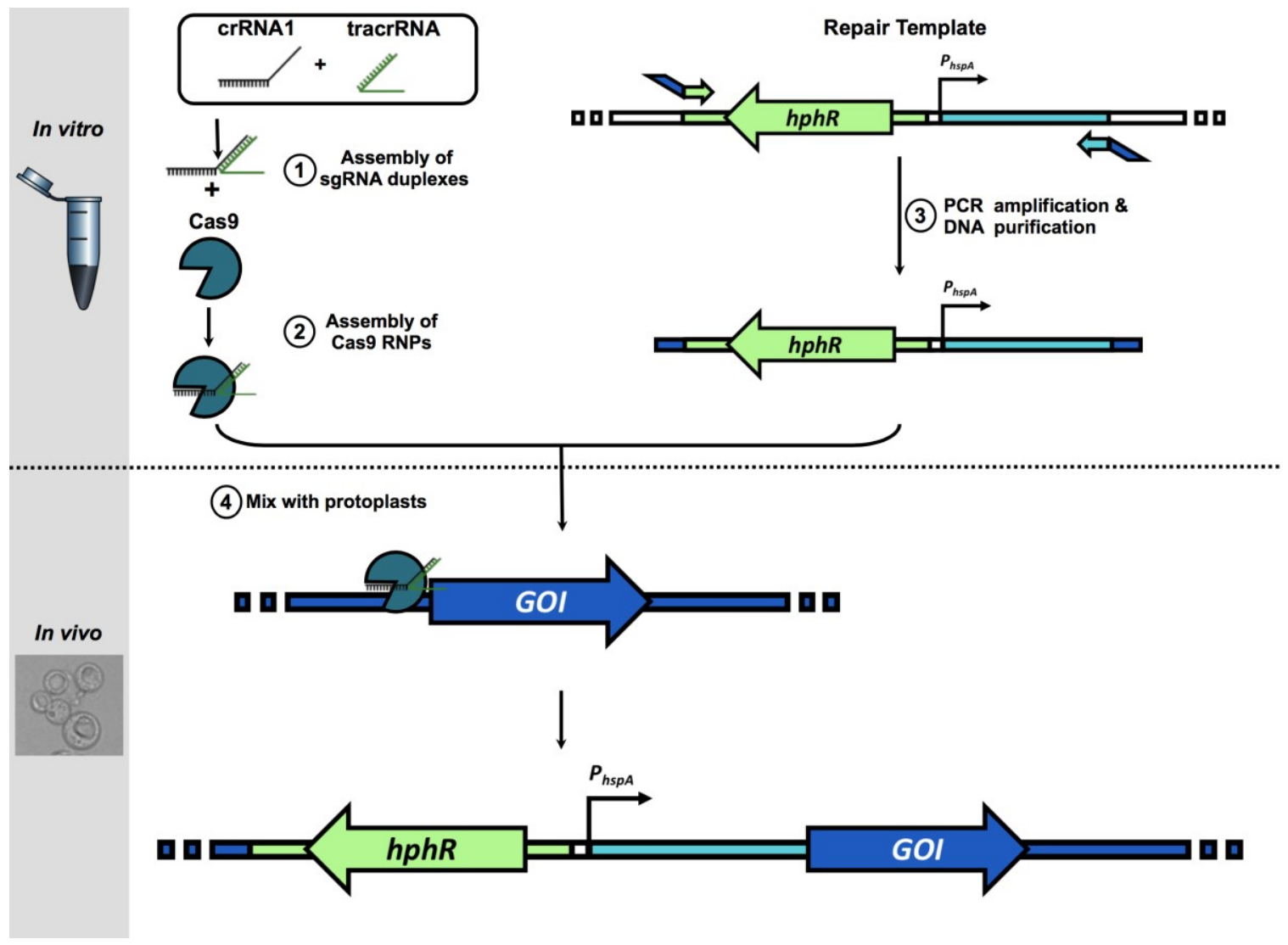

Figure 2-2. Schematic of Cas9-RNP mediated promoter replacement system. Constitutive overexpression repair templates were amplified from pJMR2 using primers incorporating microhomology targeting genes of interest (GOI), then mixed with Cas9RNP targeting immediately upstream of the start codon of the GOI, and protoplasts created from the strain $a k u B^{\mathrm{KU} 80}$. 


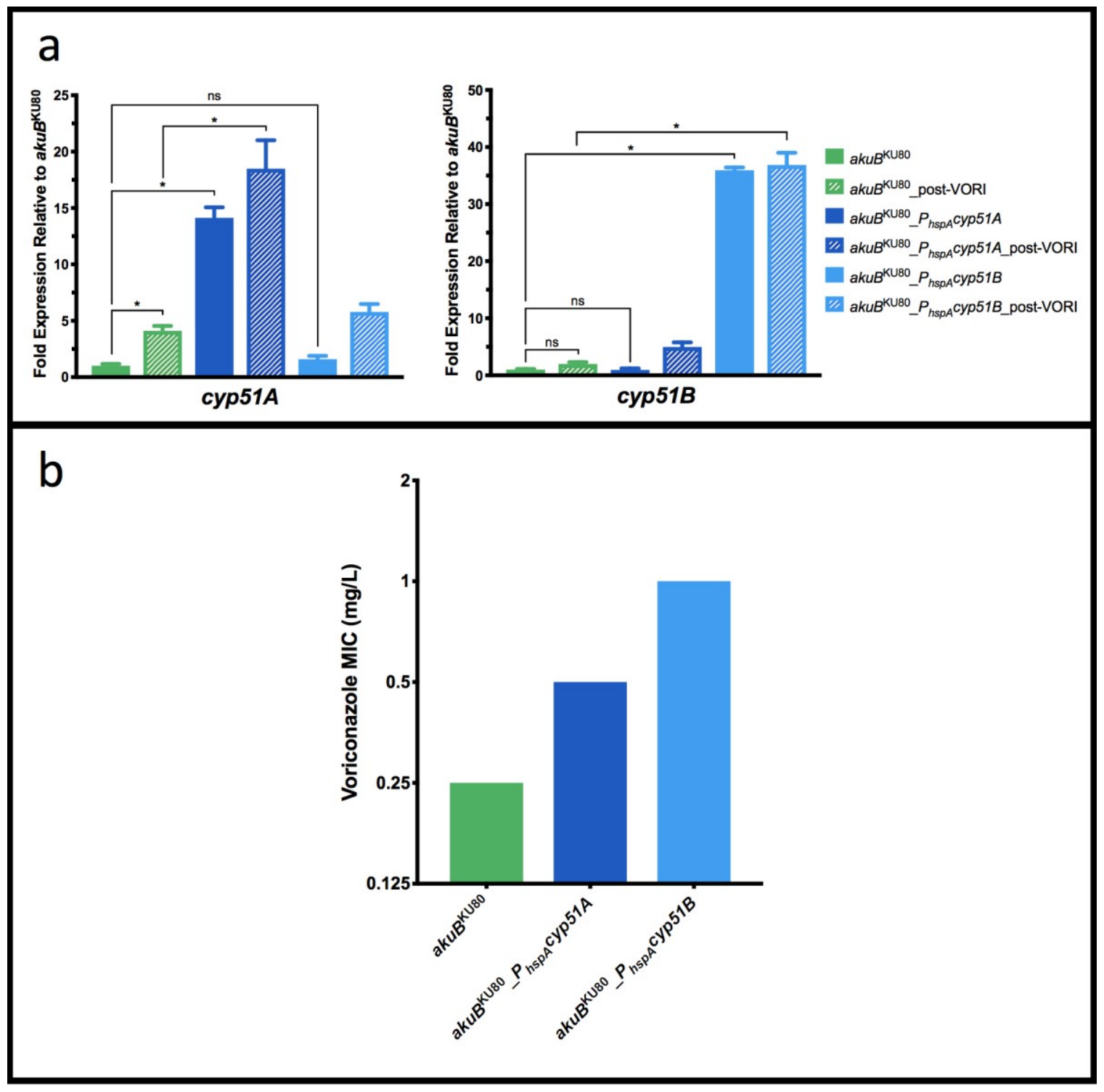

Figure 2-3. Relative expression of $c y p 51 A$ and $c y p 51 B$, and voriconazole MIC in promoter replacement strains $a k u B^{\mathrm{KU} 80} P_{h s p A c y p 51 A}$ and $a k u B^{\mathrm{KU80}} P_{h s p A c y p 51 B}$. The expression of A) cyp $51 A$ and cyp $51 B$ in $a k u B^{\mathrm{KU} 80}$, akuBKU80 $P_{h s p A c y p} 51 A$, and $a k u B^{\mathrm{KU} 80} P_{h s p A}$ cyp $51 B$ was determined by RTqPCR following growth in Aspergillus minimal media at $37^{\circ} \mathrm{C}$ both with voriconazole $(0.125 \mathrm{mg} / \mathrm{L})$ treatment for 6 hours, and without voriconazole treatment. The expression level for each sample is shown relative to that of the respective gene in $a k u B^{\mathrm{KU} 80}$ without voriconazole treatment. B) Voriconazole MIC for $a k u B^{\mathrm{KU} 80}, a k u B^{\mathrm{KU} 80} P_{h s p A} c y p 51 A$, and $a k u B^{\mathrm{KU} 80} P_{h s p A} c y p 51 B$. Comparisons with statistically significant $(\mathrm{p}<0 . \overline{0} 5)$ results are noted with an Asterix, while those that are not are noted with "ns". 


\section{Analysis of the Expression of the Efflux Pump-Encoding Gene abcC Among Triazole-Resistant Clinical Isolates}

In addition to sterol-demethylase-mediated mechanisms of triazole resistance, increased constitutive expression of the ATP-binding cassette efflux pump encoding gene, $a b c C$ (Afulg14330; also known as $a b c B, c d r 1 B$, and $a b c G 1$ ), has been associated with triazole resistance in $A$. fumigatus. [25, 48, 66] Furthermore, deletion of $a b c C$ in both triazole-susceptible laboratory strains of $A$. fumigatus, as well as a triazole-resistant clinical isolate, has been shown to increase triazole susceptibility.[25, 48, 66] As cyp51A and cyp $51 B$-mediated mechanisms alone do not adequately explain the high level of triazole-resistance in this collection of clinical isolates, we sought to characterized the expression of $a b c C$ and its potential impact on triazole susceptibility. To accomplish this, each of the 26 clinical isolates in our collection were grown overnight at $37^{\circ} \mathrm{C}$ in Aspergillus minimal media in biological triplicate, and RNA was extracted as previously described. RTqPCR was then utilized to quantify the relative expression of $a b c C$ in each of the 21 triazole-resistant clinical isolates as compared to the expression level observed among the 5 triazole-susceptible control isolates.

The expression of $a b c C$ in all clinical isolates was compared to the triazolesusceptible isolate DI16-6. Among triazole-susceptible control isolates, the expression of $a b c C$ ranged from 0.7 to 2-fold that of isolate DI16-6 (Figure 2-4A), with both isolates DI16-5 and DI16-8 exhibiting statically greater $a b c C$ expression (1.3 and 2.0-fold respectively) than DI16-6. The expression of $a b c C$ among triazole-resistant clinical isolates ranged from 1.2 to 33.2 -fold that of the susceptible comparator isolate. Nineteen of the 21 triazole-resistant isolates were observed to have $a b c C$ expression that was statistically greater than the expression observed in the susceptible control isolate DI16-6, 14 of which exhibited $a b c C$ expression which also exceeded the highest expression level observed among triazole-susceptible isolates (2-fold). While the majority of these 14 isolates exhibited modestly elevated $a b c C$ expression (2.4 to 4.1-fold that of the comparator susceptible isolate) isolates DI15-110, DI15-120, and DI15-106 exhibited markedly higher levels of $a b c C$ expression (6.5, 10.4, and 33.2-fold, respectively). Notably, the pan-triazole-resistant isolate DI15-110, which exhibited the highest level of $a b c C$ expression, possesses no mutations in cyp51 $A$ and did not exhibit increased constitutive expression of either sterol-demethylase gene.

As previous studies associating increased triazole resistance to $a b c C$ have entirely relied upon deletion of the $a b c C$ gene among either clinical or laboratory isolates of $A$. fumigatus, we next sought to utilize our Cas9-RNP mediated promoter replacement system to delineate the direct impact of constitutive overexpression of $a b c C$ on triazole MIC. To accomplish this, protoplasts of the triazole-susceptible laboratory strain $a k u B^{\mathrm{KU} 80}$ were transformed with Cas9-ribonucleoprotein (RNP) complexes, consisting of Cas9 protein and crRNA-trRNA duplexes targeting sequences immediately upstream of the $a b c C$ start codon, as well as the $P_{h s p A} a b c C$ transformation repair template which contains both the $h s p A$ promoter and hygromycin resistance cassette flanked by 50-bases of micro-homology targeting the $a b c C$ locus, yielding the strain $a k u B^{\mathrm{KU} 80} P_{h s p A} a b c C$.

Subsequently, the relative expression of $a b c C$ in both $a k u B^{\mathrm{KU} 80} P_{h s p A} a b c C$ and the 


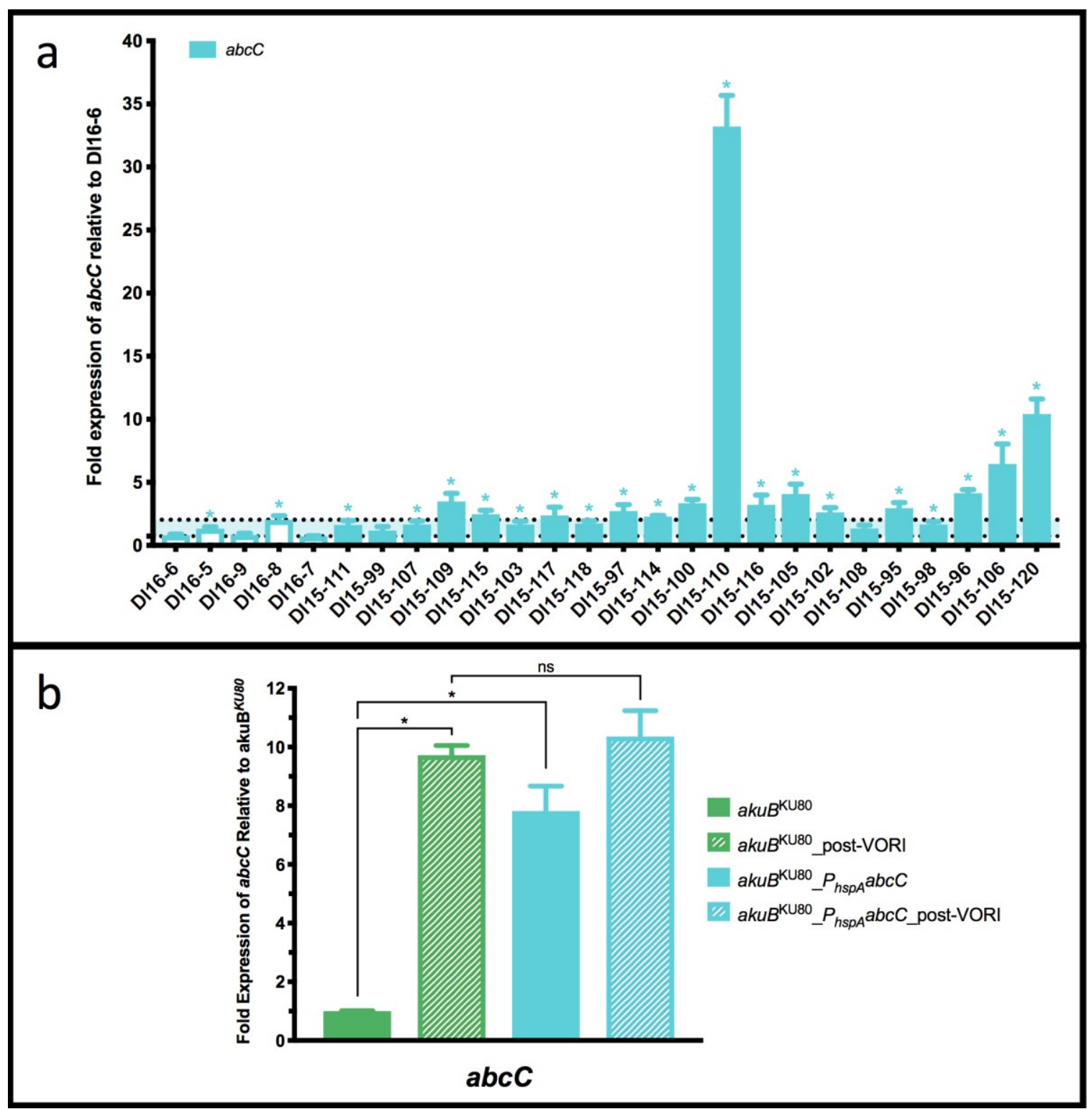

Figure 2-4. Relative expression of $a b c C$ in triazole-resistant clinical isolates and the constitutive overexpression strain $a k u B^{\mathrm{KU} 80}{ }_{-} P_{h p p A} a b c C$.

Triazole-susceptible isolates shown as open bars, triazole-resistant isolates shown as filled bars. Triazole resistant isolates shown in order of escalating voriconazole MIC from left to right. Horizontal dotted lines denote highest and lowest expression level observed among the 5 triazole-susceptible isolates. a) expression of $a b c C$ relative to triazole-susceptible isolate DI16-6. b) The expression of $a b c C$ in strains $a k u B^{\mathrm{KU} 80}$ and $a k u B^{\mathrm{KU} 80}{ }_{\text {hsp } A} a b c C$ was determined by RTqPCR following growth in Aspergillus minimal media at $37^{\circ} \mathrm{C}$ both with voriconazole $(0.125 \mathrm{mg} / \mathrm{L})$ treatment for 6 hours, and without voriconazole treatment. The expression level for each sample is shown relative to that of the respective gene in $a k u B^{\mathrm{KU} 80}$ without voriconazole treatment. Comparisons with statistically significant $(\mathrm{p}<0.05)$ results are noted with an Asterix, while those that are not are noted with "ns". 
parental strain $a k u B^{\mathrm{KU} 80}$ was assessed by RTqPCR following growth in Aspergillus minimal media at $37{ }^{\circ} \mathrm{C}$ both with voriconazole treatment $(0.125 \mathrm{mg} / \mathrm{L})$ for 6 hours and without.

In the parental $a k u B^{\mathrm{KU} 80}$ strain, $a b c C$ expression was significantly (9.7-fold) higher following voriconazole treatment than was observed without voriconazole treatment (Figure 2-4B). By comparison, the $a k u B^{\mathrm{KU} 80} P_{h s p A} a b c C$ strain exhibited 7.8fold and 10.4-fold $a b c C$ expression, under untreated and voriconazole-treated conditions, respectively, as compared to the expression of $a b c C$ measured in the parental $a k u B^{\mathrm{KU} 80}$ under untreated conditions. $a b c C$ expression was not found to be significantly different between $a k u B^{\mathrm{KU} 80}{ }_{2}{ }_{h s p A} a b c C$ and the parental strain when both had been treated with voriconazole for 6 hours. Surprisingly, even with an approximate 10 -fold increase in the constitutive expression of $a b c C$, the $a k u B^{\mathrm{KU} 80} P_{h s p A} a b c C$ strain did not demonstrate any change in voriconazole susceptibility relative to the parental $a k u B^{\mathrm{KU} 80}$ strain (MIC $0.25 \mathrm{mg} / \mathrm{L})$.

\section{Conclusions}

In this work, we performed a comprehensive characterization of the direct contributions of previously identified mechanisms of triazole resistance in a collection of triazole-resistant clinical isolates of A. fumigatus. We observed that correction of cyp $51 \mathrm{~A}$ mutations in 10 pan-triazole-resistant clinical isolates only restored wild-type susceptibility, to even a single triazole agent, in 3 isolates. We then demonstrated that while constitutive overexpression of cyp $51 \mathrm{~A}$, and to a lesser extent cyp51B, was common among isolates in this collection, increasing expression of either sterol-demethylase gene by as much as 18 to 36 -fold in the triazole-susceptible $a k u B^{\mathrm{KU} 80}$ background, had a limited effect on triazole MIC. Analogously, we then observed that while the expression of the ABC-type efflux pump-encoding gene $a b c C$ was higher among 14 of the triazoleresistant isolates in this collection, constitutive overexpression of this gene in the $a k u B^{\mathrm{KU} 80}$ background did not have any effect on voriconazole MIC.

Taken together, these data serve to demonstrate that while the canonical mechanisms of triazole resistance, even when considered collectively, do not adequately explain the triazole resistance observed in this collection of A. fumigatus clinical isolates, and additional mechanisms yet to be identified are likely present in this collection. 


\section{CHAPTER 3. THE ASPERGILLUS FUMIGATUS EFFLUX PUMP-ENCODING GENES $A B C A$ AND $A T R I$ ARE NOT SIGNIFICANT CONTRIBUTORS TO CLINICAL TRIAZOLE RESISTANCE*}

\section{Introduction}

Aspergillus fumigatus is the principal etiological agent of invasive aspergillosis, a devastating disease state responsible for more than 200,000 life-threatening infections each year, and predominantly afflicting the expanding population of immunocompromised patients.[1,2] Unfortunately, even among patients appropriately diagnosed and receiving the best available treatment, clinical outcomes associated with invasive aspergillosis remain poor with mortality rates ranging from 30-90\%.[3-5, 60]

Potentially contributing to these poor outcomes, is the paucity of therapeutic options for the treatment of invasive aspergillosis. Of the relatively limited armamentarium of clinically available antifungals, only the triazoles, the echinocandins, and the polyene amphotericin $\mathrm{B}$, are commonly utilized for the treatment of invasive aspergillosis. However, amphotericin B is most often recommended as salvage or secondline therapy, because of both limitations in available formulations and a less favorable safety profile, and the echinocandins are most frequently used as adjunctive therapy due to a lack of fungicidal activity against Aspergillus.[10] Thus, the anti-Aspergillus triazoles, including voriconazole, isavuconazole, itraconazole, and posaconazole, have proven essential to the treatment of invasive aspergillosis, and are commonly relied upon for both front-line and salvage therapies. Yet, the emergence isolates of Aspergillus fumigatus from both clinical and environmental samples, represents a critical treat to the future utility of this crucial class of anti-Aspergillus agents. [5, 6, 8, 37, 52]

Over the last several years, the identification of triazole-resistant $A$. fumigatus has been increasingly reported worldwide. In certain regions such as northern Europe, where the rate of triazole-resistant $A$. fumigatus had previously approximated the global average of $5 \%$, resistance rates exceeding $15 \%$ are now being reported. [7, 51,55] While a growing body of evidence supports the agricultural use of antifungals as a significant contributor to this emergence of resistance, the underlying molecular mechanisms of triazole resistance are less well understood.[6, 76-78] The majority of the molecular mechanisms of triazole resistance which have been characterized to date involve the genes encoding sterol-demethylase, the enzymes which the triazoles competitively inhibit.[79] A number of mutations encoding amino acid substitutions in one of the $A$. fumigatus sterol-demethylase enzymes, Cyp51A, are known to decrease triazole susceptibility when introduced into susceptible laboratory strains of A. fumigatus.[79]

\footnotetext{
* Modified from final submission with permission. Rybak JM, Ge W, Wiederhold NP, Parker JE, Kelly SL, Rogers PD and Fortwendel JR. Mutations in hmgl, challenging the paradigm of clinical triazole resistance in Aspergillus fumigatus. MBio. 2019 Apr 2;10(2). pii: e00437-19. doi: 10.1128/mBio.00437-19
} 
Additionally, mutations altering both cis- and trans-regulatory elements controlling the expression of cyp51A have also been shown to contribute to triazole resistance. However, in our previous studies we have demonstrated that mutations in cyp $51 \mathrm{~A}$ and overexpression of sterol demethylase genes cannot explain the high level of triazole resistance observed among many clinical isolates, suggesting the contribution of additional mechanisms not yet identified.

Another common mechanism of triazole antifungal resistance in pathogenic fungi is the up-regulation of efflux pump-encoding gene expression. The A. fumigatus genome is predicted to contain more than 300 efflux pump-encoding genes, many of which have been observed to have their expression induced by exposure to the triazoles.[25, 42-44, 66] Furthermore, a number of these transporters have been observed to be constitutively overexpressed among triazole resistant clinical isolates.[25] In triazole-susceptible laboratory strains of $A$. fumigatus, the individual disruption of six efflux pump genes $(a b c A, a b c C, a t r F, a t r I, m d r 1$, and $m d r A$ ) has been observed to increase triazole susceptibility. [25, 42-44, 66] However, only the small molecule transporter gene, $a b c A$, has been demonstrated to contribute to triazole resistance when constitutively overexpressed in a triazole-susceptible laboratory strain of A. fumigatus. Additionally, $a b c C$ (also called $c d r 1 B, a b c B$, and $a b c G 1$ ) is the only transporter gene which has been demonstrated to contribute to clinical triazole resistance. Disruption of $a b c C$ in a triazoleresistant clinical isolate of $A$. fumigatus decreased the itraconazole minimum inhibitory concentration (MIC) by greater than 4-fold, while voriconazole and posaconazole susceptibility remained unchanged. This clinical isolate was observed to exhibit increased constitutive expression of $a b c C$ ( $>5$ fold) relative to the susceptible reference strain. Efflux pumps have therefore been proven to be a mechanism by which triazole resistance is mediated in clinical isolates of A. fumigatus. However, the extent of the contribution of overexpression of these transporters to clinical triazole resistance is not known.

In this work, we identify the A. fumigatus efflux pumps with the highest degree of homology to $C$. albicans Cdr1, a well characterized efflux pump known to significantly contribute to clinical triazole resistance.[80, 81] We then perform transcriptional profiling on a collection of twenty-one highly triazole-resistant clinical isolates of A. fumigatus as well as five triazole-susceptible control isolates to identify which of the genes encoding the identified efflux pumps are differentially expressed among triazole-resistant isolates. Of these genes, $a b c A$ and atrI were identified as the candidates of greatest interest, and selected for deletion in clinical isolates observed to constitutively overexpress these genes using a Cas9-mediated gene transformation system. We subsequently demonstrate that the deletion of either $a b c A$ or atrI in highly triazole resistant clinical isolates of $A$. fumigatus known to overexpress these efflux pump-encoding genes, is insufficient to significantly restore triazole susceptibility. 


\section{Materials and Methods}

\section{Isolates, Media, and Growth Conditions Used}

Twenty-one previously characterized multi-triazole-resistant clinical isolates of $A$. fumigatus, and five triazole-susceptible control clinical isolates of A. fumigatus, originating from the United States were obtained from the Fungus Testing Laboratory at the University of Texas in San Antonio.[8] All clinical isolates were maintained on glucose minimal media (GMM) agar at $37^{\circ} \mathrm{C}$. Transformants were selected for using sorbitol (1.2M) supplemented GMM agar (SMM) containing $150 \mathrm{mg} / \mathrm{L}$ of hygromycin. All conidia were harvested in sterile water from three-day-old growth plates and conidia were enumerated visually using a hemocytometer.

\section{Transcriptional Profiling}

For assessment of the expression of efflux pump-encoding genes of interest in clinical isolates, conidia from each isolate were allowed to germinate overnight in Aspergillus minimal media incubated at $37^{\circ} \mathrm{C}$ on an orbital shaker at $250 \mathrm{RPM}$ in biological triplicate. Then RNA was isolated by TriZol based extraction from mature hyphae following liquid nitrogen crush as previously described.[68] RNA samples were purified using the Qiagen ${ }^{\circledR}$ RNeasy kit. Barcoded libraries were prepared and sequenced on the Illumina MiSeq platform. Bowtie2 was utilized to create the A. fumigatus Af293 reference genome index, followed by alignment of RNAseq reads to the reference genome by TopHat2. Transcript assemblies will then be generated from the aligned RNAseq reads by Cufflinks, and Cuffmerge was utilized to create a single annotated transcriptome. Transcript counts from each sample, normalized by reads per kilobase of exon length per million mapped reads, were then compared to the triazole susceptible control clinical isolate DI-16-6 (voriconazole MIC $0.25 \mathrm{mg} / \mathrm{L}$ ) to obtain fold change values. Statistical analysis was performed using unpaired, two-tailed, students t-test in Prism 8 for Mac OS by GraphPad Software Inc. with significance set at 0.05 and degrees of freedom equal to 10 .

\section{Construction of Efflux Pump-Encoding Gene Deletion Repair Templates}

Repair templates consisting of the resistance marker $(h p h R)$ and 50 bases of microhomology targeting the genes of interest were prepared by PCR as previously described.[67] Briefly, the hygromycin B resistance cassette, including the $g d p A$ promoter and $h p h R$ gene was amplified by PCR from the pUCGH plasmid using primers that introduced approximately 50 bases of homology with the regions immediately upstream and downstream of either the $a b c A$ or $\operatorname{atrI}$ open reading frame. Subsequently, the Gene Clean II Kit (MP Biomedicals) was used to purify PCR products following confirmation of product size by gel electrophoresis. Cas9-RNP complexes targeting sequences immediately upstream and downstream of the open reading frame of $a b c A$ and 
atrI were assembled as previously described.[67] Target specific primers and guide sequences are listed in Table 3-1.

\section{Aspergillus Protoplast Transformations}

Transformation of $A$. fumigatus protoplasts were performed as previously described with minor modifications.[67] Approximately $2 \mu \mathrm{g}$ of each portion applicable transformation repair templates were then mixed with $200 \mu \mathrm{L}$ of protoplasts, $26.5 \mu \mathrm{L}$ of Cas9-RNP complexes, and $25 \mu \mathrm{L}$ of polyethylene glycol (PEG)-CaCl2 buffer ( $60 \%$ [wt/vol] PEG 3350, $50 \mathrm{mM} \mathrm{CaCl} 2 \cdot \mathrm{H} 2 \mathrm{O}, 450 \mathrm{mM}$ Tris-HCl, $\mathrm{pH} 7.5)$ as previously described. Following an incubation on ice for approximately one hour, $1.25 \mathrm{~mL}$ PEG$\mathrm{CaCl} 2$ was added and the mixture incubated for an additional 20 minutes at room temperature. The mixture was then diluted to a total volume of $3 \mathrm{~mL}$ with STC buffer (1.2 $\mathrm{M}$ sorbitol, $7.55 \mathrm{mM} \mathrm{CaCl} 2 \cdot \mathrm{H} 2 \mathrm{O}, 10 \mathrm{mM}$ Tris- $\mathrm{HCl}, \mathrm{pH} 7.5)$ and plated on SMM agar plates. Plates were then incubated overnight prior to being overlaid with SMM top agar (GMM supplemented with 1.2 M sorbitol and 0.7\% [wt/vol] agar) supplemented with hygromycin (final concentration of $150 \mathrm{~g} / \mathrm{ml}$ ), and the plates were incubated at $37^{\circ} \mathrm{C}$ for 3 days. Transformants were isolated to separate plates containing selective agar, genomic DNA extracted, and successful CRISPR/Cas9 editing confirmed by PCR screens using gene-specific primers.

\section{Clinical Antifungal Susceptibility Testing}

Susceptibilities for voriconazole, isavuconazole, itraconazole, and posaconazole were determined for all isolates in accordance with Clinical Laboratory Standards Institute (CLSI) M38-A2 methodology utilizing broth microdilution in RPMI in technical triplicate.[69] Each antifungal was obtained from the appropriate manufacturer. All agents were suspended in dimethyl sulfoxide (DMSO) for preparation of stock solutions.

\section{Results}

\section{Analysis of the Expression of the Efflux Pump-Encoding Genes $a b c A$ and $a t r I$ Among Triazole-Resistant Clinical Isolates}

To identify candidate efflux-pumps encoding genes which may contribute to triazole resistance in clinical isolates of $\mathrm{A}$. fumigatus, the genes in the reference Af $293 \mathrm{~A}$. fumigatus genome with a predicted peptide sequences possessing the highest homology to C. albicans Cdr1 were identified by BLASTP. Twelve genes (Table 3-2) were identified to have markedly higher homology than any other gene (BLAST P scores greater than 800 , E values of 0.0 , and greater than $35 \%$ matching residue identities). Of these, four have been previously associated with triazole resistance in A. fumigatus 
Table 3-1. Oligonucleotides used for $a b c A$ and atrI experiments.

\begin{tabular}{|c|c|c|c|}
\hline Oligo Type & Gene & Primer Name & Sequence $\left(5^{\prime}-3^{\prime}\right)$ \\
\hline \multicolumn{4}{|l|}{ PCR Primers } \\
\hline & \multirow[t]{2}{*}{$a b c A$} & \multirow[t]{2}{*}{$a b c A$ KO F CRISPR } & GTATTTCTTCCTGCCATGACCAGGCAGTCA \\
\hline & & & TCCCAGAGGTTGAGCTTGCATGCCTGCAGG \\
\hline & \multirow[t]{2}{*}{$a b c A$} & \multirow[t]{2}{*}{$a b c A$ KO R CRISPR } & CTATCAGTGCTGCAAGTCATGCAGGGTACA \\
\hline & & & TATACATCACAGCGCCCTTCCGAGCTCCC \\
\hline & \multirow[t]{2}{*}{ atrI } & \multirow{2}{*}{ atrI KO F CRISPR } & CCACTGTGGATTCTCGGCATCGTCATTCAT \\
\hline & & & TTTTCTTCCGAGCTTGCATGCCTGCAGG \\
\hline & \multirow[t]{2}{*}{ atrI } & \multirow[t]{2}{*}{ atrI KO R CRISPR } & GGCGGCAGGAAAGATGTGGATTTTCTATGT \\
\hline & & & ATAAGAATAGCGCCCTTCCGAGCTCCC \\
\hline & \multirow[t]{2}{*}{$a b c A$} & \multirow[t]{2}{*}{$a b c A$ screen $\mathrm{F}$} & GTTCGACTGTTTATGTGTATACCTATTGTG \\
\hline & & & ACGCAACCACTCAGCTTGCATGCCTGCAGG \\
\hline & \multirow[t]{2}{*}{ atrI } & \multirow{2}{*}{ atrI screen $\mathrm{F}$} & GGGTTGATGTTAGGATTTATCGTCCCTAGA \\
\hline & & & $\begin{array}{l}\text { AGAGACATGCTTGGTACCTGTGAAGAAGT } \\
\text { G }\end{array}$ \\
\hline & $h p h R$ & $h p h R$ screen $\mathrm{R}$ & CAAATCGCCCGCAGAAGCG \\
\hline \multicolumn{4}{|c|}{ crRNA Guide Sequences } \\
\hline & $a b c A$ & $a b c A 5^{\prime}$ crRNA & TGGTGTGAGTCGTGCAGCGA \\
\hline & $a b c A$ & $a b c A 3^{\prime}$ crRNA & CTTATTGCTGTTCGTCAGCT \\
\hline & atrI & atrI 5' crRNA & ATTATGGGTGTATAGATGAA \\
\hline & atrI & atrI 5' crRNA & CATGTACACACCCACTATCA \\
\hline
\end{tabular}


Table 3-2. Relative expression of efflux pump-coding genes of interest among triazole-resistant clinical isolates.

\begin{tabular}{|c|c|c|c|c|c|c|c|c|c|c|c|c|c|c|c|c|c|c|c|c|c|c|c|c|c|c|}
\hline \multirow[b]{2}{*}{ Gene (Name) } & \multicolumn{5}{|c|}{ Susceptible Isolates } & \multicolumn{21}{|c|}{ Resistant Isolates } \\
\hline & $\begin{array}{l}0 \\
b \\
= \\
\overrightarrow{0}\end{array}$ & $\begin{array}{l}n \\
\tilde{b} \\
=\vec{\sigma}\end{array}$ & $\begin{array}{l}\text { bे } \\
\stackrel{\bar{b}}{1}\end{array}$ & 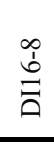 & $\begin{array}{l}\hat{b} \\
\overrightarrow{0}\end{array}$ & $\begin{array}{l}\vec{\Xi} \\
\dot{\vec{b}} \\
\vec{\Xi}\end{array}$ & 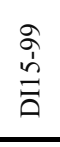 & $\begin{array}{l}\hat{\sigma} \\
\dot{n} \\
\overrightarrow{\overline{0}}\end{array}$ & $\begin{array}{l}\stackrel{\circ}{0} \\
\dot{n} \\
\stackrel{\overrightarrow{0}}{1}\end{array}$ & $\begin{array}{l}\stackrel{n}{\Xi} \\
\stackrel{\dot{n}}{\Xi}\end{array}$ & 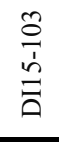 & 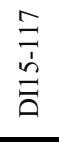 & 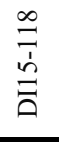 & $\begin{array}{l}\hat{a} \\
\hat{\vec{b}} \\
\hat{a}\end{array}$ & $\begin{array}{l}\underset{\Xi}{\Xi} \\
\stackrel{\vec{\Xi}}{\Xi}\end{array}$ & 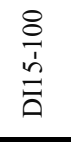 & $\begin{array}{l}\stackrel{\circ}{\vec{\prime}} \\
\stackrel{\dot{b}}{\Xi}\end{array}$ & 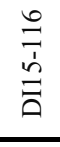 & $\begin{array}{l}n \\
o \\
\dot{a} \\
\tilde{a}\end{array}$ & 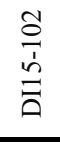 & $\begin{array}{l}\stackrel{\infty}{0} \\
\stackrel{1}{n} \\
\stackrel{\overrightarrow{0}}{1}\end{array}$ & 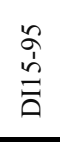 & $\begin{array}{l}\infty \\
\stackrel{1}{1} \\
\stackrel{\vec{b}}{ }\end{array}$ & 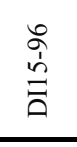 & 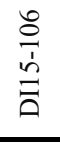 & 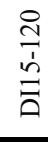 \\
\hline $\begin{array}{l}\text { Afu2g15130 } \\
(a b c A)\end{array}$ & 0.9 & 1.4 & 0.6 & $\underline{1.5}$ & 1.0 & 2.1 & 2.4 & 0.6 & 2.1 & 0.7 & 4.2 & 1.7 & 2.5 & 1.8 & 7.4 & 19.0 & 238.6 & 1.4 & 15.1 & 1.3 & 1.1 & 2.3 & 3.3 & 11.6 & 6.6 & 1.8 \\
\hline $\begin{array}{l}\text { Afu1g14330 } \\
(a b c C)\end{array}$ & 0.9 & $\underline{3.1}$ & 1.2 & 1.2 & 1.5 & 1.2 & 0.8 & 0.8 & 1.9 & 0.8 & 1.4 & 1.4 & 1.1 & 3.1 & 3.2 & 3.7 & 83.4 & 3.3 & 2.4 & 1.9 & 0.5 & 1.7 & 2.4 & 6.8 & 4.0 & 6.3 \\
\hline Afu1g17440 & $\underline{1.1}$ & 0.7 & 0.7 & 0.3 & 0.7 & 1.3 & 0.8 & 0.9 & 2.2 & 2.5 & 1.6 & 1.1 & 0.4 & 1.3 & 2.1 & 2.3 & 3.2 & 1.7 & 1.6 & 2.6 & 8.7 & 1.7 & 1.0 & 1.1 & 2.6 & 1.6 \\
\hline Afu5g00790 & 0.8 & 0.5 & 0.4 & $\underline{1.4}$ & 0.6 & 0.3 & 0.3 & 0.4 & 0.4 & 0.2 & 0.3 & 0.1 & 0.2 & 0.4 & 0.8 & 1.5 & 0.6 & 0.2 & 0.2 & 0.2 & 1.8 & 1.1 & 1.2 & 0.6 & 0.5 & 0.2 \\
\hline $\begin{array}{l}\text { Afu3g07300 } \\
\text { (atrI) }\end{array}$ & 1.1 & 0.6 & 0.8 & $\underline{1.3}$ & 0.9 & 2.0 & 1.4 & 1.3 & 1.2 & 1.7 & 0.6 & 1.5 & 1.9 & 1.6 & 2.1 & 2.2 & 8.3 & 1.8 & 2.0 & 3.5 & 1.7 & 2.0 & 2.3 & 4.6 & 8.1 & 3.3 \\
\hline Afu5g02260 & $\underline{0.9}$ & 0.6 & 0.8 & 0.7 & 0.5 & 1.5 & 0.5 & 0.5 & 0.8 & 0.3 & 0.7 & 0.6 & 0.7 & 1.5 & 0.9 & 0.9 & 1.1 & 1.0 & 0.9 & 1.1 & 1.6 & 1.3 & 1.6 & 1.3 & 1.4 & 0.6 \\
\hline Afu6g08020 & 1.1 & 1.5 & 1.3 & 1.3 & $\underline{1.6}$ & 1.8 & 1.0 & 1.0 & 3.2 & 1.5 & 5.6 & 0.9 & 2.6 & 1.3 & 1.1 & 1.9 & 3.6 & 1.3 & 22.4 & 7.9 & 1.1 & 3.3 & 3.4 & 2.4 & 6.1 & 1.4 \\
\hline Afu3g01400 & $\underline{1.1}$ & 0.5 & 0.6 & 0.5 & 0.6 & 0.7 & 0.5 & 0.4 & 0.6 & 0.6 & 0.5 & 0.6 & 0.6 & 0.7 & 0.4 & 1.3 & 2.3 & 0.7 & 0.5 & 1.4 & 1.0 & 0.7 & 0.8 & 1.8 & 1.7 & 1.0 \\
\hline $\begin{array}{l}\text { Afu6g04360 } \\
(\text { atr } F)\end{array}$ & 0.9 & 0.4 & 2.3 & $\underline{6.9}$ & 1.3 & 2.3 & 1.4 & 2.2 & 4.1 & 1.6 & 1.8 & 1.7 & 2.3 & 2.6 & 2.7 & 1.2 & 1.0 & 3.8 & 5.3 & 8.4 & 4.6 & 2.0 & 6.9 & 4.6 & 1.6 & 2.2 \\
\hline Afu4g01050 & $\underline{1.1}$ & 0.3 & 0.4 & 0.7 & 0.6 & 0.3 & 0.5 & 0.8 & 0.8 & 0.6 & 0.6 & 0.3 & 0.9 & 0.3 & 0.5 & 0.2 & 0.8 & 0.2 & 1.5 & 0.5 & 0.8 & 0.4 & 1.4 & 0.3 & 0.4 & 0.5 \\
\hline Afu5g09460 & 0.9 & 0.9 & 0.9 & $\underline{4.7}$ & 1.3 & 1.1 & 0.7 & 0.8 & 0.9 & 0.6 & 0.5 & 0.2 & 0.6 & 1.7 & 0.3 & 1.3 & 0.8 & 0.4 & 0.8 & 0.9 & 0.8 & 1.9 & 0.5 & 1.4 & 0.6 & 0.7 \\
\hline Afu8g02650 & 1.1 & 1.2 & 1.2 & 1.2 & $\underline{1.6}$ & 0.6 & 0.8 & 1.3 & 1.0 & 0.9 & 0.6 & 0.9 & 1.2 & 1.8 & 1.2 & 1.4 & 1.1 & 1.6 & 1.6 & 1.7 & 1.3 & 2.2 & 1.4 & 1.5 & 1.7 & 1.0 \\
\hline
\end{tabular}

A. fumigatus clinical isolates are ordered by escalating voriconazole MIC from left to right. A fumigatus genes are listed by degree of homology with C. albicans $\mathrm{Cdr1}$ from top to bottom. Genes shown in bold have previously been associated with triazole resistance in A. fumigatus. Fold expression values are shown as the average of three independent biological samples relative to the triazolesusceptible control isolate DI16-6. Fold expression values shown underlined are the highest for that gene among susceptible isolates. Fold expression values among resistant isolates shown in bold are at least two times higher than the highest measured value for the same gene among the triazole susceptible control isolates (underlined value). 
( $a b c A, a b c C, a t r F$, and $a t r l)$. Additionally, disruption of $a b c C$ has previously been demonstrated to increase triazole susceptibility in both susceptible laboratory strains and a triazole-resistant clinical isolate. [25, 42-44, 66]

To assess the whether the constitutive expression of each the identified efflux pump-encoding genes of interest may be greater among triazole-resistant clinical isolates of $A$. fumigatus, we obtained twenty-six clinical isolates from the Fungus Testing Laboratory in San Antonio, Texas. This collection of isolates includes five triazolesusceptible clinical isolates, and twenty-one triazole-resistant clinical isolates that have MIC for at least two triazole agents above published CLSI epidemiological cut-off values (ECV; voriconazole, isavuconazole, and itraconazole $1 \mathrm{mg} / \mathrm{L}$, posaconazole $0.25 \mathrm{mg} / \mathrm{L}) .[73,82]$ RNA was then isolated from each clinical isolate in biological triplicate following overnight growth in Aspergillus minimal media at $37^{\circ} \mathrm{C}$ on an orbital shaker at 250 RPM, and transcriptional profiling was performed. Normalized read counts were for each sample were compared to the read counts measured in the triazolesusceptible control isolate DI16-6 (voriconazole MIC $0.25 \mathrm{mg} / \mathrm{L}$ ) to generate fold change values for each of the efflux pump-encoding genes of interest (Table 3-2).

Of the twelve efflux pump-encoding genes of interest, six were observed to be expressed at least 2-fold higher in one or more triazole-resistant isolate, as compared to the triazole-susceptible isolate with the highest measured level of expression for each gene of interest ( $a b c A, a b c C$, Afu1g17440, atrI, Afu6g08020, and Afu3g01400). Ten resistant isolates were observed to overexpress more than one gene of interest, and this was more frequently observed among more highly triazole resistant clinical isolates. $a b c A$, which has been shown to increase triazole resistance when overexpressed in a triazole-susceptible laboratory strain and which has the highest degree of homology with C. albicans Cdr1, was observed to be overexpressed in eight triazole-resistant clinical isolates (3.3 to 238.6-fold that of DI16-6). $a b c C$, which has previously been shown to contribute to clinical triazole resistance in A. fumigatus, was overexpressed in three triazole-resistant clinical isolates (6.3 to 83.4-fold that of DI16-6). Afu1g17440, which has not previously been implicated in triazole resistance, was overexpressed in seven triazole-resistant clinical isolates (2.2 to 8.7-fold). atrI, which when deleted in a triazolesusceptible laboratory strain of $A$. fumigatus leads to hyper-susceptibility, was overexpressed in five triazole-resistant isolates (3.3 to 8.3-fold that of DI16-6). Afu6g08020 and Afu3g014000 have not previously been associated with triazole resistance and were overexpressed in eight (3.2 to 22.4-fold that of DI16-6) and one (2.3 fold that of DI16-6) triazole-resistant isolates, respectively. As $a b c A$ and $a t r I$ both have previously been implicated in triazole resistance, were both found to be overexpressed in multiple triazole-resistant isolates in this collection, and have not yet been demonstrated to contribute to triazole resistance among clinical isolates of $A$. fumigatus, these efflux pump-encoding genes were selected for further interrogation. 


\section{Deletion of the Efflux Pump-Encoding Genes $a b c A$ and $a t r I$ Has Minimal Impact on Clinical Triazole Resistance}

Following the identification of $a b c A$ and $a t r I$ as the efflux pump-encoding genes of greatest interest, we sought to characterize the direct impact of these two genes on triazole resistance in clinical isolates of $A$. fumigatus. To accomplish this, we utilized a Cas9-mediated transformation system to delete $a b c A$ and $a t r I$ in triazole-resistant clinical isolates observed to exhibit constitutive overexpression of each of these genes.[67] As eight resistant clinical isolates were observed to exhibit overexpression of $a b c A$ (at least 2 -fold above the highest expression among triazole-susceptible isolates), and seven of these were found to be statistically significant (as compared to DI16-6; Figure 3-1A), we selected both DI15-100 (19-fold the $a b c A$ expression of DI16-6) and DI15-106 (6.6-fold the $a b c A$ expression of DI16-6) for deletion of $a b c A$. Importantly, DI15-110 (238.6-fold the $a b c A$ expression of DI16-6) was not selected as this isolate exhibits a slow growth rate, altered growth morphology, and diminished conidiation, all of which make genetic manipulations technically challenging. Of the five triazole-resistant isolates observed to overexpress atrI (at least 2-fold above the highest expression among triazole-susceptible isolates), all of which were statistically significant as compared to DI16-6 (Figure 3-1B), DI15-106 (8.1-fold the atrI expression of DI16-6) was selected for deletion of atrI.

Subsequently, two independent, positive transformants were constructed for each gene of interest as previously described.[67] Triazole MIC for all clinically available agents were then determined following CLSI methods for the parental triazole-resistant clinical isolates and all efflux pump-deletion strains.[69]

The voriconazole, isavuconazole, itraconazole, and posaconazole MIC for DI15100, DI-106, and all derivative efflux pump deletion strains are shown in Table 3-3. For both triazole-resistant clinical isolates, the MIC for voriconazole, isavuconazole, and itraconazole were greater than previously published ECV. Notably, the voriconazole and isavuconazole MIC for DI15-106 were above the CLSI recommended testing range $(0.03$ to $16 \mathrm{mg} / \mathrm{L})$ and at least 32-fold above the ECV $(1 \mathrm{mg} / \mathrm{L})$ for these agents. [69] Deletion of $a b c A$ in either the DI5-100 or DI15-106 clinical isolate backgrounds had no impact on isavuconazole or posaconazole MIC. In the $a b c A$ deletion strains constructed in the DI15100 background (DI15-100_abcA_A and DI15-100_abcA_B), voriconazole MIC were reduced by one dilution ( 8 to $4 \mathrm{mg} / \mathrm{L}$ ). In the $a b c A$ deletion strains constructed in the DI15-106 background (DI15-106_abcA_A and DI15-106_abcA_B), itraconazole MIC were reduced by one dilution $(2$ to $1 \mathrm{mg} / \overline{\mathrm{L}})$. While these changes in triazole MIC were reproducible, they are also within the accepted assay error range.[69] Similarly, MIC for voriconazole, isavuconazole, and itraconazole were unchanged upon deletion of atrI in the DI15-106 background. Posaconazole MIC were observed to increase by one dilution (from 0.125 to $0.25 \mathrm{mg} / \mathrm{L}$ ). 
Figure 3-1. Relative expression of $a b c A$ and $a t r I$ among triazole-resistant clinical isolates.

A) Expression of $a b c A$ in clinical isolates relative to triazole-susceptible isolate DI16-6 B) expression of atrI in clinical isolates relative to triazole-susceptible isolate DI16-6; Triazole-susceptible isolates shown as open bars, triazole-resistant isolates shown as filled bars. Triazole-resistant isolates shown in order of escalating voriconazole MIC from left to right. Horizontal dotted lines denote highest and lowest expression level observed among the five triazole-susceptible isolates. Comparisons with the susceptible control isolate DI16-6 with statistically significant $(\mathrm{p}<0.05)$ results are noted with an Asterix. 

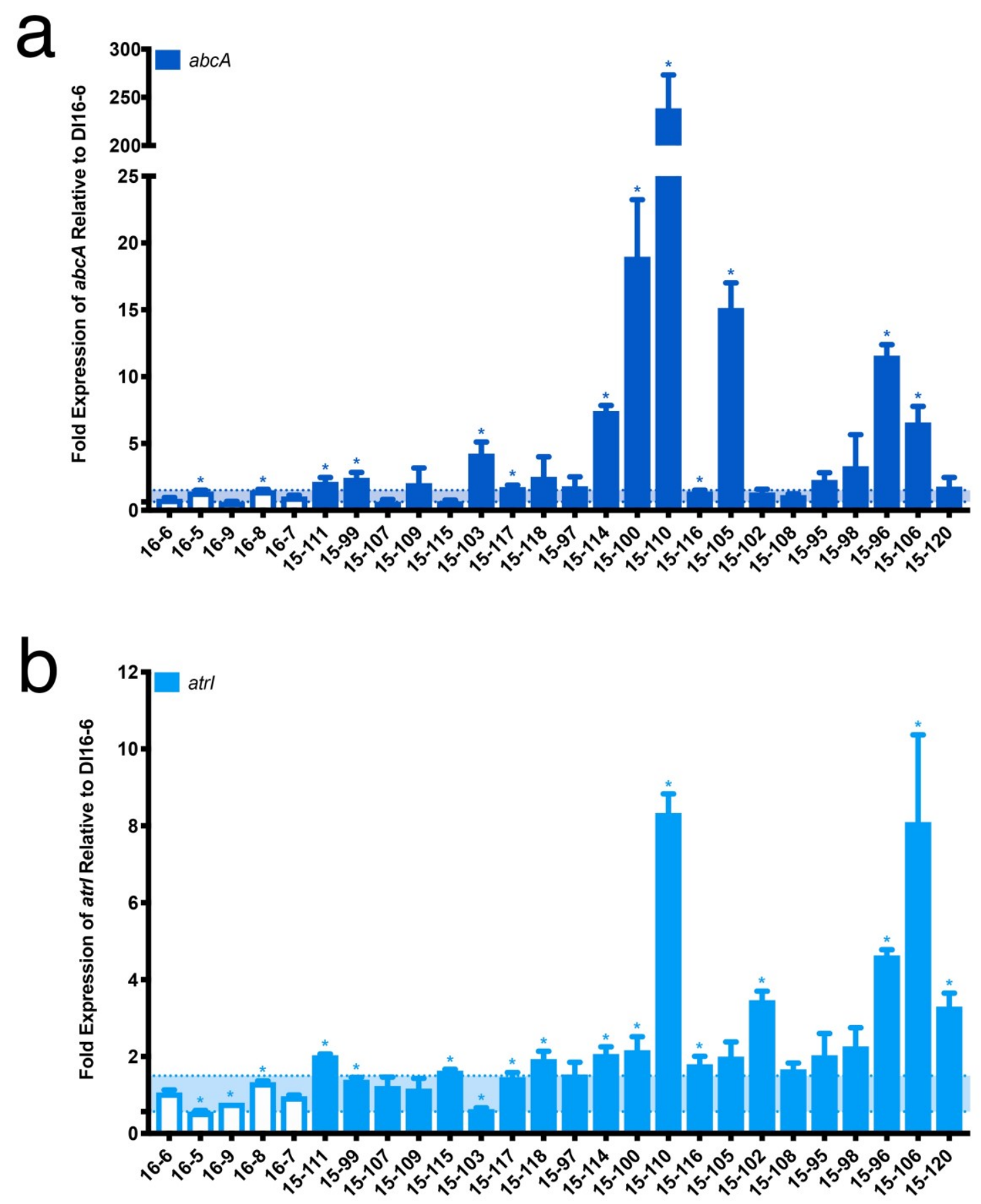
Table 3-3. Triazole MIC of clinical isolates and derivative efflux pump-encoding gene deletion strains.

\begin{tabular}{lcccc}
\hline Parent Clinical Isolate or & \multicolumn{5}{c}{ Triazole MIC (mg/L) } \\
\cline { 2 - 5 } Derivative Strain & Voriconazole & Isavuconazole & Itraconazole & Posaconazole \\
\hline DI15-100 & $\mathbf{8}$ & $\mathbf{4}$ & $\mathbf{4}$ & 0.125 \\
DI15-100_abcA_A & $\mathbf{4}$ & $\mathbf{4}$ & $\mathbf{4}$ & 0.125 \\
DI15-100_abcA_B & $\mathbf{4}$ & $\mathbf{4}$ & $\mathbf{4}$ & 0.125 \\
& & & & \\
DI15-106 & $\mathbf{3 2}$ & $\mathbf{3 2}$ & $\mathbf{2}$ & 0.125 \\
DI15-106_abcA_A & $\mathbf{3 2}$ & $\mathbf{3 2}$ & 1 & 0.125 \\
DI15-106_abcA_B & $\mathbf{3 2}$ & $\mathbf{3 2}$ & 1 & 0.125 \\
DI15-106_atrI_A & $\mathbf{3 2}$ & $\mathbf{3 2}$ & $\mathbf{2}$ & 0.25 \\
DI15-106_atrI_B & $\geq \mathbf{3 2}$ & $\mathbf{3 2}$ & $\mathbf{2}$ & 0.25 \\
\hline
\end{tabular}

MIC shown in bold are greater than epidemiologic cutoff values as published by the Clinical Laboratory and Standards Institute $(1 \mathrm{mg} / \mathrm{L}$ for voriconazole and itraconazole; $0.25 \mathrm{mg} / \mathrm{L}$ for posaconazole). 


\section{Conclusions}

In this work we attempted to identify efflux pump-encoding genes which may contribute to triazole resistance in clinical isolates of $A$. fumigatus. We identified twelve efflux pump encoding genes in the Af293 reference $A$. fumigatus genome with predicted peptides sequences that have the highest degree of homology with the well characterized C. albicans triazole efflux pump Cdr1. Importantly, four of the identified genes of interest have previously been associated with triazole resistance, and one of these, $a b c C$, has been demonstrated to directly contribute to clinical triazole resistance. [25, 42-44, 66] The remaining eight genes have not previously been well characterized.

We then performed transcriptional profiling on a collection of twenty-one multitriazole-resistant clinical isolates of A. fumigatus, as well as five triazole-susceptible control clinical $A$. fumigatus isolates, to identify which of these efflux pump-encoding genes are constitutively overexpressed among triazole-resistant clinical isolates. Transcriptional profiling revealed that six of the twelve efflux pump-encoding genes of interest were more highly expressed among triazole resistant isolates. These six genes included $a b c A, a b c C$, and $a t r I$, which have previously been implicated in triazole resistance, as well as Afu1g17440, Afu6g08020, and Afu3g01400 which have not. Interestingly, ten triazole-resistant isolates were observed to overexpress two or more genes of interest, and this was observed more frequently among isolates which are more highly triazole-resistant. Additionally, atr $F$, which has also previously been implicated in triazole resistance, was not observed to be more highly expressed among the triazoleresistant isolates in this collection.

We subsequently identified both $a b c A$ and $a t r I$ as the genes of greatest interest for further investigation, due to both the observed degree of overexpression among triazoleresistant isolates in our collection, and the findings of previous studies implicating these genes in clinical triazole resistance. Surprisingly, upon deletion of $a b c A$ in both the DI15100 and DI15-106 triazole-resistant clinical isolate backgrounds, no pronounced change in triazole susceptibility was observed. Similarly, when atrI was deleted in the DI15-106 background, no decrease in triazole resistance occurred. Yet, it remains possible that that these highly triazole-resistant clinical isolates harbor multiple triazole resistance mechanisms, such as mutations in the sterol-demethylase gene cyp51A, which may mask smaller changes in triazole susceptibility upon deletion of the efflux pump encoding genes of interest. In fact, in previous studies we have shown that DI15-100 and DI15-106 both harbor mutations in cyp51A (encoding M263I and TR $46 / \mathrm{Y} 121 \mathrm{~F} / \mathrm{T} 289 \mathrm{~A}$, respectively). However, we have also demonstrated that correction of these cyp51A mutations in DI15-100 and DI15-106 also failed to restore the MIC of any triazole agent to wild-type susceptible ranges.

Taken together, the findings of this study serve to demonstrate that A. fumigatus efflux pump-encoding genes with homology to known triazole resistance genes are constitutively overexpressed among triazole-resistant clinical isolates. However, deletion of either $a b c A$ or $a t r I$ in resistant clinical isolates which constitutively overexpress them was insufficient to significantly restore triazole susceptibility. Future studies are needed 
to further characterize the potential role of these and other A. fumigatus efflux pumpencoding genes in clinical triazole resistance, the genetic determinants which lead to increased efflux pump gene expression among triazole-resistant clinical isolates of $A$. fumigatus. 


\title{
CHAPTER 4. MUTATIONS IN THE ASPERGILLUS FUMIGATUS HMG-COA REDUCTASE GENE, HMG1, A NOVEL GENETIC DETERMINANT OF CLINICAL TRIAZOLE RESISTANCE*
}

\begin{abstract}
Introduction
The most common invasive mold infection is invasive aspergillosis, with Aspergillus fumigatus being identified as the etiological agent for more than $72 \%$ of all cases.[2] Worldwide invasive aspergillosis is implicated in more than 200,000 lifethreatening infections each year, and is estimated to affect more than $10 \%$ of all patients with acute leukemia or having received bone marrow or other transplants. [1] While substantial healthcare resources are allocated to the treatment of invasive aspergillosis, rates of clinical failure remain unacceptably high, approximating 40\%.[4, 83, 84]

The triazole class of antifungals are essential to the treatment of invasive aspergillosis. Routinely relied upon as both front-line (voriconazole and isavuconazole) and salvage (itraconazole and posaconazole) therapeutic options, these agents are available in a range of oral and intravenous formulations and generally are well tolerated.[10, 13, 79] The triazoles exert fungicidal activity against Aspergillus through the inhibition of the biosynthesis of ergosterol, and essential constituent of fungal cell membranes.[21] The triazoles impede ergosterol biosynthesis through competitive inhibition of the sterol-demethylase proteins, Cyp51A and Cyp51B. Inhibition of these two enzymes ultimately leads to a depletion of cellular ergosterol and the accumulation of methylated ergosterol precursors.[14] Unfortunately, the treatment of invasive aspergillosis has been further complicated by the emergence of $A$. fumigatus isolates resistant to the triazoles. While the rate of triazole resistance in the United States is currently estimated to be $5 \%$, globally reports of triazole-resistant $A$. fumigatus clinical isolates are increasing. In countries such as the United Kingdom and the Netherlands, where triazole resistance rates were previously comparable to those in the United States, resistance rates now exceed $15 \%$.[6-8, 30]
\end{abstract}

A limited number of triazole resistance mechanisms have been identified in $A$. fumigatus, largely mirroring mechanisms of triazole resistance previously identified in other pathogenic fungi such as Candida albicans. Single nucleotide polymorphisms (SNPs) resulting in amino acid substitutions in one of the two sterol-demethylase enzymes, Cyp51A, overexpression of the gene encoding this sterol-demethylase enzyme, cyp $51 \mathrm{~A}$, and overexpression of the efflux pump-encoding gene, $a b c C$, have all been shown to contribute to triazole resistance among clinical isolates of $A$. fumigatus. [25, 28, $34,35,42-44,48,66,71,79]$ However, we have previously demonstrated that these

* Modified from final submission with permission. Rybak JM, Ge W, Wiederhold NP, Parker JE, Kelly SL, Rogers PD and Fortwendel JR. Mutations in hmgl, challenging the paradigm of clinical triazole resistance in Aspergillus fumigatus. MBio. 2019 Apr 2;10(2). pii: e00437-19. doi: 10.1128/mBio.00437-19 
mechanisms alone poorly explain the high level of triazole resistance observed among many clinical isolates of $A$. fumigatus. Thus, the molecular mechanisms underpinning much of the clinical resistance to this essential class of anti-Aspergillus agents remains unexplained, limiting the discovery of therapeutic strategies to overcome triazoleresistant aspergillosis.

We describe here for the first time a novel mechanism of triazole resistance, not mediated by mutations in sterol-demethylase and never before observed among pathogenic fungi, which was found to be present in a majority of triazole-resistant clinical isolates of $A$. fumigatus in our collection. Mutations in the 3-hydroxy-3-methyl-glutarylcoenzyme A (HMG-CoA) reductase encoding gene, hmgl (Afu2g03700), were found to result in dramatically increased resistance to all clinically available triazole agents. Additionally, the restoration of $\mathrm{hmgl}$ to the wild-type sequence in a clinical A. fumigatus isolate exhibiting high-level pan-triazole resistance was found to restore clinical susceptibility to the triazole class. We demonstrate that mutations in $\mathrm{hmgl}$ result in maintained or even increased ergosterol production as well as accumulation of multiple ergosterol precursors, including eburicol, the substrate of the sterol-demethylase enzymes which the triazole antifungals competitively inhibit.

\section{Materials and Methods}

\section{Isolates, Media, and Growth Conditions Used}

Twenty-one previously characterized multi-triazole-resistant clinical isolates of $A$. fumigatus, and five triazole-susceptible control clinical isolates of A. fumigatus, originating from the United States were obtained from the Fungus Testing Laboratory at the University of Texas in San Antonio.[8] The laboratory strains $a k u B^{\mathrm{KU} 80}$ and Af293 were obtained from the Fungal Genetics Stock Center. All strains and clinical isolates used in this study were maintained on glucose minimal media (GMM) agar at $37^{\circ} \mathrm{C}$. Transformants were selected for using sorbitol (1.2M) supplemented GMM agar (SMM) containing $150 \mathrm{mg} / \mathrm{L}$ of hygromycin. All conidia were harvested in sterile water from three-day-old growth plates and conidia were enumerated visually using a hemocytometer.

\section{Construction of Allele Replacement Repair Templates and Cas9-RNP for the hmg1 Locus}

Two component repair templates consisting of a split hygromycin resistance marker $(h p h R)$ and $h m g l$ alleles of interest were prepared by PCR. Briefly, $h m g l$ alleles of interest including the open reading frame and approximately 500 downstream bases were amplified by PCR from DI15-98 (S305P), DI15-100 (I412S), DI15-105 (F262del), and akuBKU80 (wild-type control) using a 3' primer which introduced the terminal 80 bases with homology to the 3' end of the $h p h R$ hygromycin B resistance gene open 
reading frame. A partial hygromycin $\mathrm{B}$ resistance cassette, including the $g d p A$ promoter and a truncated $h p h R$ gene lacking the terminal 40 bases, was then amplified by PCR from the pUCGH plasmid using primers that introduced approximately 70 bases of homology with the downstream region of $h m g l$. PCR products were subsequently purified using the Gene Clean II Kit (MP Biomedicals). Cas9-RNP complexes targeting sequences immediately upstream and approximately 500 bases downstream of the open reading frame of hmg1 were assembled as previously described.[67] Target specific guide sequences and primers are listed in Table 4-1.

\section{Aspergillus Protoplast Transformations}

Transformation of $A$. fumigatus protoplasts were performed as previously described with minor modifications.[67] Approximately $2 \mu \mathrm{g}$ of each portion applicable transformation repair templates were then mixed with $200 \mu \mathrm{L}$ of protoplasts, $26.5 \mu \mathrm{L}$ of Cas9-RNP complexes, and $25 \mu \mathrm{L}$ of polyethylene glycol (PEG)-CaCl2 buffer $(60 \%$ [wt/vol] PEG 3350, $50 \mathrm{mM} \mathrm{CaCl2} \cdot \mathrm{H} 2 \mathrm{O}, 450 \mathrm{mM}$ Tris-HCl, pH 7.5) as previously described. Following an incubation on ice for approximately one hour, $1.25 \mathrm{~mL}$ PEG$\mathrm{CaCl} 2$ was added and the mixture incubated for an additional 20 minutes at room temperature. The mixture was then diluted to a total volume of $3 \mathrm{~mL}$ with STC buffer (1.2 M sorbitol, $7.55 \mathrm{mM} \mathrm{CaCl} \cdot \mathrm{H} 2 \mathrm{O}, 10 \mathrm{mM}$ Tris-HCl, $\mathrm{pH} 7.5$ ) and plated on SMM agar plates. Plates were then incubated overnight prior to being overlaid with SMM top agar (GMM supplemented with 1.2 M sorbitol and 0.7\% [wt/vol] agar) supplemented with hygromycin (final concentration of $150 \mathrm{~g} / \mathrm{ml}$ ), and the plates were incubated at $37^{\circ} \mathrm{C}$ for 3 days. Transformants were isolated to separate plates containing selective agar, genomic DNA extracted, and successful CRISPR/Cas9 editing confirmed by PCR screens using gene-specific primers and Sanger sequencing.

\section{Clinical Antifungal Susceptibility Testing}

Susceptibilities for amphotericin B, voriconazole, isavuconazole, itraconazole, and posaconazole were determined for all isolates in accordance with CLSI M38-A2 methodology utilizing broth microdilution in RPMI.[69] Each antifungal was obtained from the appropriate manufacturer. All agents were suspended in dimethyl sulfoxide (DMSO) for preparation of stock solutions.

\section{Comprehensive Sterol Profiling.}

Fresh conidial suspensions of each strain to be studied were prepared in saline with tween 80 from AMM agar plates. Conidia were grown in 25mL RPMI supplemented with $0.2 \%$ glucose buffered to $\mathrm{pH} 7.0$ with MOPS for 24 hours in an orbital shaker at 180 RPM and $35^{\circ} \mathrm{C}$. Cells were then flash frozen using liquid nitrogen, dry weights obtained, and alcoholic $\mathrm{KOH}$ was utilized to extract nonsaponifiable lipids. A vacuum centrifuge (Heto) was then used to dry samples, prior to being derivatized by the addition of $200 \mu \mathrm{L}$ 
Table 4-1. Oligonucleotides used for $h m g 1$, cyp51A, and cyp51B experiments.

\begin{tabular}{|c|c|c|c|}
\hline Oligo Type & Gene & Primer Name & Sequence (5'- 3') \\
\hline \multicolumn{4}{|l|}{ PCR Primers } \\
\hline & \multirow[t]{3}{*}{ hmgl } & \multirow[t]{3}{*}{$h m g l \mathrm{~F}$} & GACAGGCGGCTTACACCGCCTCTCCTGCT \\
\hline & & & CGCCATTTTGTCTATGATCACTGATGGCT \\
\hline & & & \\
\hline & \multirow[t]{4}{*}{ hmgl } & \multirow[t]{4}{*}{$h m g 1$ split $h p h R \mathrm{R}$} & TCTGGACCGATGGCTGTGTAGAAGTACT \\
\hline & & & CGCCGATAGTGGAAACCGACGCCCCAGC \\
\hline & & & ACTCGTCCGAGGGCAAAGGAATAGGCTG \\
\hline & & & CGTTACTCGGTCTTGG \\
\hline & $h p h R$ & $h p h R$ split $\mathrm{F}$ & CCACTATCGGCGAGTACTTCTACAC \\
\hline & \multirow[t]{4}{*}{$h p h R$} & \multirow[t]{4}{*}{$h p h R$ 3'UTR hmgl R } & CCCACCTGGGATGAAGCAAAAGCGGGTA \\
\hline & & & CATAGATAGGTTTATGGGGACTTTGGGA \\
\hline & & & AGTGAAATATGGGCGAGCTCCCAAATCT \\
\hline & & & GTCCAG \\
\hline & hmgl & $h m g 1$ screen F & GGTGTCCGTGGTTCCCACC \\
\hline & $h p h R$ & $h p h R$ screen $\mathrm{R}$ & CAAATCGCCCGCAGAAGCG \\
\hline \multicolumn{4}{|c|}{ crRNA Guide Sequences } \\
\hline & $h m g 1$ & $h m g l 5^{\prime}$ crRNA & ATTTTGTCTATGATAGACAA \\
\hline & hmgl & hmgl 3' crRNA & CCAACGATTGCCAAAGGTCA \\
\hline \multicolumn{4}{|c|}{ RTqPCR Primers } \\
\hline & cyp $51 \mathrm{~A}$ & cyp51A RT F e1 & CTTCTTTGCGTGCAGAGA \\
\hline & cyp $51 A$ & cyp51A RT R e2 & GGGGTCGTCAATGGACTA \\
\hline & cyp $51 B$ & cyp51B RT F e1 & CTTTTTCGACTGCCGCGC \\
\hline & cyp $51 B$ & cyp51B RT R e2 & AGGCGTAGTGAGTGGAGA \\
\hline
\end{tabular}


of anhydrous pyridine (Sigma), $100 \mu \mathrm{L}$ of N,O- bis(trimethylsilyl)trifluoroacetamide (BSTFA)- 10\% trimethylsilyl (TMS) (Sigma), and two hours of heating at 80C. Gas chromatography-mass spectrometry (GC-MS) (Thermo 1300 GC coupled to a Thermo ISQ mass spectrometer; Thermo Scientific) was then used to analyze and identify TMSderivatized sterols. Known standards were referenced for fragmentation spectra and retention times. Sterol profiles for each isolate were then created using Xcalibur software (Thermo Scientific) to analyze GC-MS data.[16] Statistical analysis of both sterol profiles and total ergosterol per dry weight were performed using GraphPad Prism 7. In all cases, 6 independent biological replicates were measured and included in analysis, two-tailed unpaired t-test were performed in Prism 8 for Mac OS by GraphPad Software Inc. with significance set at 0.05 and degrees of freedom equal to 10.

\section{Assessment cyp51A and cyp51B Expression by RTqPCR}

Conidia from each strain were allowed to germinate overnight on an orbital shaker at $250 \mathrm{RPM}$ incubated at $37^{\circ} \mathrm{C}$ in Aspergillus minimal media. RNA was then extracted from mature hyphae following liquid nitrogen crush as previously described.[68] cDNA was synthesized using the RevertAid RT kit (Thermo Scientific). $A$. fumigatus cyp $51 A$ and cyp $51 B$ were amplified from cDNA by PCR using PCR master mix and SYBR per the manufacturer's instructions. Table 4-1 lists the gene specific primers used for PCR. Conditions used for PCR were as follows: AmpliTaq Gold activation at $95^{\circ} \mathrm{C}$ for 10 minutes, 40 cycles of denaturation at $95^{\circ} \mathrm{C}$ for 15 seconds and annealing/ extension at $60^{\circ} \mathrm{C}$. The dissociation curve and threshold cycle (CT) was determined using the CFX96 Real Time PCR system (Bio-Rad). Changes in gene expression among isolates were then calculated using the 2- $\Delta \Delta \mathrm{CT}$ method. All experiments were performed in triplicate from biological triplicates. As previously described, $\Delta \mathrm{CT}$ values were used to calculate the standard error.[68] Statistically analysis was performed using unpaired, two-tailed, students t-test in Prism 8 for Mac OS by GraphPad Software Inc. with significance set at 0.05 and degrees of freedom equal to 10.

\section{Results}

\section{Mutations in $\mathbf{h m g} 1$ Are Common Among Triazole-Resistant Clinical Isolates of $\boldsymbol{A}$. fumigatus}

Following our prior findings that the previously characterized mechanisms of triazole-resistance did not explain the high level of triazole resistance observed in this collection of clinical A. fumigatus isolates, we sought to interrogate the previously generated whole genome sequencing data to identify novel mechanisms of resistance. As the triazoles exert antifungal activity through the inhibition of biosynthesis of the predominant fungal membrane sterol, ergosterol, initial whole genome sequencing analysis targeted genes encoding proteins known to participate in ergosterol biosynthesis. In addition to the previously identified cyp5 $1 \mathrm{~A}$ mutations, mutations unique to triazoleresistant isolates were also observed in erg3B (Afu2g00320), erg3C (Afu8g01070), 
erg4B (Afu1g07140), erg5 (Afu1g03950), and erg6 (Afu4g03630). However, mutations occurred in these genes among only a small number of the clinical isolates (Table 4-2). Intriguingly, 11 of the 21 triazole-resistant clinical isolates $(52 \%)$ were found to possess mutations in the $h m g l$ gene resulting in peptide sequence changes not observed among the triazole-susceptible control isolates (Figure 4-1A). The A. fumigatus hmgl gene encodes a HMG-CoA reductase enzyme previously reported to be essential. HMG-CoA reductase catalyzes the first committed step in ergosterol biosynthesis and has previously been shown in other eukaryotic organisms to participate in the regulation of sterol biosynthesis through direct interactions between sterols and a conserved sterol sensingdomain.[85-88] Of particular interest, eight of the eleven isolates with $\mathrm{hmgl}$ mutations were found to have mutations affecting residues predicted to reside within the conserved sterol-sensing domain of Hmg1 (Figure 4-1B). We additionally accessed publicly available whole genome sequencing data for 11 triazole-resistant clinical isolates (https://www.ebi.ac.uk/arrayexpress/experiments/E-SYBR-1/samples/) and found 5 to have mutations in $h m g 1,2$ of which alter residues in the predicted sterol sensing domain (P309L and I412T). As mutations in $h m g l$ were observed in a large proportion of clinical triazole-resistant isolates, and hmgl mutations have previously been observed among laboratory isolates of $A$. fumigatus grown in the presence of triazole antifungals and the closely related agricultural sterol-demethylase inhibitors, the observed mutations in $\mathrm{hmgl}$ were selected for further interrogation. $[47,89]$

\section{Mutations in hmgl Confer Resistance to the Triazole Class of Antifungals}

To test the hypothesis that mutations in hmg1 directly contribute to clinical triazole resistance in A. fumigatus, three mutations encoding amino acid substitutions or in-frame codon deletions in different transmembrane regions of the predicted $\mathrm{hmgl}$ sterol-sensing domain (F262del, S305P, and I412S) were selected for characterization (Figure 4-1B). Each of these mutations and a wild-type control allele were directly introduced to the native $h m g l$ locus of the triazole-susceptible laboratory strain $a k u B^{\mathrm{KU} 80}$ using a novel Cas9-RNP editing technique incorporating a split hygromycin B resistance marker (Figure 4-2). Briefly, hmgl alleles including the open reading frame and approximately 500 downstream bases were amplified by PCR from DI15-98 (S305P), DI15-100 (I412S), DI15-105 (F262del), and $a k u B^{\mathrm{KU} 80}$ (wild-type control) using a 3' primer which introduced the terminal 80 bases with homology to the 3 ' end of the $h p h R$ hygromycin B resistance gene open reading frame. A partial hygromycin B resistance cassette, including the $g d p A$ promoter and a truncated $h p h R$ gene lacking the terminal 40 bases, was then amplified by PCR from the pUCGH plasmid using primers that introduced approximately 70 bases of homology with the downstream region of hmg1.[90] Protoplasts of $a k u B^{\mathrm{KU} 80}$ were then transformed with RNP complexes, consisting of Cas9 protein and crRNA-trRNA duplexes targeting sequences immediately upstream and and approximately 500 bases downstream of the open reading frame of $h m g 1$, and $2 \mu \mathrm{g}$ of each portion of the split marker repair template (a single $h m g l$ allele and the truncated hygromycin B resistance cassette), to produce $\mathrm{hmgl}$ mutant strains 
Table 4-2. Mutations in genes involved in ergosterol biosynthesis which are unique to triazole resistant isolates.

\begin{tabular}{|c|c|c|c|c|c|c|c|c|c|c|c|c|c|c|c|c|c|c|c|c|c|}
\hline Isolate & 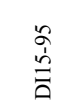 & $\begin{array}{l}\stackrel{\circ}{\circ} \\
\stackrel{\vec{b}}{\vec{n}}\end{array}$ & $\begin{array}{c}\hat{a} \\
\stackrel{\hat{\theta}}{\hat{\theta}}\end{array}$ & $\begin{array}{l}\text { a } \\
\stackrel{\hat{n}}{\overrightarrow{0}} \\
\end{array}$ & $\begin{array}{l}\stackrel{a}{a} \\
\stackrel{\vec{a}}{a}\end{array}$ & $\begin{array}{l}\frac{8}{1} \\
\frac{1}{0}\end{array}$ & 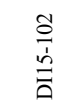 & $\begin{array}{l}\frac{8}{1} \\
\stackrel{1}{0} \\
\frac{\overrightarrow{0}}{0}\end{array}$ & $\begin{array}{l}\frac{2}{1} \\
\stackrel{1}{0} \\
\overline{0}\end{array}$ & $\begin{array}{l}\frac{8}{1} \\
\stackrel{\vec{n}}{\bar{B}}\end{array}$ & $\begin{array}{l}\stackrel{\hat{े}}{\vec{b}} \\
\overline{\overrightarrow{0}}\end{array}$ & $\begin{array}{l}\stackrel{\infty}{\circ} \\
\stackrel{1}{0} \\
\stackrel{\overline{0}}{0}\end{array}$ & $\begin{array}{l}\frac{8}{1} \\
\dot{1} \\
\overline{0}\end{array}$ & $\begin{array}{l}\stackrel{ }{\vec{n}} \\
\stackrel{\vec{\theta}}{\partial}\end{array}$ & $\begin{array}{l}\vec{\Xi} \\
\stackrel{\vec{n}}{0} \\
\bar{a}\end{array}$ & 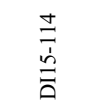 & $\begin{array}{l}\stackrel{n}{\vec{b}} \\
\stackrel{\vec{\theta}}{0}\end{array}$ & $\begin{array}{l}\stackrel{0}{7} \\
\stackrel{\vec{n}}{\vec{n}}\end{array}$ & $\begin{array}{l}\overline{7} \\
\stackrel{\bar{n}}{\bar{\theta}}\end{array}$ & $\begin{array}{l}\stackrel{\infty}{\vec{b}} \\
\stackrel{\vec{b}}{0}\end{array}$ & $\begin{array}{l}\stackrel{+}{7} \\
\stackrel{1}{0} \\
\overline{0}\end{array}$ \\
\hline $\operatorname{erg} 3 A$ & - & - & - & - & - & - & - & - & - & - & - & - & - & - & - & - & - & - & - & - & - \\
\hline $\operatorname{erg} 3 B$ & $\mathrm{H} 40 \mathrm{R}$ & - & - & - & - & $\mathrm{H} 40 \mathrm{R}$ & - & - & - & - & - & - & - & L88P & - & - & - & - & - & - & L88P \\
\hline erg $3 C$ & - & $\begin{array}{c}\text { P197fs, } \\
\text { R203S, } \\
\text { I205V, F252I, } \\
\text { Q310H }\end{array}$ & - & - & - & - & P317S & - & - & $\begin{array}{c}\text { P197fs, } \\
\text { R203S, } \\
\text { I205V, F252I, } \\
\text { Q310H }\end{array}$ & - & - & - & - & - & - & - & P317S & - & - & - \\
\hline $\operatorname{erg} 4 A$ & - & - & - & - & - & - & - & - & - & - & - & - & - & - & - & - & - & - & - & - & - \\
\hline $\operatorname{erg} 4 B$ & $\mathrm{D} 42 \mathrm{H}$ & - & $\begin{array}{c}\text { T90I, } \\
\text { H93N, } \\
\text { H97N }\end{array}$ & - & $\mathrm{H} 97 \mathrm{~N}$ & $\mathrm{D} 42 \mathrm{H}$ & - & - & $\begin{array}{l}\text { H97N, } \\
\text { A505S }\end{array}$ & - & - & $\mathrm{D} 42 \mathrm{H}$ & - & - & $\mathrm{D} 42 \mathrm{H}$ & - & $\mathrm{D} 42 \mathrm{H}$ & - & - & - & - \\
\hline erg 5 & - & K363R & F68L & - & - & - & - & - & - & K363R & - & - & - & - & - & - & - & $\mathrm{K} 363 \mathrm{R}$ & - & - & - \\
\hline erg6 & - & - & - & - & - & - & - & - & $\mathrm{F} 148 \mathrm{C}$ & - & - & - & - & - & F311del & M187L & - & A291T & L234I & - & - \\
\hline erg $25 A$ & - & - & - & - & - & - & - & - & - & - & - & - & - & - & - & - & - & - & - & - & - \\
\hline $\operatorname{erg} 25 B$ & - & - & - & - & - & - & - & - & - & - & - & - & - & - & - & - & - & - & - & - & - \\
\hline hmgl & $\mathrm{I} 412 \mathrm{~S}$ & $\begin{array}{l}\mathrm{G} 466 \mathrm{~V} \\
\mathrm{~S} 541 \mathrm{G}\end{array}$ & $\begin{array}{l}\text { S305P, } \\
\text { V995I }\end{array}$ & S305P & - & $\mathrm{I} 412 \mathrm{~S}$ & E105K & - & F262del & - & - & $\mathrm{Y} 250 \mathrm{H}$ & V995I & L413P & - & G307D & - & - & - & - & - \\
\hline
\end{tabular}




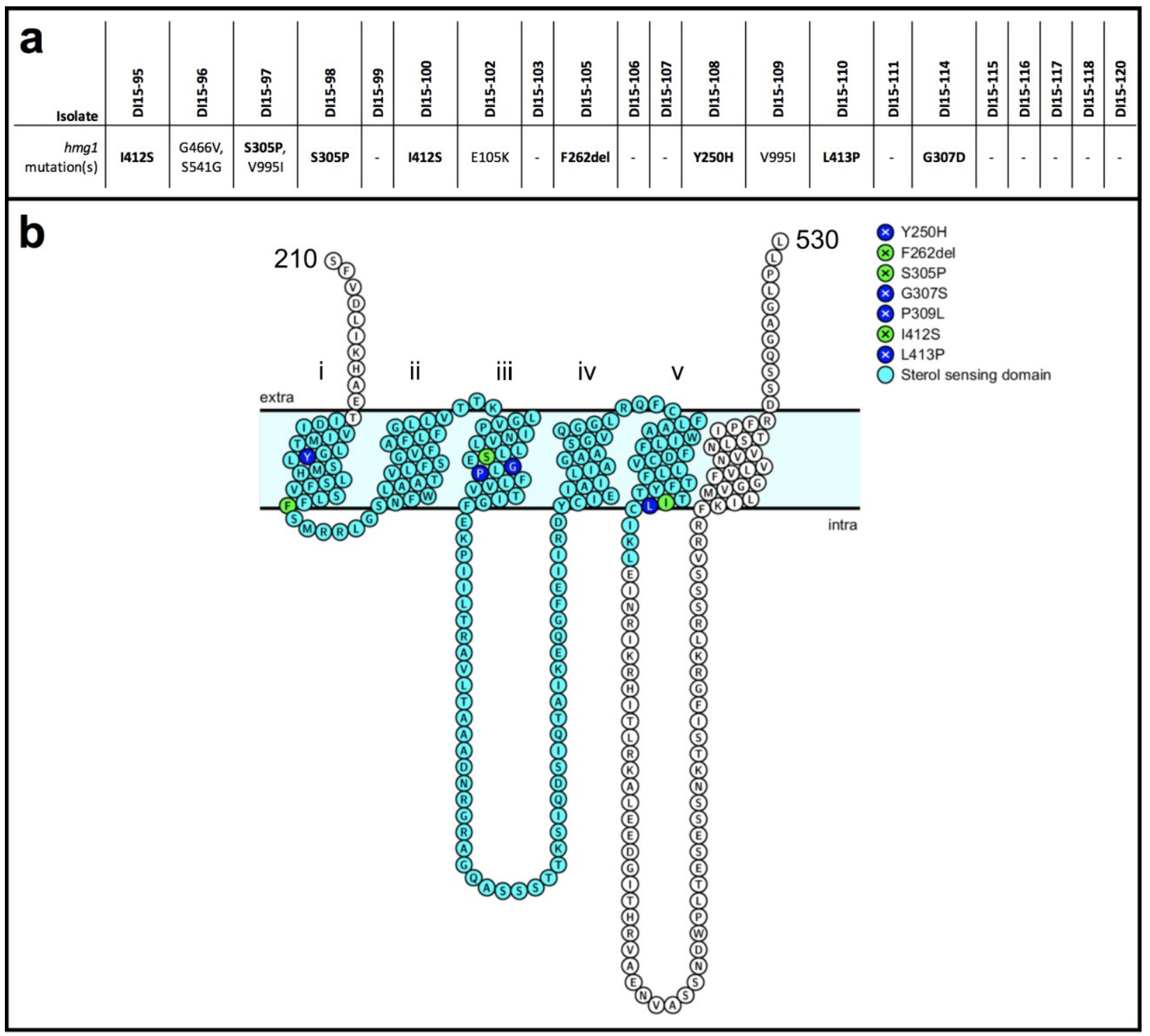

Figure 4-1. Mutations in hmgl observed in this collection of triazole-resistant clinical $A$. fumigatus isolates.

A) $h m g 1$ mutations observed in each clinical isolate relative to the Af293 reference genome, with mutations affecting residues predicted to reside within the conserved sterolsensing domain shown in bold. B) Schematic depicting the predicted transmembrane domains of A. fumigatus Hmg1. Residues in light blue comprise the predicted sterolsensing domain, residues in dark blue represent those affected by hmgl mutations observed in this collection of clinical isolates, residues shown in green represent those affected by hmgl mutations observed in this collection of clinical isolates and further characterized in this work. 


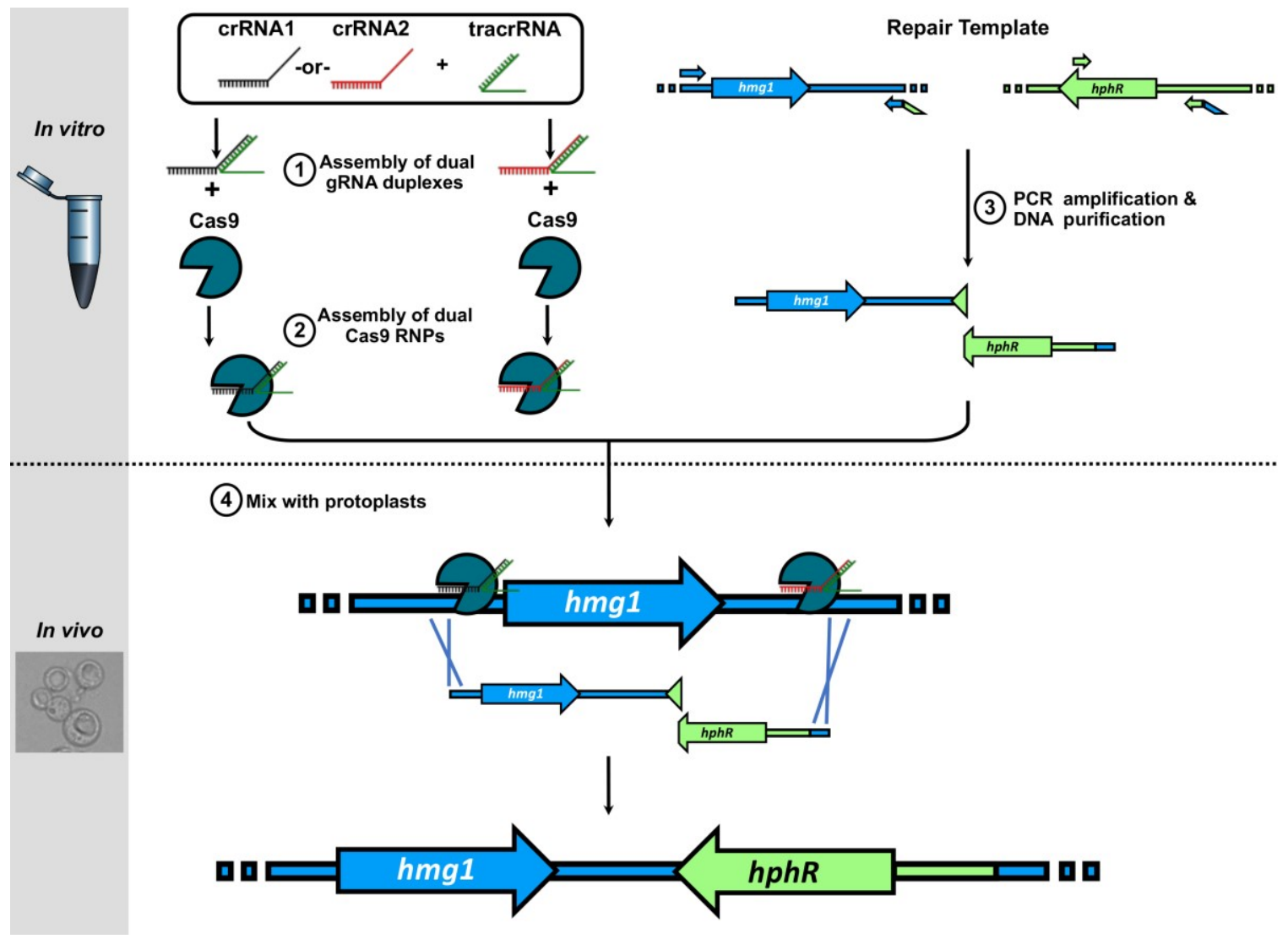

Figure 4-2. Schematic of Cas9 ribonucleoprotein (RNP) editing technique incorporating a split hygromycin B resistance marker for creation of hmgl mutant strains. 


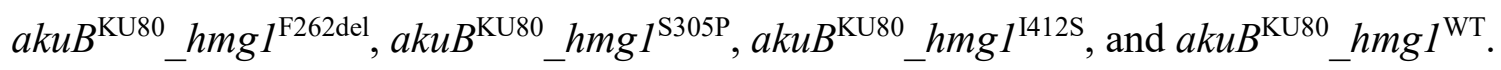

Antifungal susceptibility testing was subsequently performed for $a k u B^{\mathrm{KU} 80}$ and each of the derivative strains for voriconazole, isavuconazole, itraconazole, posaconazole and amphotericin B according to the Clinical Laboratory Standards Institute M38-A2 standards (Figure 4-3A). [69] The parental $a k u B^{\mathrm{KU} 80}$ strain was susceptible to all agents with MIC of $0.25,0.5,0.125,0.06$, and $0.5 \mathrm{mg} / \mathrm{L}$ for voriconazole, isavuconazole, itraconazole, posaconazole, and amphotericin B, respectively. The $a k u B^{\mathrm{KU} 80} h m g l^{\mathrm{WT}}$ control strain exhibited MIC exactly matching those of the parental strain. Conversely, all strains harboring a clinically derived mutation in $h m g l$ were found to exhibit a 4 to 8 -fold increase in MIC for all tested triazoles, exceeding the CLSI reported epidemiologic cutoff values for at least one agent in each mutant strain. $[73,82]$ Notably amphotericin B MIC were observed to decrease by two-fold in both the $a k u B^{\mathrm{KU} 80} h m g 1^{\mathrm{S} 305 \mathrm{P}}$ and $a k u B^{\mathrm{KU} 80} h m g l^{\mathrm{F} 262 \mathrm{del}}$ strains $(0.5$ versus $0.25 \mathrm{mg} / \mathrm{L})$, but not in $a k u B^{\mathrm{KU} 80} h m g 1^{1412 \mathrm{~S}}$.

In an effort to delineate the extent of $h m g l$-mutation-mediated triazole resistance within a clinical isolate from our collection, the mutant hmg1 allele in the pan-triazoleresistant clinical isolate DI15-105 was replaced with the wild-type $h m g l$ allele from $a k u B^{\mathrm{KU} 80}$ using the same Cas9-RNP-mediated gene editing described above. The resultant DI15-105_hmg ${ }^{\mathrm{WT}}$ strain exhibited complete restoration of triazole susceptibility with an eight-fold or greater decrease in MIC for each triazole agent (Figure $6 \mathrm{~b}$ ). Intriguingly, the amphotericin B MIC was observed to increase by two-fold in DI15-105_hmg $1^{\text {WT }}$ relative to DI15-105 (0.125 versus $\left.0.25 \mathrm{mg} / \mathrm{L}\right)$.

\section{Mutations in hmg1 Lead to Accumulation of Ergosterol Precursors}

As the sterol-sensing domain of HMG-CoA reductase has previously been shown to interact with ergosterol precursors and participate in the negative regulation of HMGCoA reductase activity in both human cells and the model fission yeast

Schizosaccharomyces pombe, we hypothesized that the mutations identified in the sterol sensing domain of A. fumigatus hmgl may be leading to dysregulation of the ergosterol biosynthesis pathway resulting in the observed triazole resistance (Figure 4-4A). [85-88] To test this hypothesis, comprehensive sterol profiling including assessment of the relative distribution of cell sterols and the measurement of total ergosterol content per dry weight was performed on $a k u B^{\mathrm{KU} 80}, a k u B^{\mathrm{KU} 80} h m g 1^{\mathrm{F} 262 \mathrm{del}}, a k u B^{\mathrm{KU} 80}{ }_{-} h m g 1^{\mathrm{S} 305 \mathrm{P}}$, $a k u B^{\mathrm{KU} 80}{ }_{-} m g 1^{1412 \mathrm{~S}}, a k u B^{\mathrm{KU} 80}{ }_{-} h m g 1^{\mathrm{WT}}$.

To assess the relative distribution of sterols among A. fumigatus harboring $h m g 1$ mutations, freshly harvested conidia stocks of each strain were grown in six biological replicates in RPMI media supplemented with $0.2 \%$ glucose and buffered with MOPS (pH 7.0) for 24 hours before cells were flash frozen with liquid nitrogen, dry weights were obtained, and nonsaponifiable lipids were extracted, derivatized, and analyzed by gaschromatography mass spectrometry as described previously.[16, 91] In agreement with the hypothesis that mutations in $\mathrm{hmgl}$ precipitate dysregulation of the ergosterol 


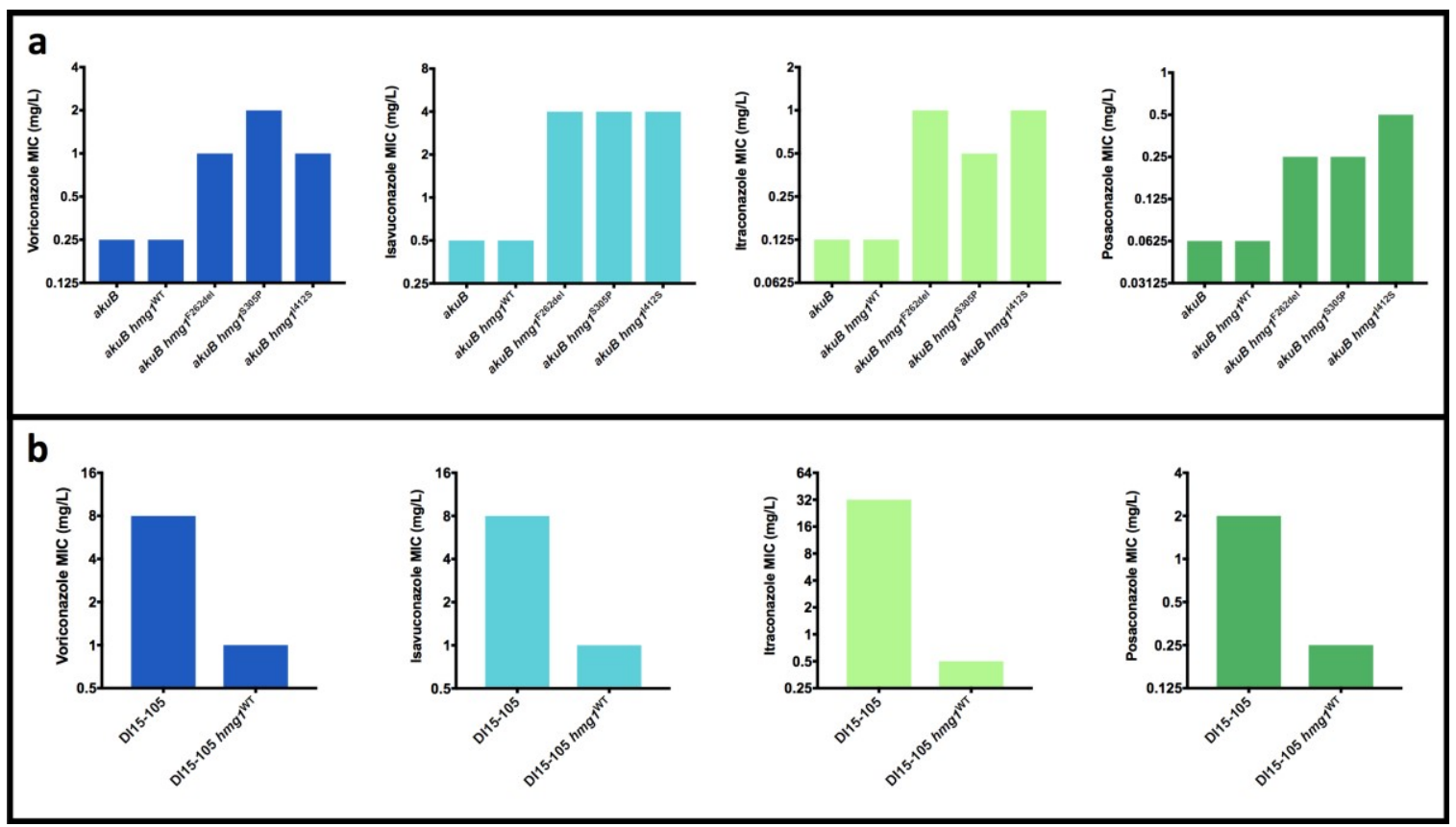

Figure 4-3. Impact of $\mathbf{h m g} \mathbf{l}$ mutations on antifungal susceptibility.

A) Triazole MIC for $a k u B^{\mathrm{KU} 80}$ and derivative mutant $h m g l$ strains B) Triazole MIC for DI15-105 and derivative $h m g l^{\text {WT }}$ strain. 


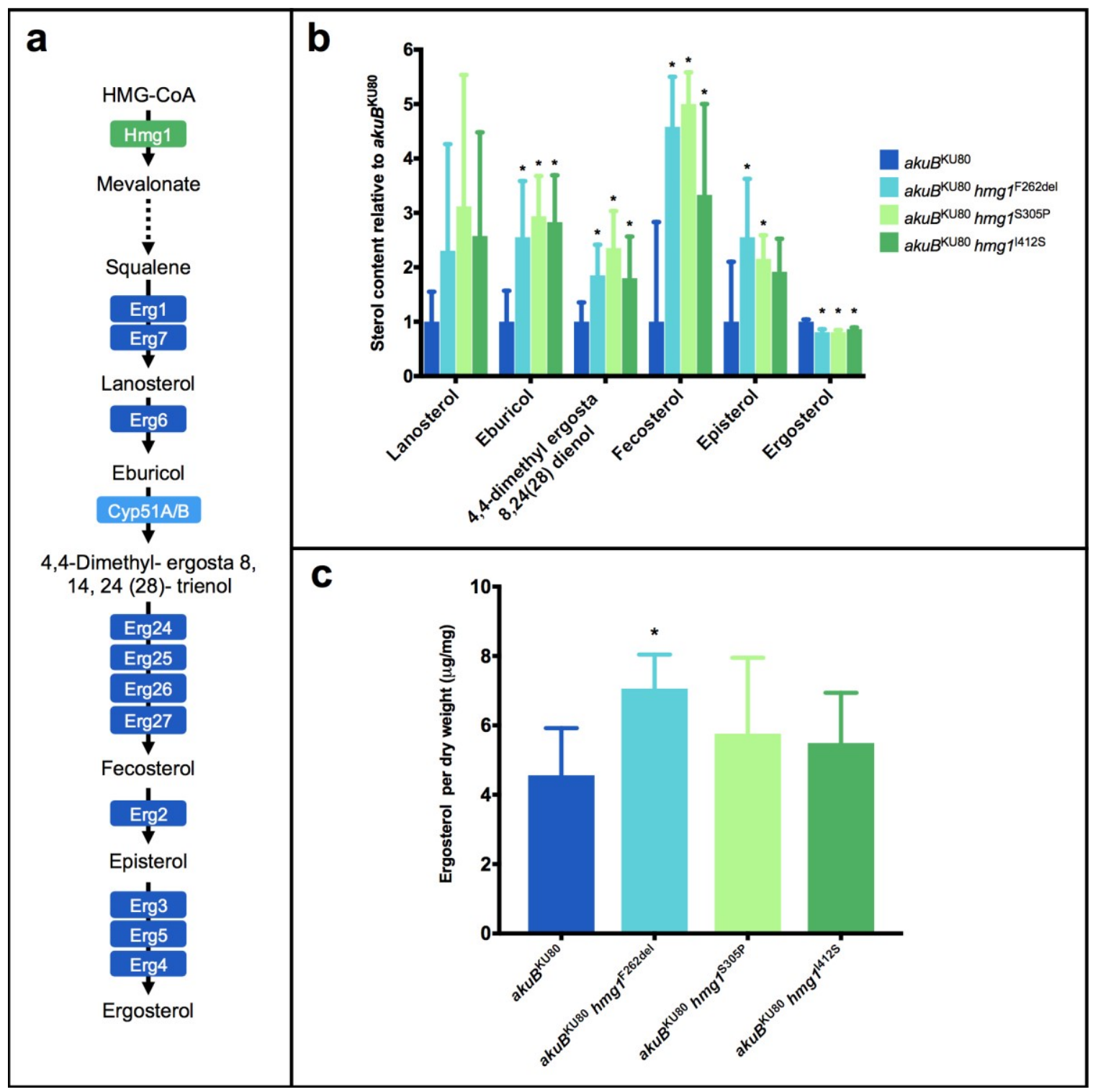

Figure 4-4. Relative distribution of sterols and total ergosterol content of $\mathbf{h m g l}$ mutant strains.

A) A. fumigatus ergosterol biosynthetic pathway B) Relative fold change of cellular sterols lanosterol, eburicol, 4,4-dimethyl ergosta 8,24(28) dienol, fecosterol, episterol, and ergosterol in $h m g l$ mutant strains as compared to the parental $a k u B^{\mathrm{KU} 80}$ strain $\mathbf{C}$ ) Total cellular ergosterol per dry weight for $h m g l$ mutant strains and the parental $a k u B^{\mathrm{KU} 80}$ strain. Comparisons with the susceptible parental isolate $a k u B^{\mathrm{KU} 80}$ with statistically significant $(\mathrm{p}<0.05)$ results are noted with an Asterix. 
biosynthetic pathway, the distribution of sterols was dramatically altered in all $\mathrm{hmgl}$ mutant strains relative to $a k u B^{\mathrm{KU} 80}$ (Figure 4-4B). Most notably, a significant decrease in the relative proportion of ergosterol, along with corresponding increases in the proportions of several ergosterol precursors downstream of Hmg1, including eburicol, 4,4-dimethyl ergosta 8,24(28) dienol, and fecosterol, were observed in all mutant $\mathrm{hmgl}$ strains. Additionally, the relative proportion of episterol was observed to be significantly elevated in $a k u B^{\mathrm{KU} 80} h m g 1^{\mathrm{F} 262 \mathrm{del}}$ and $a k u B^{\mathrm{KU} 80} h m g 1^{\mathrm{S} 305 \mathrm{P}}$. Importantly, no significant

differences in the relative distribution of sterols was observed between $a k u B^{\mathrm{KU} 80}$ and $a k u B^{\mathrm{KU} 80} h m g 1^{\mathrm{WT}}$ (not shown).

While the distribution of cellular sterols among A. fumigatus harboring hmgl mutations demonstrated a significant decrease in the relative proportion of cellular ergosterol, total ergosterol content per dry weight was not lower among any of the mutant $h m g l$ strains (Figure 4-4C). In fact, relative to the parental $a k u B^{\mathrm{KU} 80}$, $a k u B^{\mathrm{KU} 80} h m g 1^{\mathrm{F} 262 \mathrm{del}}$ was observed to exhibit a significant increase in total ergosterol (4.56 v. $7.06 \mu \mathrm{g}$ ergosterol/ $\mathrm{mg}$ dry weight; $\mathrm{p}<0.01$ ). Taken together, these findings demonstrate $h \mathrm{mgl}$ mutations precipitate a relative increase in ergosterol precursors downstream of Hmg1, while maintaining or in some cases even increasing cellular ergosterol, leading to significantly altered cellular sterol profiles among mutant $h m g l$ strains.

\section{Mutations in hmgl Do Not Lead to Increased Expression of Sterol-Demethylase Genes}

As our sterol profiling studies revealed significant accumulations of ergosterol precursors both upstream and downstream of sterol-demethylase, and we had previously observed that increased expression of either sterol-demethylase gene contributes to triazole resistance, we sought to investigate if the triazole resistance associated with mutations in $h m g l$ were at least in part mediated by altered expression of steroldemethylase genes. To accomplish this, conidia suspensions of $a k u B^{\mathrm{KU} 80}$, $a k u B^{\mathrm{KU} 80} h m g 1^{\mathrm{F} 262 \mathrm{del}}, a k u B^{\mathrm{KU} 80} h m g 1^{\mathrm{S} 305 \mathrm{P}}, a k u B^{\mathrm{KU} 80} h m g 1^{1412 \mathrm{~S}}$, and $a k u B^{\mathrm{KU} 80} h m g 1^{\mathrm{WT}}$ were grown in biological triplicate under the same conditions used for sterol profile analysis, and RNA was extracted from liquid nitrogen-pulverized samples, as previously described.[68] RTqPCR was then performed to assess expression of the steroldemethylase genes cyp5 $1 \mathrm{~A}$ and cyp51B among mutant $h m g l$ strains relative to the parental $a k u B^{\mathrm{KU} 80}$. No significant differences in expression of either sterol-demethylase gene was observed for any of the mutant $h m g l$ strains or the $a k u B^{\mathrm{KU}}{ }_{-} h m g l^{\mathrm{WT}}$ control strain (Figure 4-5). Demonstrating that while mutant $h m g l$ strains are observed to have relatively greater proportions of ergosterol precursors, expression levels of both cyp $51 \mathrm{~A}$ and cyp $51 B$ remain unchanged. Thus, resistance in these strains is not due to increased expression of these genes. 


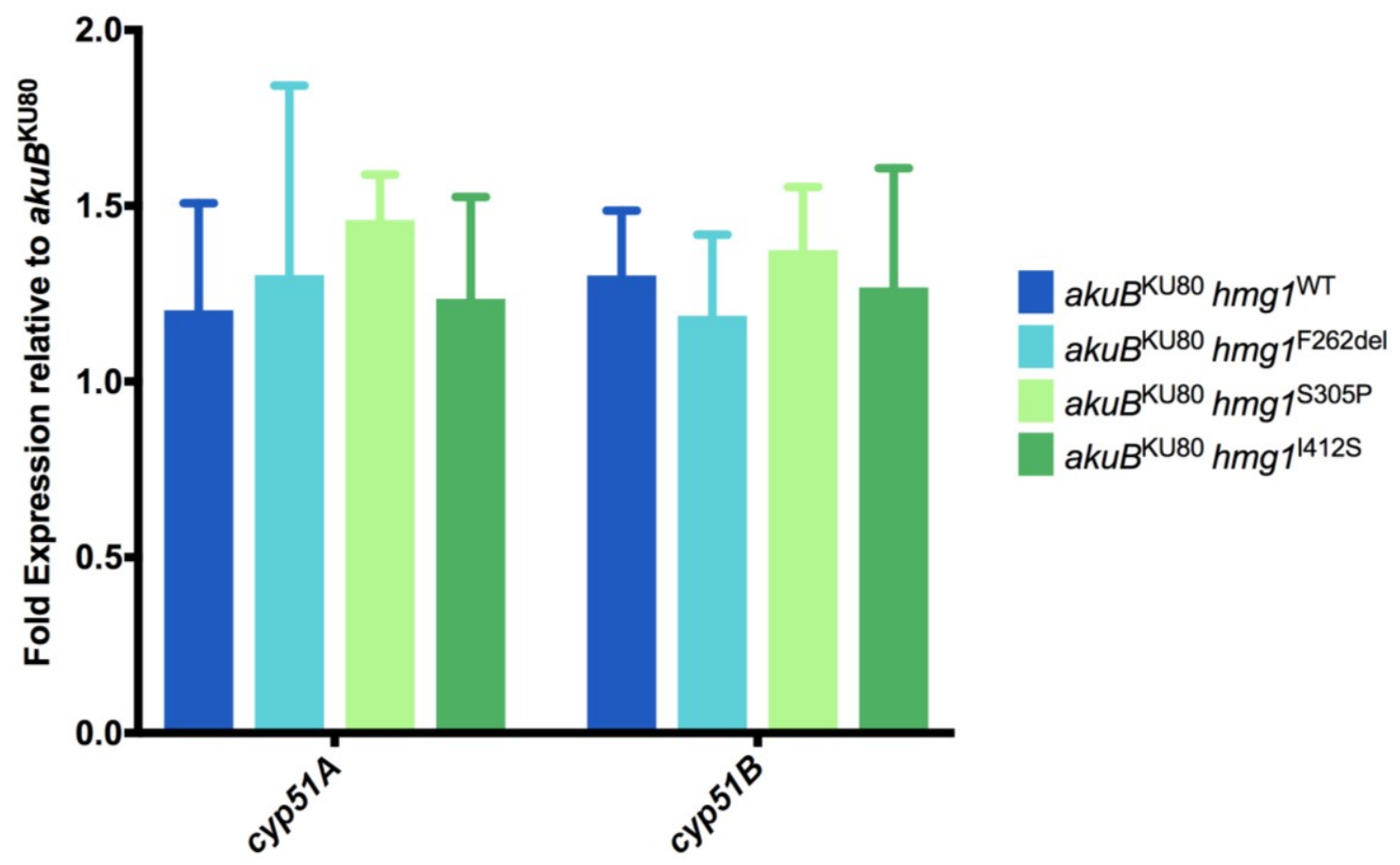

Figure 4-5. Expression of sterol-demethylase genes is not elevated among mutant hmg1 strains.

Fold expression of cyp $51 \mathrm{~A}$ and cyp $51 \mathrm{~B}$ in mutant $\mathrm{hmg} /$ strains relative to the parental $a k u B^{\mathrm{KU} 80}$ strain as determined by reverse transcription quantitative PCR. 


\section{Conclusions}

Here we describe for the first time, a novel and non-canonical mechanism of triazole antifungal resistance. Identified through whole genome sequencing, mutations in the HMG-CoA reductase encoding gene, $h m g l$, were found among the majority of triazole-resistant $A$. fumigatus clinical isolates in our collection. Of the observed mutations, most occurred in the conserved sterol sensing domain of $h m g l$, which has been previously demonstrated to participate in the regulation of sterol biosynthesis in other eukaryotic organisms including S. pombe.[85-88] When three of these clinicallyderived $h m g 1$ mutations were independently introduced into a well characterized triazolesusceptible strain of $A$. fumigatus using an optimized Cas9-mediated system to replace the native $h m g l$ locus, resistance to all clinically available anti-Aspergillus triazole antifungals greatly increased. To confirm that mutations in hmgl are a novel mechanism of clinical triazole resistance, we employed the same system to correct a $\mathrm{hmgl}$ mutation identified in a pan-triazole resistant clinical isolate of A. fumigatus and observed restoration of susceptibility to all triazoles.

As the sterol-sensing domain of HMG-CoA reductase has been shown to be essential to the regulation of sterol biosynthesis, we hypothesized that amino acid substitutions in this domain of $\mathrm{Hmg} 1$ may lead to impaired negative regulation of $\mathrm{Hmg} 1$ activity. [85-88] In an effort to test this hypothesis, we performed comprehensive sterol profiling on the mutant $\mathrm{hmgl}$ strains. This analysis identified significant accumulations of ergosterol precursors, including eburicol, 4,4-dimethyl ergosta 8,24(28) dienol, fecosterol, episterol, while total cellular ergosterol content was seen to be maintained, or in the case of the $h m g l^{\text {F262del }}$ mutation, significantly increased. However, this increase in ergosterol precursors did not result in a subsequent increase in sterol-demethylase expression levels in any of the mutant $h m g l$ strains. Thus, $h m g l$ mutation-mediated triazole resistance is not the result of increased sterol-demethylase gene expression.

Taken together, the results of this study identify mutations in the A. fumigatus HMG-CoA reductase gene, $h m g l$, as a novel and non-canonical genetic determinant of clinical triazole resistance, present among a large proportion of resistant clinical isolates. While the exact mechanism by which mutations in the sterol-sensing domain of $\mathrm{hmgl}$ impart clinical triazole resistance remains unknown at this time, it is tempting to speculate that the negative regulation of Hmg1 activity, which has been shown to be dependent on the sterol-sensing domain in other eukaryotic organisms, may be altered by residue substitutions in the sterol-sensing domain of A. fumigatus Hmg1. 


\section{CHAPTER 5. DISCUSSION}

Invasive aspergillosis is a leading cause of morbidity and mortality among immunocompromised populations and is predicted to cause more than 200,000 lifethreatening infections each year.[1, 4, 83, 84] Aspergillus fumigatus is the predominant pathogen isolated from patients with invasive aspergillosis, and accounts for more than $60 \%$ of all cases.[2] Only three classes antifungal agents are currently available for the treatment of invasive infections caused by Aspergillus fumigatus. Of these, the triazoles represent both therapeutic agents of choice, such as voriconazole and isavuconazole, as well as agents commonly used for salvage therapies such as itraconazole and posaconazole. As other antifungal agents such as the polyene amphotericin B and the echinocandins possess limitations in formulation, safety profile, or lack consistent fungicidal activity against $A$. fumigatus, the triazoles have proven to be essential to the treatment of invasive aspergillosis. Thus, the recent emergence of triazole-resistant $A$. fumigatus represents a critical threat to the current treatment of invasive aspergillosis.[10]

While considerable research has been performed to interrogate the mechanisms by which A. fumigatus becomes resistant to triazole antifungals, the overwhelming majority of resistance has been ascribed to three canonical paths that were originally described among pathogenic fungi decades ago; 1) mutations in sterol-demethylase genes, 2) overexpression of sterol-demethylase genes, or 3) overexpression of efflux pumpencoding genes. [25, 28, 34, 35, 42-44, 48, 66, 71, 79] Of these three paths, mutations in the A. fumigatus sterol demethylase gene cyp $51 \mathrm{~A}$ are the most commonly reported in the literature. However, the proportion of clinical triazole resistance explained by these canonical pathways remains poorly characterized. Additionally, resistant clinical isolates exhibiting none of these previously identified mechanisms have been reported.[25] This gap in the current understanding of the molecular mechanisms underpinning clinical triazole resistance in $A$. fumigatus is a significant barrier to the preservation of the utility of the triazoles and the design of novel therapeutic strategies to overcome triazoleresistant aspergillosis. The overall objective of this work was to overcome this barrier by performing a comprehensive characterization of the direct contributions of previously identified mechanisms of triazole resistance in a large collection of highly triazoleresistant clinical A. fumigatus isolates, and to identify novel mechanisms where unexplained clinical resistance was found.

In Chapter 2 we sought to test the hypothesis that the previously identified mechanisms of triazole resistance do not fully account for the degree of triazole resistance observed among clinical isolates of A. fumigatus. To accomplish this, we utilized a large collection of triazole-resistant clinical A. fumigatus isolates and whole genome sequencing, to identify all mutations in the sterol-demethylase genes cyp51A and cyp $51 B$, and well the previously identified sterol-demethylase cis- and trans-regulatory elements. While no mutations unique to resistant isolates were identified in cyp $51 B$, unique mutations in cyp $51 \mathrm{~A}$ were found in the vast majority of resistant isolates. However, correction of ten of these cyp51A mutations to the wild-type sequence only partially or fully restored clinical triazole susceptibility in two and one clinical isolate 
background, respectively. Additionally, RTqPCR revealed significant overexpression of cyp51A was observed in eight of the twenty-one triazole resistant isolates, five of which had mutations in either the promoter of cyp $51 A$ or a known transcriptional regulator of cyp51A (hapE). However, we subsequently demonstrated that the constitutive overexpression of either sterol-demethylase gene by as much as thirty-five-fold, had a limited impact on triazole susceptibility in a triazole-susceptible laboratory isolate. Next, we examined the expression of the previously characterized A. fumigatus triazole efflux pump $a b c C$ in our collection of isolates by RTqPCR. Fourteen of the twenty-one triazoleresistant clinical isolates were observed to have significantly increased expression of $a b c C$. However, the majority of these isolates only exhibited a small increase in $a b c C$ ( 2.4 to 4.1 -fold) while only three were found to have more marked increases in expression (6.5 to 33.2-fold), and constitutive overexpression of $a b c C$ (7.8-fold) in a triazole-susceptible laboratory strain yielded no change in triazole susceptibility. These findings serve to highlight the fact that even when considered collectively, the previously characterized mechanisms of resistance do not adequately explain the triazole resistance observed in this collection of A. fumigatus clinical isolates, and additional mechanisms yet to be identified are likely present in this collection.

Subsequently, in Chapter 3 we tested the hypothesis that the constitutive overexpression of efflux pump-encoding genes, other than $a b c C$, directly contributes to clinical triazole resistance in A. fumigatus. To accomplish this, we utilized the collection of clinical A. fumigatus isolates we had previously characterized and transcriptional profiling to identify a subset of $A$. fumigatus efflux pump-encoding genes with a high degree of homology to the well characterized C. albicans triazole efflux pump Cdr1, which were uniquely, constitutively overexpressed among triazole-resistant clinical isolates. We then identified $a b c A$ and atrI as the genes of greatest interest, and utilized Cas9-mediated genetic manipulations to delineate the direct contributions of these genes to triazole resistance. Unexpectedly, we found that deletion of these genes in triazoleresistant clinical isolates known to overexpress them, did not to result in meaningful changes in triazole susceptibility. However, it is worth noting that these results may have been limited by the presence of additional resistance mechanisms in these isolates, such as cyp $51 \mathrm{~A}$ mutations. While these findings could not definitively demonstrate a role for either $a b c A$ or atrI in clinical triazole resistance, they do serve to demonstrate the potential for additional efflux pump-encoding genes to participate in clinical triazole resistance.

Finally, in Chapter 4 we utilized our previously generated whole genome sequencing data to test the hypothesis that mutations in genes involved in the biosynthesis of ergosterol, other than sterol-demethylase, significantly contribute to clinical triazole resistance. Following careful examination of our whole genome sequencing data, we identified numerous genes of the ergosterol biosynthesis pathway $(\operatorname{erg} 3 B, \operatorname{erg} 3 C, \operatorname{erg} 4 B, \operatorname{erg} 5, \operatorname{erg} 6$, and $h m g 1)$ where mutations unique to triazole-resistant isolates were present. Of these, we found mutations in the HMG-CoA-reductase gene hmgl to be the most intriguing, as the majority of resistant clinical isolates in our collection were observed to have mutations in this gene, and previous studies in other eukaryotic organisms such as Schizosaccharomyces pombe have found $h m g l$ homologs 
to contain conserved sterol sensing domains involved in the regulation of the ergosterol biosynthesis pathway. Additionally, the majority of the identified hmgl mutations were found to reside in the predicted sterol-sensing domain. As these sterol-sensing domain mutations each fell within one of three predicted transmembrane domains, we selected one representative mutation from each transmembrane domain and utilized a novel Cas9mediated split marker transformation system to introduce these mutant $h m g l$ alleles into a triazole-susceptible laboratory strain of A. fumigatus. Surprisingly, introduction of each of the three mutant $\mathrm{hmgl}$ alleles to the susceptible stain were found to dramatically increase MICs for all clinically available triazole agents. Moreover, the complementary experiment correcting the F262del $\mathrm{hmgl}$ mutation in the $\mathrm{hmgl}$ allele of the pan-triazoleresistant isolate DI15-105 resulted in the restoration of clinical susceptibility to all triazoles. Thus, proving that mutations in $h m g l$ are a novel genetic determinant of clinical triazole resistance in A. fumigatus.

As the sterol-sensing domain of HMG-CoA-reductase enzymes has been shown to participate in the autoregulation of HMG-CoA-reductase activity, and subsequently the regulation of ergosterol biosynthesis, we then hypothesized that mutations in the sterolsensing domain of $\mathrm{hmgl}$ lead to dysregulation of ergosterol biosynthesis. To test this hypothesis, we performed compressive sterol profiling on the cellular extracts from each of our mutant $\mathrm{hmgl}$ strains and the parental strain with a wild-type $h m g l$ allele. In agreement with our hypothesis, sterol profiling revealed that the mutant hmgl strains indeed exhibited accumulation of ergosterol precursors downstream HMG-CoAreductase activity, while also maintaining, or in some cases increasing, the total cellular ergosterol content. This finding was particularly intriguing as it is suggestive of a potential association between increased $\mathrm{hmgl}$ activity and triazole resistance. Additionally, HMG-CoA is a validated target for pharmalogical inhibition, and numerous drugs which inhibit the human form of this enzyme are currently clinically available. Future studies are needed to further characterize the role of hmgl mutations in clinical triazole resistance, and to identify any potential therapeutic strategies for overcoming triazole resistance associated with these mutations.

While the findings of the studies described herein identify mutations in $h m g l$ as a novel genetic determinant of clinical triazole resistance, we have yet to definitively identify the molecular mechanism(s) by which these mutations contribute to the observed resistance. It is however tempting to speculate, based upon these data and previously published findings, that triazole resistance may be the result of two molecular mechanisms related to altered cell sterol composition. First, the accumulation of ergosterol precursors such as eburicol, episterol, and fecosterol, as observed in our sterol profiling experiments, may alter baseline cell membrane characteristics sufficiently to diminish the impact of sterol-demethylase inhibition. It has been postulated that a portion of the antifungal effect of triazoles is a consequence of acute changes in membrane characteristics such as fluidity and integrity upon inhibition of sterol-demethylase activity. [92] Accumulation of ergosterol precursors, particularly those downstream of the sterol-demethylase enzymes such as episterol and fecosterol, may augment the impact of the triazoles on membrane characteristics by either temporarily facilitating continued ergosterol biosynthesis or through the modification of baseline membrane characteristics. 
Additionally, it is possible that the accumulation of ergosterol precursors indirectly mediates triazole resistance. Previous studies have shown that the accumulation of sterols such as eburicol can precipitate activation of stress response pathways similar to those triggered by exposure to triazole antifungals. [93] Thus, the accumulation of ergosterol precursors in isolates with mutations in $\mathrm{hmgl}$ may lead to increases in the constitutive expression of drug resistance effectors, such as efflux pump-encoding genes, and prime cells to rapidly respond to triazole exposure. However, future studies will be needed to explore the potential contribution of these molecular mechanisms to $\mathrm{hmgl}$ mutationmediated clinical triazole resistance.

In conclusion, the findings of the work described herein serve to demonstrate that clinical triazole resistance in A. fumigatus is most likely the result of a complex amalgamation of resistance mechanisms, including both mechanisms currently known and yet to be identified. Furthermore, the paradigm of the molecular mechanisms of triazole resistance established in fungal pathogens such as Candida albicans, offers only an incomplete explanation for the triazole resistance observed in clinical isolates of $A$. fumigatus. The addition of $\mathrm{hmgl}$ mutations to the known genetic determinants of triazole resistance advances our understanding of the molecular mechanisms underpinning clinical triazole resistance, yet a substantial proportion of resistance remains to be explained. Future directions for this work include the characterization of compounded triazole resistance mechanisms, such as the combination of cpy51A and $h m g 1$ mutations, as well as the further characterization of efflux pump-encoding genes which are differentially expressed among triazole-resistant clinical isolates. Additionally, $A$. fumigatus Hmg1 must be investigated as a potential target of pharmacological inhibition and an opportunity to for the development of a new therapeutic strategy to overcome clinical triazole resistance in A. fumigatus. 


\section{LIST OF REFERENCES}

1. Brown, G.D., et al., Hidden killers: human fungal infections. Sci Transl Med, 2012. 4(165): p. 165rv13.

2. $\quad$ Steinbach, W.J., et al., Clinical epidemiology of 960 patients with invasive aspergillosis from the PATH Alliance registry. J Infect, 2012. 65(5): p. 453-64.

3. Brissaud, O., et al., Invasive fungal disease in PICU: epidemiology and risk factors. Ann Intensive Care, 2012. 2(1): p. 6.

4. Maertens, J.A., et al., Isavuconazole versus voriconazole for primary treatment of invasive mould disease caused by Aspergillus and other filamentous fungi (SECURE): a phase 3, randomised-controlled, non-inferiority trial. Lancet, 2016. 387(10020): p. 760-9.

5. van Paassen, J., et al., Emerging aspergillosis by azole-resistant Aspergillus fumigatus at an intensive care unit in the Netherlands, 2010 to 2013. Euro Surveill, 2016. 21(30).

6. Hurst, S.F., et al., Isolation of azole-resistant Aspergillus fumigatus from the environment in the south-eastern USA. J Antimicrob Chemother, 2017. 72(9): p. 2443-2446.

7. $\quad$ van der Linden, J.W., et al., Clinical implications of azole resistance in Aspergillus fumigatus, The Netherlands, 2007-2009. Emerg Infect Dis, 2011. 17(10): p. 1846-54.

8. Wiederhold, N.P., et al., First Detection of TR34 L98H and TR46 Y121F T289A Cyp51 Mutations in Aspergillus fumigatus Isolates in the United States. J Clin Microbiol, 2016. 54(1): p. 168-71.

9. $\quad$ Camps, S.M., et al., Discovery of a HapE mutation that causes azole resistance in Aspergillus fumigatus through whole genome sequencing and sexual crossing. PLoS One, 2012. 7(11): p. e50034.

10. Patterson, T.F., et al., Practice Guidelines for the Diagnosis and Management of Aspergillosis: 2016 Update by the Infectious Diseases Society of America. Clin Infect Dis, 2016. 63(4): p. e1-e60.

11. Cordonnier, C., M. Bresnik, and R. Ebrahimi, Liposomal amphotericin B (AmBisome) efficacy in confirmed invasive aspergillosis and other filamentous fungal infections in immunocompromised hosts: a pooled analysis. Mycoses, 2007. 50(3): p. 205-9.

12. Herbrecht, R., et al., Voriconazole versus amphotericin B for primary therapy of invasive aspergillosis. N Engl J Med, 2002. 347(6): p. 408-15.

13. Rybak, J.M., et al., Isavuconazole: Pharmacology, Pharmacodynamics, and Current Clinical Experience with a New Triazole Antifungal Agent.

Pharmacotherapy, 2015. 35(11): p. 1037-51.

14. Kelly, S.L., et al., Resistance to fluconazole and cross-resistance to amphotericin $B$ in Candida albicans from AIDS patients caused by defective sterol delta5,6desaturation. FEBS Lett, 1997. 400(1): p. 80-2.

15. Hargrove, T.Y., et al., Structure-Functional Characterization of Cytochrome P450 Sterol 14alpha-Demethylase (CYP51B) from Aspergillus fumigatus and 
Molecular Basis for the Development of Antifungal Drugs. J Biol Chem, 2015. 290(39): p. 23916-34.

16. Warrilow, A.G., et al., In Vitro Biochemical Study of CYP51-Mediated Azole Resistance in Aspergillus fumigatus. Antimicrob Agents Chemother, 2015. 59(12): p. 7771-8.

17. Leonardelli, F., et al., Aspergillus fumigatus Intrinsic Fluconazole Resistance Is Due to the Naturally Occurring T301I Substitution in Cyp51Ap. Antimicrob Agents Chemother, 2016. 60(9): p. 5420-6.

18. Pfaller, M.A., et al., In vitro activities of isavuconazole and comparator antifungal agents tested against a global collection of opportunistic yeasts and molds. J Clin Microbiol, 2013. 51(8): p. 2608-16.

19. Lepak, A.J., et al., Isavuconazole (BAL4815) pharmacodynamic target determination in an in vivo murine model of invasive pulmonary aspergillosis against wild-type and cyp51 mutant isolates of Aspergillus fumigatus. Antimicrob Agents Chemother, 2013. 57(12): p. 6284-9.

20. Lepak, A.J., et al., Posaconazole pharmacodynamic target determination against wild-type and Cyp51 mutant isolates of Aspergillus fumigatus in an in vivo model of invasive pulmonary aspergillosis. Antimicrob Agents Chemother, 2013. 57(1): p. 579-85.

21. Krishnan, S., E.K. Manavathu, and P.H. Chandrasekar, A comparative study of fungicidal activities of voriconazole and amphotericin $B$ against hyphae of Aspergillus fumigatus. J Antimicrob Chemother, 2005. 55(6): p. 914-20.

22. Meletiadis, J., et al., Differential fungicidal activities of amphotericin $B$ and voriconazole against Aspergillus species determined by microbroth methodology. Antimicrob Agents Chemother, 2007. 51(9): p. 3329-37.

23. Manavathu, E.K., et al., A comparative study of the post-antifungal effect (PAFE) of amphotericin B, triazoles and echinocandins on Aspergillus fumigatus and Candida albicans. J Antimicrob Chemother, 2004. 53(2): p. 386-9.

24. Whaley, S.G., et al., Azole Antifungal Resistance in Candida albicans and Emerging Non-albicans Candida Species. Front Microbiol, 2016. 7: p. 2173.

25. Fraczek, M.G., et al., The cdrlB efflux transporter is associated with non-cyp51amediated itraconazole resistance in Aspergillus fumigatus. J Antimicrob Chemother, 2013. 68(7): p. 1486-96.

26. Gsaller, F., et al., Sterol Biosynthesis and Azole Tolerance Is Governed by the Opposing Actions of SrbA and the CCAAT Binding Complex. PLoS Pathog, 2016. 12(7): p. e1005775.

27. Abdolrasouli, A., et al., Genomic Context of Azole Resistance Mutations in Aspergillus fumigatus Determined Using Whole-Genome Sequencing. MBio, 2015. 6(3): p. e00536.

28. Krishnan Natesan, S., et al., In vitro-in vivo correlation of voriconazole resistance due to G448S mutation (cyp51A gene) in Aspergillus fumigatus. Diagn Microbiol Infect Dis, 2012. 74(3): p. 272-7.

29. Seyedmousavi, S., et al., The role of azoles in the management of azole-resistant aspergillosis: from the bench to the bedside. Drug Resist Updat, 2014. 17(3): p. $37-50$. 
30. Camps, S.M., et al., Rapid induction of multiple resistance mechanisms in Aspergillus fumigatus during azole therapy: a case study and review of the literature. Antimicrob Agents Chemother, 2012. 56(1): p. 10-6.

31. Diaz-Guerra, T.M., et al., A point mutation in the 14alpha-sterol demethylase gene cyp 51 A contributes to itraconazole resistance in Aspergillus fumigatus. Antimicrob Agents Chemother, 2003. 47(3): p. 1120-4.

32. Lescar, J., et al., Aspergillus fumigatus harbouring the sole Y121F mutation shows decreased susceptibility to voriconazole but maintained susceptibility to itraconazole and posaconazole. J Antimicrob Chemother, 2014. 69(12): p. 32447.

33. Mellado, E., et al., A new Aspergillus fumigatus resistance mechanism conferring in vitro cross-resistance to azole antifungals involves a combination of cyp $51 \mathrm{~A}$ alterations. Antimicrob Agents Chemother, 2007. 51(6): p. 1897-904.

34. Snelders, E., et al., Genotype-phenotype complexity of the TR46/Y121F/T289A cyp51A azole resistance mechanism in Aspergillus fumigatus. Fungal Genet Biol, 2015. 82: p. 129-35.

35. Snelders, E., et al., The structure-function relationship of the Aspergillus fumigatuscyp 51 A L98H conversion by site-directed mutagenesis: the mechanism of L98H azole resistance. Fungal Genet Biol, 2011. 48(11): p. 1062-70.

36. Mellado, E., et al., Substitutions at methionine 220 in the 14alpha-sterol demethylase (Cyp51A) of Aspergillus fumigatus are responsible for resistance in vitro to azole antifungal drugs. Antimicrob Agents Chemother, 2004. 48(7): p. 2747-50.

37. Vermeulen, E., et al., Azole-resistant Aspergillus fumigatus due to TR46/Y121F/T289A mutation emerging in Belgium, July 2012. Euro Surveill, 2012. 17(48).

38. Brillowska-Dabrowska, A., et al., Examination of cyp51A and cyp51B expression level of the first Polish azole resistant clinical Aspergillus fumigatus isolate. Acta Biochim Pol, 2015. 62(4): p. 837-9.

39. Buied, A., et al., High-level expression of cyp51B in azole-resistant clinical Aspergillus fumigatus isolates. J Antimicrob Chemother, 2013. 68(3): p. 512-4.

40. Alvarez-Moreno, C., et al., Azole-resistant Aspergillus fumigatus harboring TR34/L98H, TR46/Y121F/T289A and TR53 mutations related to flower fields in Colombia. Sci Rep, 2017. 7: p. 45631.

41. Nascimento, A.M., et al., Multiple resistance mechanisms among Aspergillus fumigatus mutants with high-level resistance to itraconazole. Antimicrob Agents Chemother, 2003. 47(5): p. 1719-26.

42. Bowyer, P., et al., Identification of novel genes conferring altered azole susceptibility in Aspergillus fumigatus. FEMS Microbiol Lett, 2012. 332(1): p. 10-9.

43. Meneau, I., A.T. Coste, and D. Sanglard, Identification of Aspergillus fumigatus multidrug transporter genes and their potential involvement in antifungal resistance. Med Mycol, 2016. 54(6): p. 616-27.

44. Paul, S., D. Diekema, and W.S. Moye-Rowley, Contributions of Aspergillus fumigatus ATP-binding cassette transporter proteins to drug resistance and virulence. Eukaryot Cell, 2013. 12(12): p. 1619-28. 
45. Paul, S. and W.S. Moye-Rowley, Functional analysis of an ATP-binding cassette transporter protein from Aspergillus fumigatus by heterologous expression in Saccharomyces cerevisiae. Fungal Genet Biol, 2013. 57: p. 85-91.

46. Hagiwara, D., et al., A Novel Zn2-Cys6 Transcription Factor AtrR Plays a Key Role in an Azole Resistance Mechanism of Aspergillus fumigatus by Coregulating cyp51A and cdr1B Expressions. PLoS Pathog, 2017. 13(1): p. e1006096.

47. Losada, L., et al., Genetic Analysis Using an Isogenic Mating Pair of Aspergillus fumigatus Identifies Azole Resistance Genes and Lack of MAT Locus's Role in Virulence. PLoS Pathog, 2015. 11(4): p. e1004834.

48. Wei, X., et al., Screening and Characterization of a Non-cyp51A Mutation in an Aspergillus fumigatus cox10 Strain Conferring Azole Resistance. Antimicrob Agents Chemother, 2017. 61(1).

49. Verweij, P.E., et al., Azole resistance surveillance in Aspergillus fumigatus: beneficial or biased? J Antimicrob Chemother, 2016. 71(8): p. 2079-82.

50. Chen, Y., et al., Epidemiology and Molecular Characterizations of Azole Resistance in Clinical and Environmental Aspergillus fumigatus Isolates from China. Antimicrob Agents Chemother, 2016. 60(10): p. 5878-84.

51. Snelders, E., et al., Emergence of azole resistance in Aspergillus fumigatus and spread of a single resistance mechanism. PLoS Med, 2008. 5(11): p. e219.

52. Steinmann, J., et al., Emergence of azole-resistant invasive aspergillosis in HSCT recipients in Germany. J Antimicrob Chemother, 2015. 70(5): p. 1522-6.

53. Tangwattanachuleeporn, M., et al., Prevalence of azole-resistant Aspergillus fumigatus in the environment of Thailand. Med Mycol, 2017. 55(4): p. 429-435.

54. Ashu, E.E., et al., Global Population Genetic Analysis of Aspergillus fumigatus. mSphere, 2017. 2(1).

55. Lestrade, P.P., et al., Diagnosis and management of aspergillosis in the Netherlands: a national survey. Mycoses, 2016. 59(2): p. 101-7.

56. Vazquez, J.A. and E.K. Manavathu, Molecular Characterization of a Voriconazole-Resistant, Posaconazole-Susceptible Aspergillus fumigatus Isolate in a Lung Transplant Recipient in the United States. Antimicrob Agents Chemother, 2016. 60(2): p. 1129-33.

57. Pham, C.D., et al., Passive surveillance for azole-resistant Aspergillus fumigatus, United States, 2011-2013. Emerg Infect Dis, 2014. 20(9): p. 1498-503.

58. Berger, S., et al., Azole Resistance in Aspergillus fumigatus: A Consequence of Antifungal Use in Agriculture? Front Microbiol, 2017. 8: p. 1024.

59. Fuhren, J., et al., High prevalence of azole resistance in Aspergillus fumigatus isolates from high-risk patients. J Antimicrob Chemother, 2015. 70(10): p. 28948.

60. Koehler, P., et al., Epidemiology of invasive aspergillosis and azole resistance in patients with acute leukaemia: the SEPIA Study. Int J Antimicrob Agents, 2017. 49(2): p. 218-223.

61. Verweij, P.E., et al., International expert opinion on the management of infection caused by azole-resistant Aspergillus fumigatus. Drug Resist Updat, 2015. 21-22: p. 30-40. 
62. Lavergne, R.A., et al., Home Environment as a Source of Life-Threatening AzoleResistant Aspergillus fumigatus in Immunocompromised Patients. Clin Infect Dis, 2017. 64(1): p. 76-78.

63. Lien, M.Y., et al., Epidemiology and risk factors for invasive fungal infections during induction chemotherapy for newly diagnosed acute myeloid leukemia: A retrospective cohort study. PLoS One, 2018. 13(6): p. e0197851.

64. Parker, J.E., et al., Resistance to antifungals that target CYP51. J Chem Biol, 2014. 7(4): p. 143-61.

65. Ballard, S.A., et al., Interaction of microsomal cytochrome P-450 isolated from Aspergillus fumigatus with fluconazole and itraconazole. J Med Vet Mycol, 1990. 28(4): p. 327-34.

66. Paul, S., D. Diekema, and W.S. Moye-Rowley, Contributions of both ATPBinding Cassette Transporter and Cyp51A Proteins Are Essential for Azole Resistance in Aspergillus fumigatus. Antimicrob Agents Chemother, 2017. 61(5).

67. Al Abdallah, Q., W. Ge, and J.R. Fortwendel, A Simple and Universal System for Gene Manipulation in Aspergillus fumigatus: In Vitro-Assembled Cas9-Guide RNA Ribonucleoproteins Coupled with Microhomology Repair Templates. mSphere, 2017. 2(6).

68. Fortwendel, J.R., et al., Transcriptional regulation of chitin synthases by calcineurin controls paradoxical growth of Aspergillus fumigatus in response to caspofungin. Antimicrob Agents Chemother, 2010. 54(4): p. 1555-63.

69. CLSI, Reference Method for Broth Dilution Antifungal Susceptibility Testing of Filamentous Fungi; Approved Standard-Second Edition. 2008: CLSI Document M38-A2.

70. Lestrade, P.P.A., et al., Triazole resistance in Aspergillus fumigatus: recent insights and challenges for patient management. Clin Microbiol Infect, 2018.

71. Umeyama, T., et al., CRISPR/Cas9 Genome Editing To Demonstrate the Contribution of Cyp51A Gly138Ser to Azole Resistance in Aspergillus fumigatus. Antimicrob Agents Chemother, 2018. 62(9).

72. Yelton, M.M., J.E. Hamer, and W.E. Timberlake, Transformation of Aspergillus nidulans by using a trpC plasmid. Proc Natl Acad Sci U S A, 1984. 81(5): p. 1470-4.

73. Pfaller, M.A., et al., Wild-type MIC distribution and epidemiological cutoff values for Aspergillus fumigatus and three triazoles as determined by the Clinical and Laboratory Standards Institute broth microdilution methods. J Clin Microbiol, 2009. 47(10): p. 3142-6.

74. Paul, S., J.S. Klutts, and W.S. Moye-Rowley, Analysis of promoter function in Aspergillus fumigatus. Eukaryot Cell, 2012. 11(9): p. 1167-77.

75. Weidner, G., et al., Development of a homologous transformation system for the human pathogenic fungus Aspergillus fumigatus based on the pyrG gene encoding orotidine 5'-monophosphate decarboxylase. Curr Genet, 1998. 33(5): p. 378-85.

76. Kano, R., et al., In vitro resistance of Aspergillus fumigatus to azole farm fungicide. J Infect Chemother, 2016. 22(3): p. 133-6.

77. Le Pape, P., et al., Multiple Fungicide-Driven Alterations in Azole-Resistant Aspergillus fumigatus, Colombia, 2015. Emerg Infect Dis, 2016. 22(1): p. 156-7. 
78. Rocchi, S., et al., Azole-resistant Aspergillus fumigatus isolate with the TR34/L98H mutation in both a fungicide-sprayed field and the lung of a hematopoietic stem cell transplant recipient with invasive aspergillosis. J Clin Microbiol, 2014. 52(5): p. 1724-6.

79. Rybak, J.M., J.R. Fortwendel, and P.D. Rogers, Emerging threat of triazoleresistant Aspergillus fumigatus. J Antimicrob Chemother, 2018.

80. Liu, T.T., et al., Genome-wide expression and location analyses of the Candida albicans Taclp regulon. Eukaryot Cell, 2007. 6(11): p. 2122-38.

81. Sanglard, D., et al., Susceptibilities of Candida albicans multidrug transporter mutants to various antifungal agents and other metabolic inhibitors. Antimicrob Agents Chemother, 1996. 40(10): p. 2300-5.

82. Espinel-Ingroff, A., et al., Multicenter study of isavuconazole MIC distributions and epidemiological cutoff values for Aspergillus spp. for the CLSI M38-A2 broth microdilution method. Antimicrob Agents Chemother, 2013. 57(8): p. 3823-8.

83. Blot, S., et al., The economic impact of invasive aspergillosis in intensive care unit patients. Int J Infect Dis, 2010. 14(6): p. e536-7.

84. Kim, A., D.P. Nicolau, and J.L. Kuti, Hospital costs and outcomes among intravenous antifungal therapies for patients with invasive aspergillosis in the United States. Mycoses, 2011. 54(5): p. e301-12.

85. Theesfeld, C.L., et al., The sterol-sensing domain (SSD) directly mediates signalregulated endoplasmic reticulum-associated degradation (ERAD) of 3-hydroxy-3methylglutaryl (HMG)-CoA reductase isozyme Hmg2. J Biol Chem, 2011. 286(30): p. 26298-307.

86. Xu, L. and R.D. Simoni, The inhibition of degradation of 3-hydroxy-3methylglutaryl coenzyme A (HMG-CoA) reductase by sterol regulatory element binding protein cleavage-activating protein requires four phenylalanine residues in span 6 of HMG-CoA reductase transmembrane domain. Arch Biochem Biophys, 2003. 414(2): p. 232-43.

87. Yasmin, S., et al., Mevalonate governs interdependency of ergosterol and siderophore biosyntheses in the fungal pathogen Aspergillus fumigatus. Proc Natl Acad Sci U S A, 2012. 109(8): p. E497-504.

88. Burg, J.S., et al., Insig regulates $H M G-C o A$ reductase by controlling enzyme phosphorylation in fission yeast. Cell Metab, 2008. 8(6): p. 522-31.

89. Zhang, J., et al., Evolution of cross-resistance to medical triazoles in Aspergillus fumigatus through selection pressure of environmental fungicides. Proc Biol Sci, 2017. 284(1863).

90. Langfelder, K., et al., Differential expression of the Aspergillus fumigatus pksP gene detected in vitro and in vivo with green fluorescent protein. Infect Immun, 2001. 69(10): p. 6411-8.

91. Venkateswarlu, K. and S.L. Kelly, Biochemical characterisation of ketoconazole inhibitory action on Aspergillus fumigatus. FEMS Immunol Med Microbiol, 1996. 16(1): p. 11-20.

92. Abe, F., K. Usui, and T. Hiraki, Fluconazole modulates membrane rigidity, heterogeneity, and water penetration into the plasma membrane in Saccharomyces cerevisiae. Biochemistry, 2009. 48(36): p. 8494-504. 
93. $\mathrm{Hu}, \mathrm{C}$., et al., Abnormal Ergosterol Biosynthesis Activates Transcriptional Responses to Antifungal Azoles. Front Microbiol, 2018. 9: p. 9. 


\section{VITA}

Jeffrey M. Rybak, son of Michael J. Rybak and Stephanie A. Rybak, was born in Royal Oak, Michigan in 1988. After graduating from Avondale High School in 2006, he moved to Detroit, Michigan to pursue college education at Wayne State University as part of the HealthPro Start Pre-Pharmacy program. In 2008, he was accepted to the Doctor of Pharmacy program at the Eugene Applebaum College of Pharmacy and Allied Health Sciences at Wayne State University. After receiving his Pharm.D. in 2012, he completed both a post-graduate year one Pharmacy Practice residency and a postgraduate year two Infectious Diseases Pharmacy residency at the University of Kentucky HealthCare in Lexington, Kentucky. He then moved to Memphis, Tennessee in 2014, to enter the Microbiology, Immunology, and Biochemistry track of the Integrated Biomedical Sciences program at the University of Tennessee College of Graduate Health Sciences. He expects to complete his doctoral degree by May of 2019. 